\title{
Medicinal Plants for the Treatment of Local Tissue Damage Induced by Snake Venoms: An Overview from Traditional Use to Pharmacological Evidence
}

\author{
Juliana Félix-Silva, ${ }^{1}$ Arnóbio Antônio Silva-Junior, ${ }^{1}$ \\ Silvana Maria Zucolotto, ${ }^{2}$ and Matheus de Freitas Fernandes-Pedrosa ${ }^{1}$ \\ ${ }^{1}$ Laboratório de Tecnologia \& Biotecnologia Farmacêutica (TecBioFar), Faculdade de Farmácia, \\ Universidade Federal do Rio Grande do Norte (UFRN), Natal, RN, Brazil \\ ${ }^{2}$ Grupo de Pesquisa em Produtos Naturais Bioativos (PNBio), Laboratório de Farmacognosia, Faculdade de Farmácia, \\ Universidade Federal do Rio Grande do Norte (UFRN), Natal, RN, Brazil
}

Correspondence should be addressed to Matheus de Freitas Fernandes-Pedrosa; mpedrosa31@uol.com.br

Received 23 February 2017; Accepted 9 July 2017; Published 21 August 2017

Academic Editor: Rainer W. Bussmann

Copyright (C) 2017 Juliana Félix-Silva et al. This is an open access article distributed under the Creative Commons Attribution License, which permits unrestricted use, distribution, and reproduction in any medium, provided the original work is properly cited.

\begin{abstract}
Snakebites are a serious problem in public health due to their high morbimortality. Most of snake venoms produce intense local tissue damage, which could lead to temporary or permanent disability in victims. The available specific treatment is the antivenom serum therapy, whose effectiveness is reduced against these effects. Thus, the search for complementary alternatives for snakebite treatment is relevant. There are several reports of the popular use of medicinal plants against snakebites worldwide. In recent years, many studies have been published giving pharmacological evidence of benefits of several vegetal species against local effects induced by a broad range of snake venoms, including inhibitory potential against hyaluronidase, phospholipase, proteolytic, hemorrhagic, myotoxic, and edematogenic activities. In this context, this review aimed to provide an updated overview of medicinal plants used popularly as antiophidic agents and discuss the main species with pharmacological studies supporting the uses, with emphasis on plants inhibiting local effects of snake envenomation. The present review provides an updated scenario and insights into future research aiming at validation of medicinal plants as antiophidic agents and strengthens the potentiality of ethnopharmacology as a tool for design of potent inhibitors and/or development of herbal medicines against venom toxins, especially local tissue damage.
\end{abstract}

\section{Introduction}

Snakebites are a serious public health problem in many regions around the world, particularly in Africa, Asia, Latin America, and parts of Oceania [1]. Conservative data indicate that, worldwide, there are between 1.2 and 5.5 million snakebites every year, leading to 25,000 to 125,000 deaths [2]. Despite its significant impact on human health, this condition remains largely neglected by national and international health authorities, funding agencies, pharmaceutical companies, patients' organizations, and health advocacy groups [1]. Thus, snake envenomation is included since 2009 in World Health Organization (WHO) list of Neglected Tropical Diseases (NTDs) [3]. Envenoming and deaths resulting from snakebites are a particularly important public health problem in the rural tropics. Populations in these regions experience high morbidity and mortality because of poor access to health services, which are often suboptimal, as well as other NTDs, which are associated with poverty $[3,4]$.

Snakes with major clinical importance belong to the families Elapidae (African and Asian cobras, Asian kraits, African mambas, American coral snakes, Australian and New Guinean venomous snakes, and sea snakes) and Viperidae (Old World vipers, American rattlesnakes and pit vipers, and Asian pit vipers) [5]. After production, snake venom is injected in the victim via tubular or channeled fangs [6]. Biochemically, venoms are complex mixtures of pharmacologically active proteins and polypeptides, acting in concert 
to help in immobilizing the prey [7]. The most common toxins in snake venoms are snake venom metalloproteinases (SVMPs), phospholipases $\mathrm{A}_{2}\left(\mathrm{PLA}_{2} \mathrm{~s}\right)$, snake venom serine proteinases (SVSPs), acetylcholinesterase (AChE), L-amino acid oxidases (LAAOs), nucleotidases, and snake venom hyaluronidases (SVHs) [7].

Biological properties of snake venom components are peculiar to each species, but in general, the main clinical effects of snake envenomation are immediate and prominent local tissue damage (including myonecrosis, dermonecrosis, hemorrhage, and edema), coagulation disorders (consumption coagulopathy and spontaneous systemic bleeding), cardiovascular alterations (hypotension, hypovolemic shock, and myocardial damage), renal alterations (which could evolve into acute kidney injure), neurotoxic action (descending paralysis, progressing from ptosis and external ophthalmoplegia to bulbar, respiratory muscle, and total flaccid paralysis), generalized rhabdomyolysis with myoglobinuria, and intravascular haemolysis $[5,8]$.

The only available specific treatment is the antivenom serum therapy, which consists of a pool of neutralizing immunoglobulins, or immunoglobulin fragments, purified from the plasma of animals hyperimmunized against snake venoms or specific toxins. Its effectiveness consists in its ability to provide to the patient antibodies with a high affinity to snake venom, aiming to eliminate the toxins responsible for toxicity of the envenoming, mitigating the progress of toxic effects induced by snake venom components [9]. However, the antivenom has some limitations, such as poor ability to treat local effects, risk of immunological reactions, high cost, and difficult access in some regions [8-10]. If antivenom administration is initiated rapidly after envenomation, neutralization of systemic effects is usually achieved successfully; however, neutralization of local tissue damage is more difficult [8]. Furthermore, the availability and accessibility of antivenoms is limited in many regions, such as Sub-Saharan Africa, Asia, and, to a lesser extent, Latin America, which could aggravate even more this picture [1]. Thus, this inability to treat local effects, as well as the increased time between accident and treatment, is the main reason for the temporary or permanent disability observed in many victims, which can lead to serious social, economic, and health negative impacts, given that most victims live in rural areas [3].

In this context, the search for complementary therapies to treat snakebites is relevant and medicinal plants could be highlighted as a rich source of natural inhibitors and pharmacologically active compounds $[6,11-13]$. There are several reports of the popular use of medicinal plants against snakebites around the world, especially in tropical and subtropical regions such as Asia, Africa, and South America $[14,15]$. The rural and tribal people living in remote areas greatly depend on folk medicines for the treatment of bites from any venomous creatures [16]. The use of medicinal plants against snakebites is a historical practice throughout the human history, and this knowledge has been transferred among the rural communities from generation after generation [17]. Nowadays, these herbal antidotes used in folk traditional medicine gained much attention by toxinologists worldwide as a tool for design of potent inhibitors against snake venom toxins. The potential advantages of antiophidic plants are their possible low cost, easy access, stability at room temperature, and ability to neutralize a broad spectrum of toxins, including the local tissue damage [12, 15-17].

So, the objective of this review is to provide an updated overview of medicinal plants used popularly as antiophidic and discuss the main species with pharmacological studies supporting the uses, with emphasis on plants inhibiting local effects of snake envenomation, since this is a critical effect of snake venoms that could lead to relevant sequel to victims. A review of the main botanical families popularly used as antiophidic is presented, including the main species and forms of popular use of them. Then, studies supporting their popular use are discussed, as well as the advantages of this kind of approach for treatment of snake venom accident.

\section{Methodology}

An extensive review of the literature was undertaken in different scientific sources, such as PubMed (https://www.ncbi .nlm.nih.gov/pubmed), Science Direct (http://www.sciencedirect.com/), Scopus (https://www.scopus.com/), Web of Science (http://www.webofknowledge.com/), "Literatura Latino-Americana e do Caribe em Ciências da Saúde" (LILACS) (http://lilacs.bvsalud.org/), Scientific Electronic Library Online (SciELO) (http://www.scielo.org/), Google Scholar (https://scholar.google.com.br/), Cochrane Library (http:// www.cochranelibrary.com/), and Centre for Reviews and Dissemination (CRD) (http://www.crd.york.ac.uk/CRDWeb).

The study database included original articles published in peer-reviewed journals, as well as books, thesis, dissertations, patents, and other reports covering antiophidic plants (ethnopharmacological surveys, original articles, or reviews), dated until December 2016. For the online search, where applicable, the following search strategy was employed: ("plant” OR "plants" OR "plant extract" OR "vegetal” OR "vegetal species" OR "vegetal extract” OR "traditional medicine" OR "alternative medicine" OR "complementary therapy" OR "natural medicine" OR "ethnopharmacology” OR "ethnobotany" OR "herbal medicine" OR "herb" OR "herbs" OR "decoction" OR “tea" OR "infusion" OR "macerate”) AND ("snake venom" OR "snake" OR "snakes" OR "snakebite” OR “snakebites” OR “antivenom” OR “antivenoms” OR “antivenom" OR “anti-venoms” OR "antivenin” OR “antivenins” OR “anti-venin” OR “anti-venins” OR “antiophidian” OR "antiophidic" OR “snake envenomation” OR "antitoxin” OR "antitoxins" OR "snake antidote” OR "snake antidotes" OR "snake venom neutralization" OR "snake venom inhibition" OR "snake toxins inhibition" OR "snake toxins neutralization" OR "viper” OR “viperidae" OR “crotalinae" OR “viperinae” OR "elapidae” OR "pit-viper" OR "bothrops” OR “jararaca" OR "crotalus" OR "micrurus" OR "lachesis" OR "cobra” OR “naja” OR "bitis” OR “vipera” OR “daboia” OR “trimeresus”).

All abstracts and/or full-text data were considered, without language restriction. Then, the publications covering ethnobotanical and/or pharmacological studies of antiophidic plants were selected and carefully analyzed. With the information gathered in these studies, the actual scenario of 
the use of plants against snake venom was pointed out. Main botanical families used, main countries where antiophidic plants are reported, and mode of use mostly employed in folk medicine were described. Regarding studies of pharmacological evidence, the snake species that were most studied, which plant species were tested and presented positive results, correlating with those species that also presented record of ethnopharmacological use, were also reported.

The accepted botanical name of each medicinal plant listed was confirmed in at least 2 botanical databases among the following ones: Flora do Brasil (http://www.floradobrasil .jbrj.gov.br/), Tropicos (http://www.tropicos.org/), The Plant List (http://www.theplantlist.org/), and NCBI Taxonomy Browser (https://www.ncbi.nlm.nih.gov/taxonomy). In some cases, where the same species was considered as different ones (different synonyms used) in different papers, the accepted name according to the botanical databases mentioned above was used in the present review, bringing the synonym used in the original work between parenthesis.

\section{Medicinal Plants as a Popular Source of Antidotes for Snakebites: Traditional Use}

According to the literature search performed, a lot of ethnopharmacological studies showing medicinal plants claimed as antiophidic were found. A summary of these vegetal species can be observed in Table 1 .

Along our survey were found 150 botanical families containing plants with reputation against snakebites, among which the most cited ones were the families Fabaceae, Asteraceae, Apocynaceae, Lamiaceae, Rubiaceae, Euphorbiaceae, Araceae, Malvaceae, and Acanthaceae (Figure 1(a)). In a cross-cultural comparison of medicinal floras used against snakebites, Molander et al. [80] identified five countries with a high number of antiophidic plants and representing different cultures, geography, and floristic zones: Brazil, Nicaragua, Nepal, China, and South Africa. From these countries, some "hot" families were identified, which were Apocynaceae, Lamiaceae, Rubiaceae, and Zingiberaceae [80], similar to the present review, except for the Zingiberaceae family which was not so reported in our survey.

Medicinal plants with reputation against snakebites are found all over the world, especially in tropical or subtropical regions of Asia, Americas, and Africa (Figure 2). This fact may be associated with richness of flora of these regions, as well as with relative need of complementary therapies to treat snakebites, considering geographical features that could limit the distribution and availability of the antivenoms in these areas.

As observed in Figure 3(a), leaves and roots are the parts of plants most used in folk medicine. Regarding the mode of use, the most frequent one is the topical application of the vegetal products directly on the place of the bite (Figure 3(b)). This is interesting especially in snake venoms that cause serious local tissue damage, such as Bothrops and Daboia species. Since these snakes produce intense local tissue damage, which has a very rapid onset, a topical treatment could be interesting for a rapid inhibitory action. On the other hand, interestingly, the use of some plant species is made by internal and external routes simultaneously, while for some other species the route of administration could be chosen among internal or external use. However, since in several cases this information is not clear, this differentiation was not considered in data tables. Regarding the mode of preparation, in general, paste and decoction were the most cited forms of use. However, for most of the plants enlisted, the information of mode of preparation was missing or confusing.

It is important to emphasize that these plant species, in addition to their use as antiophidic agents, present a series of another popular uses (data not shown) in popular medicine, mainly anti-inflammatory activity. For example, Jatropha gossypiifolia (Euphorbiaceae) has antiophidic, antiinflammatory, analgesic, antipyretic, healing, and antihemorrhagic uses, among others [81].

\section{Antivenom Activities of Extracts of Medicinal Plants against Snake Venom Induced Local Tissue Damage}

4.1. General Aspects. Until date, according to our database, only a few numbers (less than $20 \%$ ) of the species with reputation against snakebites were tested in preclinical assays with different snake venoms, which shows that there is still a great road for the study of antiophidic plants. From these tested plants which have popular use documented in our database, more than a half (almost 60\%) showed positive results, which shows that in fact ethnobotany could be a good tool for bioprospecting of plants with antiophidic activity. In addition, the fact that among the tested vegetal species very significative results were obtained strongly suggests the potentiality of these natural products as a future source for development of snake venom inhibitors.

The plant families with most vegetal species showing positive results in antiophidic tests were Fabaceae, Euphorbiaceae, Apocynaceae, Lamiaceae, Asteraceae, Malvaceae, Melastomaceae, and Sapindaceae (Figure 1(b)). Crossing the data of popular use (Figure 1(a)) and of positive activity (Figure 1(b)), we can highlight these families as "hot" ones, that is, families that might be preferred or prioritized in studies searching for antiophidic plants.

Snakes from the genus Naja, Bothrops, and Bitis were the most evaluated ones in these antiophidic assays. However, although Naja and Bitis comprise a large fraction of the studies, virtually most of them are only in vitro studies, dealing with the in vitro enzymatic inhibition of classes of venom toxins relevant to local tissue damage, such as phospholipases $\mathrm{A}_{2}\left(\mathrm{PLA}_{2} \mathrm{~s}\right)$, hyaluronidases (SVHs), and proteases. More particularly, the great majority of these studies with Naja and Bitis snakes are part of the work undertaken by Molander et al. [82], aiming to investigate whether plants used in traditional medicine systems would be active against necrosis-inducing enzymes of snake venoms, having tested a total of 226 extracts from 94 plants from the countries of Mali, Democratic Republic of Congo, and South Africa against $\mathrm{PLA}_{2}$, SVHs, and proteases from Bitis arietans and Naja nigricollis (see Tables 2 and 4). Studies evaluating the inhibitory action of medicinal plants against these enzymes 
TABLE 1: List of medicinal plants used against snakebites.

\begin{tabular}{|c|c|c|c|c|}
\hline Plant name & Countries & Parts used & Use & Reference(s) \\
\hline \multicolumn{5}{|l|}{ Acanthaceae } \\
\hline Acanthus arboreus & Sri Lanka & ND & I & {$[18]$} \\
\hline $\begin{array}{l}\text { Andrographis echioides (syn. } \\
\text { Indoneesiella echioides) }\end{array}$ & India & Shoot & ND & {$[19]$} \\
\hline Andrographis lineata & India & Flower, leaf & $\mathrm{I}$ & {$[20]$} \\
\hline Andrographis paniculata & India & Leaf, whole plant & $\mathrm{I}, \mathrm{E}$ & {$[16,20-26]$} \\
\hline Barleria cristata & India, Pakistan & Leaf, root, seed, whole plant & $\mathrm{E}$ & {$[17,19,25]$} \\
\hline Barleria lupulina & Sri Lanka & ND & I & [18] \\
\hline Blechum pyramidatum & Nicaragua & Leaf, whole plant & I & {$[27]$} \\
\hline Blepharis maderaspatensis & India & Leaf & I & {$[28]$} \\
\hline Clinacanthus nutans & India & Leaf & $\mathrm{E}$ & {$[20]$} \\
\hline $\begin{array}{l}\text { Dicliptera paniculata (syn. } \\
\text { Peristrophe paniculata) }\end{array}$ & India & Root, whole plant & $\mathrm{I}, \mathrm{E}$ & {$[24,25]$} \\
\hline Fittonia albivenis & Peru & Aerial parts & $\mathrm{E}$ & [29] \\
\hline Hygrophila auriculata & India, Sri Lanka & Seed & I & {$[18,23]$} \\
\hline $\begin{array}{l}\text { Justicia adhatoda (syn. Adhatoda } \\
\text { vasica) }\end{array}$ & $\begin{array}{l}\text { India, Pakistan, Sri } \\
\text { Lanka }\end{array}$ & Flower, leaf, root & $\mathrm{I}, \mathrm{E}$ & {$[16-18,30]$} \\
\hline Justicia calyculata & Kenya & Aerial parts & $\mathrm{E}$ & {$[31]$} \\
\hline Justicia gendarussa & Bangladesh & Leaf & I & {$[30,32]$} \\
\hline $\begin{array}{l}\text { Justicia japonica (syn. Justicia } \\
\text { simplex) }\end{array}$ & India & Leaf & I & {$[23]$} \\
\hline Justicia pectoralis* ${ }^{*}$ & Brazil & Leaf & I & [33] \\
\hline Justicia procumbens & Sri Lanka & ND & $\mathrm{E}$ & {$[18]$} \\
\hline Justicia secunda $a^{\#}$ & Colombia & Flower, leaf, root, whole plant & $\mathrm{I}, \mathrm{E}$ & {$[34,35]$} \\
\hline Rhinacanthus nasutus & India & Leaf, root & $\mathrm{I}, \mathrm{E}$ & {$[16,28]$} \\
\hline Thunbergia alata & Colombia & Flower, leaf & E & [34] \\
\hline Trichanthera gigantea & Colombia & Leaf, root & $\mathrm{E}$ & {$[34]$} \\
\hline \multicolumn{5}{|l|}{ Acoraceae } \\
\hline Acorus calamus & $\begin{array}{l}\text { Bangladesh, India, } \\
\text { Pakistan, Sri Lanka }\end{array}$ & Rhizome, root & $\mathrm{I}, \mathrm{E}$ & $\begin{array}{c}{[17,18,20,22,25,32,} \\
36]\end{array}$ \\
\hline \multicolumn{5}{|l|}{ Adoxaceae } \\
\hline Sambucus nigra & Spain & Flower & $\mathrm{E}$ & [37] \\
\hline \multicolumn{5}{|l|}{ Amaranthaceae } \\
\hline $\begin{array}{l}\text { Achyranthes aspera (syn. } \\
\text { Achyranthes porphyristachya) }\end{array}$ & $\begin{array}{l}\text { Bangladesh, Colombia, } \\
\text { India }\end{array}$ & $\begin{array}{l}\text { Fruit, inflorescence, leaf, root, } \\
\text { seed, stem, whole plant }\end{array}$ & $\mathrm{I}, \mathrm{E}$ & $\begin{array}{c}{[17,20,22,23,25,28,} \\
30,32,35,38-41]\end{array}$ \\
\hline Aerva lanata & India, Sri Lanka & Rhizome & I & {$[18,20]$} \\
\hline Aerva sanguinolenta & Bangladesh & Leaf & ND & {$[30]$} \\
\hline Alternanthera albotomentosa & Colombia & Leaf & $\mathrm{E}$ & {$[34]$} \\
\hline Alternanthera brasiliana & Brazil & Flower & I & {$[33]$} \\
\hline Alternanthera sessilis & Sri Lanka & $\mathrm{ND}$ & $\mathrm{I}$ & {$[18]$} \\
\hline Amaranthus blitum & India & Root & I & {$[25]$} \\
\hline Amaranthus dubius & Colombia & Fruit peel, leaf, root, seed & $\mathrm{E}$ & {$[34]$} \\
\hline Amaranthus polygonoides & Sri Lanka & ND & $\mathrm{E}$ & {$[18]$} \\
\hline Amaranthus spinosus & India & Leaf, root, stem, whole plant & $\mathrm{E}$ & {$[17,19,32,42]$} \\
\hline Amaranthus viridis & $\begin{array}{l}\text { Bangladesh, India, } \\
\text { Pakistan, Sri Lanka }\end{array}$ & Leaf, stem, whole plant & $\mathrm{E}$ & {$[17,18,30,42,43]$} \\
\hline Chenopodium album & $\begin{array}{l}\text { Bangladesh, India, } \\
\text { Pakistan }\end{array}$ & Fruit, root, whole plant & $\mathrm{E}$ & {$[17,32,41]$} \\
\hline Cyathula tomentosa & India & Leaf & $\mathrm{ND}$ & {$[19]$} \\
\hline $\begin{array}{l}\text { Dysphania ambrosioides (syn. } \\
\text { Chenopodium ambrosioides) }\end{array}$ & Colombia & Whole plant & $\mathrm{E}$ & {$[34]$} \\
\hline
\end{tabular}


TABle 1: Continued.

\begin{tabular}{|c|c|c|c|c|}
\hline Plant name & Countries & Parts used & Use & Reference(s) \\
\hline \multicolumn{5}{|l|}{ Amaryllidaceae } \\
\hline Allium ascalonicum & Sri Lanka & ND & I & {$[18]$} \\
\hline Allium cepa* & $\begin{array}{l}\text { Bangladesh, Colombia, } \\
\text { India, Kenya }\end{array}$ & Bulb, latex, leaf & $\mathrm{E}$ & {$[20,25,31,32,34,40]$} \\
\hline Allium sativum ${ }^{*}$ & $\begin{array}{l}\text { Colombia, India, Sri } \\
\text { Lanka, Spain }\end{array}$ & Bulb, inflorescence, leaf & $\mathrm{I}, \mathrm{E}$ & {$[18,22,23,37,44]$} \\
\hline Ammocharis tinneana & Kenya & Latex & ND & {$[31]$} \\
\hline Crinum asiaticum & Sri Lanka & ND & $\mathrm{E}$ & {$[18]$} \\
\hline Crinum latifolium & Sri Lanka & ND & $\mathrm{E}$ & [18] \\
\hline Hymenocallis littoralis & Nicaragua & Leaf, root & $\mathrm{I}, \mathrm{E}$ & {$[27]$} \\
\hline \multicolumn{5}{|l|}{ Anacardiaceae } \\
\hline Anacardium occidentale ${ }^{*}$ & India, Nicaragua & Bark, fruit, leaf, root & $\mathrm{I}, \mathrm{E}$ & {$[27,45]$} \\
\hline $\begin{array}{l}\text { Buchanania cochinchinensis (syn. } \\
\text { Buchanania lanzan) }\end{array}$ & India & Bark & $\mathrm{E}$ & {$[24,38]$} \\
\hline Mangifera indica* & $\begin{array}{c}\text { Bangladesh, Pakistan, Sri } \\
\text { Lanka }\end{array}$ & Leaf & $\mathrm{E}$ & {$[17,18,32]$} \\
\hline Mangifera minor & Papua New Guinea & Bark & I & {$[46]$} \\
\hline Pistacia chinensis & Pakistan & Gall & $\mathrm{E}$ & {$[17]$} \\
\hline $\begin{array}{l}\text { Pistacia chinensis subsp. } \\
\text { integerrima* }\end{array}$ & India, Pakistan & Gall, leaf & $\mathrm{E}$ & {$[17,19]$} \\
\hline Semecarpus anacardium & India & Root & I & {$[20]$} \\
\hline Semecarpus coriacea & Sri Lanka & ND & $\mathrm{E}$ & [18] \\
\hline Spondias dulcis & Sri Lanka & ND & $\mathrm{E}$ & [18] \\
\hline Spondias mombin ${ }^{*}$ & Peru & Bark & ND & [29] \\
\hline Tapirira guianensis & Colombia & Oil & $\mathrm{E}$ & {$[34]$} \\
\hline \multicolumn{5}{|l|}{ Annonaceae } \\
\hline Annona montana ${ }^{\#}$ & Brazil & Leaf & I & {$[33,47]$} \\
\hline Annona muricata & Brazil & Leaf & ND & {$[48]$} \\
\hline Annona senegalensis ${ }^{*}$ & Kenya & Leaf & $\mathrm{I}, \mathrm{E}$ & {$[31]$} \\
\hline Annona squamosa & Bangladesh, India & Bark, fruit & $\mathrm{I}, \mathrm{E}$ & {$[23,32]$} \\
\hline Polyalthia longifolia & Bangladesh & Whole plant & ND & {$[30]$} \\
\hline Uvaria scheffleri & Kenya & Leaf, root & $\mathrm{E}$ & {$[31]$} \\
\hline \multicolumn{5}{|l|}{ Apiaceae } \\
\hline Centella asiatica & Sri Lanka & ND & $\mathrm{E}$ & [18] \\
\hline Conium maculatum & Spain & Leaf & E & {$[37]$} \\
\hline Coriandrum sativum & Sri Lanka & $\mathrm{ND}$ & I & {$[18]$} \\
\hline Eryngium bourgatii & Spain & Aerial parts, root & $\mathrm{E}$ & {$[37]$} \\
\hline Eryngium campestre & Spain & Aerial parts, root & $\mathrm{E}$ & {$[37]$} \\
\hline Eryngium foetidum & Nicaragua, Sri Lanka & Leaf & $\mathrm{I}, \mathrm{E}$ & {$[18,27]$} \\
\hline Steganotaenia araliacea & Kenya & Root & $\mathrm{E}$ & {$[31]$} \\
\hline Trachyspermum ammi & Sri Lanka & ND & I & [18] \\
\hline Trachyspermum roxburghianum & Sri Lanka & ND & I & [18] \\
\hline \multicolumn{5}{|l|}{ Apocynaceae } \\
\hline Allamanda cathartica* & Colombia & Aerial parts, branch, leaf, stem & $\mathrm{I}, \mathrm{E}$ & {$[35,44]$} \\
\hline Alstonia scholaris & $\begin{array}{l}\text { Bangladesh, India, Sri } \\
\text { Lanka }\end{array}$ & Bark, flower, latex, leaf, root & $\mathrm{I}, \mathrm{E}$ & {$[18,19,32]$} \\
\hline Alstonia venenata & Sri Lanka & ND & $\mathrm{E}$ & {$[18]$} \\
\hline Asclepias curassavica & Nicaragua & $\begin{array}{l}\text { Bark, flower, latex, leaf, root, } \\
\text { whole plant }\end{array}$ & I, E & {$[27]$} \\
\hline Blepharodon mucronatum & Nicaragua & Leaf, whole plant & $\mathrm{I}, \mathrm{E}$ & {$[27]$} \\
\hline Calotropis acia & Sri Lanka & ND & $\mathrm{I}, \mathrm{E}$ & {$[18]$} \\
\hline
\end{tabular}


TABle 1: Continued.

\begin{tabular}{|c|c|c|c|c|}
\hline Plant name & Countries & Parts used & Use & Reference(s) \\
\hline Calotropis gigantea & $\begin{array}{l}\text { Bangladesh, India, Sri } \\
\text { Lanka }\end{array}$ & Latex, leaf, root & $\mathrm{I}, \mathrm{E}$ & $\begin{array}{c}{[16,18,20,22,23,28,} \\
32,38,49]\end{array}$ \\
\hline Calotropis procera* & $\begin{array}{l}\text { Bangladesh, India, } \\
\text { Pakistan }\end{array}$ & Flower, latex, leaf, root, shoot & $\mathrm{I}, \mathrm{E}$ & {$[17,25,32,38,40,41]$} \\
\hline $\begin{array}{l}\text { Cascabela thevetia (syn. Thevetia } \\
\text { peruviana) }\end{array}$ & Brazil & Bark, seed & $\mathrm{E}$ & {$[50]$} \\
\hline Catharanthus roseus & Bangladesh, Colombia & Flower, leaf & $\mathrm{I}, \mathrm{E}$ & {$[32,34]$} \\
\hline Cerbera floribunda & Papua New Guinea & Latex & $\mathrm{E}$ & [46] \\
\hline Cerbera odollam & Sri Lanka & ND & $\mathrm{E}$ & {$[18]$} \\
\hline $\begin{array}{l}\text { Cryptolepis dubia (syn. Cryptolepis } \\
\text { buchanani) }\end{array}$ & India, Sri Lanka & Root & ND & {$[18,19]$} \\
\hline $\begin{array}{l}\text { Cynanchum viminale (syn. } \\
\text { Sarcostemma viminale) }\end{array}$ & India & Whole plant & $\mathrm{E}$ & {$[38]$} \\
\hline $\begin{array}{l}\text { Dregea volubilis (syn. Wattakaka } \\
\text { volubilis) }\end{array}$ & India, Sri Lanka & Root & $\mathrm{I}, \mathrm{E}$ & {$[18,23]$} \\
\hline Echidnopsis dammanniana & Ethiopia & Stem & $\mathrm{E}$ & {$[51]$} \\
\hline Echites umbellatus & Nicaragua & Root & I & {$[27]$} \\
\hline Gymnema sylvestre & India & Leaf, root & $\mathrm{I}, \mathrm{E}$ & {$[19,20,22,23,28,52]$} \\
\hline Hemidesmus indicus ${ }^{*}$ & $\begin{array}{l}\text { Bangladesh, India, Sri } \\
\text { Lanka }\end{array}$ & Root, whole plant & $\mathrm{I}, \mathrm{E}$ & $\begin{array}{c}{[18,20,22,25,28,32,} \\
38]\end{array}$ \\
\hline $\begin{array}{l}\text { Holarrhena pubescens (syn. } \\
\text { Holarrhena antidysenterica) }\end{array}$ & Bangladesh, India & Bark, root, stem & $\mathrm{I}, \mathrm{E}$ & {$[24,32,38]$} \\
\hline Hoya ovalifolia & Sri Lanka & ND & I & {$[18]$} \\
\hline Hunteria zeylanica & Sri Lanka & $\mathrm{ND}$ & $\mathrm{E}$ & {$[18]$} \\
\hline Ichnocarpus frutescens & Bangladesh & Root & I & {$[32]$} \\
\hline $\begin{array}{l}\text { Nerium oleander (syn. Nerium } \\
\text { indicum) }\end{array}$ & $\begin{array}{l}\text { India, Pakistan, Sri } \\
\text { Lanka }\end{array}$ & Leaf, root, seed & $\mathrm{E}$ & {$[17,18,20,28,41,53]$} \\
\hline Odontadenia puncticulosa & Nicaragua & Leaf & I & [27] \\
\hline Pergularia daemia & India, Namibia & Leaf & I & {$[19,28,54]$} \\
\hline Rauvolfia serpentina & $\begin{array}{l}\text { Bangladesh, India, Sri } \\
\text { Lanka }\end{array}$ & Flower, leaf, rhizome, root, seed & $\mathrm{I}, \mathrm{E}$ & {$[18,20,22,28,30,32]$} \\
\hline $\begin{array}{l}\text { Rauvolfia tetraphylla (syn. Rauvolfia } \\
\text { canescens) }\end{array}$ & Bangladesh, India & Root & $\mathrm{E}$ & {$[16,30]$} \\
\hline Tabernaemontana dichotoma & Sri Lanka & ND & $\mathrm{E}$ & {$[18]$} \\
\hline Tabernaemontana divaricata & Sri Lanka & ND & I & {$[18]$} \\
\hline Tabernaemontana sananho & Peru & Leaf & $\mathrm{E}$ & [29] \\
\hline Tylophora indica* ${ }^{*}$ & Bangladesh, India & Leaf & $\mathrm{I}$ & {$[23,30,32]$} \\
\hline Tylophora longifolia & India & Flower, leaf & ND & {$[20]$} \\
\hline Vincetoxicum hirundinaria & India & Root & ND & [19] \\
\hline Willughbeia edulis & Bangladesh & Stem & $\mathrm{E}$ & {$[32]$} \\
\hline Wrightia antidysenterica & Sri Lanka & ND & $\mathrm{E}$ & {$[18]$} \\
\hline Wrightia arborea & India & Bark & ND & {$[19]$} \\
\hline Wrightia tinctoria & India & Leaf & ND & {$[38]$} \\
\hline \multicolumn{5}{|l|}{ Aponogetonaceae } \\
\hline Aponogeton crispus & Sri Lanka & ND & $\mathrm{E}$ & {$[18]$} \\
\hline \multicolumn{5}{|l|}{ Araceae } \\
\hline Alocasia cucullata ${ }^{\#}$ & Colombia & Rhizome, root & $\mathrm{E}$ & {$[35,44]$} \\
\hline Amorphophallus commutatus & India & Tuber & ND & {$[55]$} \\
\hline Amorphophallus paeoniifolius & Sri Lanka & ND & I & {$[18]$} \\
\hline Anaphyllum beddomei & India & Rhizome & $\mathrm{E}$ & {$[16]$} \\
\hline Anthurium marmoratum & Colombia & Branch, leaf, stem & $\mathrm{E}$ & {$[35]$} \\
\hline Arisaema concinnum & India & Fruit, tuber & $\mathrm{ND}$ & [19] \\
\hline
\end{tabular}


TABle 1: Continued.

\begin{tabular}{|c|c|c|c|c|}
\hline Plant name & Countries & Parts used & Use & Reference(s) \\
\hline Arisaema flavum & Pakistan & Rhizome & ND & [17] \\
\hline Arisaema jacquemontii & India, Pakistan & Flower, leaf, tuber & ND & {$[17,19,56]$} \\
\hline Arisaema tortuosum & India & Bulb, tuber & I & {$[38,55]$} \\
\hline Caladium bicolor & Peru & Tuber & $\mathrm{E}$ & [57] \\
\hline Dieffenbachia longispatha ${ }^{\#}$ & Colombia & Whole plant & I, E & [35] \\
\hline Dieffenbachia parlatorei & Colombia & Root & $\mathrm{E}$ & {$[44]$} \\
\hline Dracontium croatii & Colombia & Rhizome & $\mathrm{I}, \mathrm{E}$ & {$[35]$} \\
\hline Dracontium spruceanum & Colombia, Peru & Stem, tuber, root & $\mathrm{E}$ & {$[29,34,44,57]$} \\
\hline Dracunculus vulgaris & Spain & Bulb, flower & $\mathrm{E}$ & {$[37]$} \\
\hline Homalomena aromatica & Bangladesh & Rhizome & $\mathrm{E}$ & {$[32]$} \\
\hline Homalomena peltata & Colombia & Leaf & $\mathrm{E}$ & {$[44]$} \\
\hline Homalomena picturata & Colombia & Leaf & $\mathrm{E}$ & {$[34]$} \\
\hline Lasia spinosa & Sri Lanka & ND & $\mathrm{E}$ & {$[18]$} \\
\hline Philodendron deltoideum & Peru & Aerial parts & I, E & [29] \\
\hline Philodendron hederaceum & Nicaragua & Leaf, stem & I, E & {$[27]$} \\
\hline Philodendron heleniae & Colombia & Leaf & E & {$[44]$} \\
\hline Philodendron megalophyllum* & Brazil & Vine & I & {$[33]$} \\
\hline Philodendron tripartitum ${ }^{*}$ & Colombia & Branch, leaf & $\mathrm{E}$ & {$[35]$} \\
\hline Pothos scandens & Sri Lanka & ND & I & {$[18]$} \\
\hline Rhodospatha oblongata & Colombia & Rhizome & $\mathrm{E}$ & {$[35]$} \\
\hline Sauromatum venosum & India, Pakistan & Leaf, tuber & I, E & {$[17,38]$} \\
\hline Typhonium roxburghii & Sri Lanka & ND & I & [18] \\
\hline Xanthosoma poeppigii & Peru & Stem & $\mathrm{E}$ & [57] \\
\hline \multicolumn{5}{|l|}{ Araliaceae } \\
\hline Osmoxylon micranthum & Papua New Guinea & Latex & $\mathrm{E}$ & {$[46]$} \\
\hline \multicolumn{5}{|l|}{ Arecaceae } \\
\hline Areca catechu & Sri Lanka & ND & $\mathrm{E}$ & {$[18]$} \\
\hline Caryota urens & Sri Lanka & ND & I & {$[18]$} \\
\hline Cocos nucifera & Sri Lanka & ND & $\mathrm{I}$ & {$[18]$} \\
\hline Corypha umbraculifera & Sri Lanka & ND & $\mathrm{E}$ & {$[18]$} \\
\hline Euterpe edulis & Brazil & Latex & $\mathrm{E}$ & {$[50]$} \\
\hline Euterpe oleracea & Brazil & Fruit & $\mathrm{E}$ & [33] \\
\hline Phoenix pusilla & Sri Lanka & ND & I & {$[18]$} \\
\hline Syagrus coronata & Brazil & Bark & ND & [47] \\
\hline \multicolumn{5}{|l|}{ Aristolochiaceae } \\
\hline Aristolochia birostris & Brazil & Whole plant & $\mathrm{I}$ & {$[47]$} \\
\hline Aristolochia bracteolata ${ }^{*}$ & India, Sri Lanka & Fruit, leaf, whole plant & I, E & {$[18,23,38,55]$} \\
\hline Aristolochia clematitis & Serbia & Rhizome & ND & {$[58]$} \\
\hline Aristolochia cordiflora & Colombia & Leaf, stem & $\mathrm{I}, \mathrm{E}$ & {$[34,44]$} \\
\hline Aristolochia grandiflora & Colombia & Whole plant & $\mathrm{I}, \mathrm{E}$ & {$[35]$} \\
\hline Aristolochia indica* & Bangladesh, India & Leaf, root, whole plant & $\mathrm{I}, \mathrm{E}$ & $\begin{array}{c}{[16,20,22,23,28,30,} \\
32]\end{array}$ \\
\hline Aristolochia ovalifolia & Mexico & Root & ND & {$[59]$} \\
\hline Aristolochia pilosa & Colombia & Root & $\mathrm{I}, \mathrm{E}$ & {$[35]$} \\
\hline Aristolochia tagala & India & Whole plant & $\mathrm{I}, \mathrm{E}$ & {$[16]$} \\
\hline Aristolochia trilobata & Brazil, Nicaragua & Leaf, root, whole plant & I, E & {$[27,50]$} \\
\hline Thottea siliquosa & India & Leaf, root & $\mathrm{E}$ & {$[16,26]$} \\
\hline \multicolumn{5}{|l|}{ Asparagaceae } \\
\hline Asparagus racemosus & Bangladesh, Sri Lanka & Leaf, root & $\mathrm{E}$ & {$[18,30,32]$} \\
\hline Drimia indica (syn. Urginea indica) & India & Bulb & $\mathrm{E}$ & {$[25]$} \\
\hline
\end{tabular}


TABle 1: Continued.

\begin{tabular}{|c|c|c|c|c|}
\hline Plant name & Countries & Parts used & Use & Reference(s) \\
\hline Peliosanthes teta & Bangladesh & Root & $\mathrm{E}$ & {$[32]$} \\
\hline Sansevieria parva & Kenya & Latex & $\mathrm{E}$ & {$[31]$} \\
\hline Sansevieria roxburghiana & India & Rhizome & $\mathrm{I}$ & [23] \\
\hline Sansevieria trifasciata & Bangladesh, Colombia & Aerial parts, whole plant & $\mathrm{E}$ & {$[30,34,60]$} \\
\hline Sansevieria zeylanica & Sri Lanka & ND & $\mathrm{E}$ & [18] \\
\hline \multicolumn{5}{|l|}{ Aspleniaceae } \\
\hline $\begin{array}{l}\text { Asplenium dalhousiae (syn. Ceterach } \\
\text { dalhousiae) }\end{array}$ & Pakistan & Leaf & ND & {$[17]$} \\
\hline \multicolumn{5}{|l|}{ Asteraceae } \\
\hline Achillea millefolium & India & Whole plant & I & {$[20]$} \\
\hline $\begin{array}{l}\text { Acmella paniculata (syn. Spilanthes } \\
\text { paniculata) }\end{array}$ & Sri Lanka & ND & I & {$[18]$} \\
\hline Adenostemma fosbergii & Ecuador & Leaf & I & {$[61]$} \\
\hline Adenostemma lavenia ${ }^{\#}$ & Colombia & Whole plant & $\mathrm{E}$ & {$[35]$} \\
\hline Ageratum conyzoides ${ }^{\#}$ & $\begin{array}{l}\text { Colombia, India, } \\
\text { Bangladesh }\end{array}$ & Flower, leaf, root & $\mathrm{E}$ & {$[19,24,32,34]$} \\
\hline Ageratum houstonianum & Pakistan & Inflorescence, leaf & $\mathrm{E}$ & {$[17]$} \\
\hline $\begin{array}{l}\text { Ambrosia peruviana (syn. Ambrosia } \\
\text { cumanensis) }\end{array}$ & Colombia & Aerial parts, whole plant & I, E & {$[34,44]$} \\
\hline Artemisia maritima & Pakistan & Whole plant & $\mathrm{E}$ & {$[17]$} \\
\hline Artemisia scoparia & India, Pakistan & Whole plant & $\mathrm{E}$ & {$[17,40]$} \\
\hline Austroeupatorium inulifolium & Colombia & Leaf & $\mathrm{E}$ & {$[34,44]$} \\
\hline $\begin{array}{l}\text { Ayapana triplinervis (syn. } \\
\text { Eupatorium ayapana, Eupatorium } \\
\text { triplinerve) }\end{array}$ & Brazil & Leaf & I & {$[33,50]$} \\
\hline $\begin{array}{l}\text { Baccharis inamoena (syn. Baccharis } \\
\text { trinervis) }\end{array}$ & Colombia & Aerial parts, whole plant & $\mathrm{E}$ & {$[34]$} \\
\hline $\begin{array}{l}\text { Baccharoides anthelmintica (syn. } \\
\text { Centratherum anthelminticum) }\end{array}$ & India & Seed & ND & {$[26]$} \\
\hline Bidens biternata & India & Leaf & $\mathrm{E}$ & [25] \\
\hline Bidens pilosa & Kenya & Leaf & $\mathrm{E}$ & {$[31]$} \\
\hline Blumea axillaris & Sri Lanka & $\mathrm{ND}$ & I & {$[18]$} \\
\hline $\begin{array}{l}\text { Blumea brevipes (syn. Laggera } \\
\text { brevipes) }\end{array}$ & Kenya & Root & ND & {$[31]$} \\
\hline Calendula officinalis & India & Flower & I & {$[20,28]$} \\
\hline Chromolaena odorata & Colombia & Whole plant & $\mathrm{E}$ & [34] \\
\hline Clibadium sylvestre ${ }^{\#}$ & Colombia & Whole plant & $\mathrm{I}, \mathrm{E}$ & {$[35]$} \\
\hline Conyza sumatrensis & Kenya & Leaf & I & {$[31]$} \\
\hline Cyanthillium cinereum & Sri Lanka & ND & $\mathrm{E}$ & {$[18]$} \\
\hline Eclipta prostrata (syn. Eclipta alba)* & $\begin{array}{l}\text { Bangladesh, India, } \\
\text { Pakistan, Sri Lanka }\end{array}$ & Leaf, whole plant & I, E & {$[17,18,20,28,30,40]$} \\
\hline Elephantopus scaber & Sri Lanka & ND & $\mathrm{E}$ & {$[18]$} \\
\hline Emilia sonchifolia & $\begin{array}{l}\text { Bangladesh, Colombia, } \\
\text { India, Sri Lanka }\end{array}$ & Leaf, whole plant & $\mathrm{I}, \mathrm{E}$ & {$[16,18,30,34]$} \\
\hline Erechtites valerianifolia ${ }^{\#}$ & Colombia & Branch, leaf, stem & $\mathrm{I}, \mathrm{E}$ & {$[35]$} \\
\hline Gnaphalium purpureum & Sri Lanka & ND & I, E & {$[18]$} \\
\hline Gynura hispida & Sri Lanka & ND & I & {$[18]$} \\
\hline Helianthus annuus & India & Seed & $\mathrm{E}$ & {$[20]$} \\
\hline Inula helenium & Serbia & Root & $\mathrm{E}$ & {$[58]$} \\
\hline Laggera alata & Sri Lanka & ND & $\mathrm{E}$ & {$[18]$} \\
\hline Linzia glabra (syn. Vernonia glabra) & Kenya & Leaf & $\mathrm{E}$ & {$[31]$} \\
\hline Microglossa pyrifolia & Kenya & Leaf & $\mathrm{E}$ & {$[31]$} \\
\hline
\end{tabular}


TABle 1: Continued.

\begin{tabular}{|c|c|c|c|c|}
\hline Plant name & Countries & Parts used & Use & Reference(s) \\
\hline Mikania cordata & Bangladesh & Leaf & $\mathrm{E}$ & {$[32]$} \\
\hline Mikania cordifolia & Nicaragua & Leaf, stem, whole plant & $\mathrm{I}, \mathrm{E}$ & [27] \\
\hline Mikania guaco & Colombia, Nicaragua & Leaf, stem, whole plant & I, E & {$[27,35,44]$} \\
\hline Neurolaena lobata* & Colombia, Nicaragua & Aerial parts, branch, leaf, stem & $\mathrm{I}, \mathrm{E}$ & {$[27,35,44]$} \\
\hline Pentanema indicum & India, Sri Lanka & Leaf, root & I & {$[18,23]$} \\
\hline Pluchea indica* ${ }^{*}$ & India & Flower, seed & I, E & {$[20]$} \\
\hline Pseudelephantopus spicatus* & Colombia & Whole plant & $\mathrm{E}$ & {$[44]$} \\
\hline Saussurea simpsoniana & India & Flower & ND & {$[19]$} \\
\hline Senecio chrysanthemoides & Pakistan & Whole plant & $\mathrm{E}$ & {$[17]$} \\
\hline $\begin{array}{l}\text { Seriphidium brevifolium (syn. } \\
\text { Artemisia brevifolia) }\end{array}$ & Pakistan & Flower, leaf & $\mathrm{E}$ & {$[17]$} \\
\hline Solanecio mannii & Kenya & Leaf & $\mathrm{E}$ & {$[31]$} \\
\hline Sphaeranthus africanus & Sri Lanka & ND & I & {$[18]$} \\
\hline Sphaeranthus indicus & Sri Lanka & $\mathrm{ND}$ & $\mathrm{I}$ & {$[18]$} \\
\hline Sphagneticola trilobata & Nicaragua & Flower, leaf, stem, whole plant & I & {$[27]$} \\
\hline Tagetes minuta & Kenya & Leaf & $\mathrm{E}$ & [31] \\
\hline Taraxacum officinale & Colombia, Pakistan & Leaf, root, whole plant & I, E & {$[17,34]$} \\
\hline Tithonia diversifolia & Colombia, Kenya & Leaf, whole plant & $\mathrm{I}, \mathrm{E}$ & {$[31,34]$} \\
\hline Tricholepis glaberrima & India & Root & ND & [19] \\
\hline Verbesina gigantea & Colombia & Root, stem & $\mathrm{I}, \mathrm{E}$ & {$[34]$} \\
\hline Vernonanthura patens & Colombia & Whole plant & $\mathrm{E}$ & {$[34]$} \\
\hline Vernonia zeylanicum & Sri Lanka & ND & $\mathrm{I}, \mathrm{E}$ & {$[18]$} \\
\hline Wedelia calendulacea & India & Leaf & I & {$[20]$} \\
\hline $\begin{array}{l}\text { Wollastonia biflora (syn. Wedelia } \\
\text { biflora) }\end{array}$ & Sri Lanka & ND & $\mathrm{E}$ & {$[18]$} \\
\hline Xanthium strumarium & Pakistan & Aerial parts & $\mathrm{E}$ & {$[17]$} \\
\hline \multicolumn{5}{|l|}{ Balsaminaceae } \\
\hline Impatiens balsamina & Colombia & Flower & I, E & {$[34]$} \\
\hline \multicolumn{5}{|l|}{ Begoniaceae } \\
\hline $\begin{array}{l}\text { Begonia annulata (syn. Begonia } \\
\text { barbata) }\end{array}$ & Bangladesh & Leaf, stem & $\mathrm{E}$ & {$[32]$} \\
\hline \multicolumn{5}{|l|}{ Berberidaceae } \\
\hline Dysosma pleiantha & China, Taiwan & Rhizome & ND & {$[62]$} \\
\hline \multicolumn{5}{|l|}{ Betulaceae } \\
\hline Betula alnoides & India & Bark, leaf & ND & {$[19]$} \\
\hline \multicolumn{5}{|l|}{ Bignoniaceae } \\
\hline Crescentia cujete $^{\#}$ & Colombia & Fruit & I & [35] \\
\hline $\begin{array}{l}\text { Dolichandra unguis-cati (syn. } \\
\text { Macfadyena unguis-cati) }\end{array}$ & Colombia & Whole plant & $\mathrm{E}$ & {$[35]$} \\
\hline $\begin{array}{l}\text { Handroanthus barbatus (syn. } \\
\text { Tabebuia barbata) }\end{array}$ & Brazil & Leaf & I & {$[33]$} \\
\hline Mansoa alliacea & Peru & Bark, root & I & [57] \\
\hline Oroxylum indicum & Bangladesh, Sri Lanka & Bark & $\mathrm{E}$ & {$[18,32]$} \\
\hline Stereospermum chelonoides & Sri Lanka & ND & I & [18] \\
\hline Stereospermum colais & Sri Lanka & ND & $\mathrm{E}$ & {$[18]$} \\
\hline Tabebuia rosea* & Colombia & Bark & $\mathrm{I}, \mathrm{E}$ & {$[35]$} \\
\hline \multicolumn{5}{|l|}{ Bixaceae } \\
\hline Bixa orellana* & $\begin{array}{l}\text { Bangladesh, Colombia, } \\
\text { Nicaragua }\end{array}$ & $\begin{array}{l}\text { Branch, fruit, latex, leaf, root, } \\
\text { stem }\end{array}$ & $\mathrm{I}, \mathrm{E}$ & {$[27,32]$} \\
\hline Cochlospermum vitifolium & Colombia & Aerial parts & $\mathrm{E}$ & {$[34]$} \\
\hline
\end{tabular}


TABle 1: Continued.

\begin{tabular}{|c|c|c|c|c|}
\hline Plant name & Countries & Parts used & Use & Reference(s) \\
\hline \multicolumn{5}{|l|}{ Boraginaceae } \\
\hline $\begin{array}{l}\text { Cordia dichotoma (syn. Cordia } \\
\text { obliqua) }\end{array}$ & Pakistan & Bark, fruit & ND & {$[17]$} \\
\hline $\begin{array}{l}\text { Cordia spinescens (syn. Varronia } \\
\text { spinescens) }\end{array}$ & Colombia & Leaf & $\mathrm{E}$ & {$[34]$} \\
\hline Cynoglossum zeylanicum & India & Root & I & {$[63]$} \\
\hline Echium vulgare & Spain & Aerial parts & ND & [37] \\
\hline $\begin{array}{l}\text { Ehretia microphylla (syn. Ehretia } \\
\text { buxifolia) }\end{array}$ & India, Sri Lanka & Root & $\mathrm{I}, \mathrm{E}$ & {$[18,20]$} \\
\hline Heliotropium europaeum & Pakistan & Whole plant & $\mathrm{E}$ & {$[17]$} \\
\hline Heliotropium indicum ${ }^{\#}$ & Nicaragua & Leaf, whole plant & I & {$[27]$} \\
\hline Tournefortia cuspidata ${ }^{\#}$ & Colombia & Branch, leaf, stem & $\mathrm{E}$ & {$[35]$} \\
\hline Trichodesma indicum ${ }^{*}$ & Pakistan & Leaf, root & ND & {$[17]$} \\
\hline Trichodesma zeylanicum & India & Root & $\mathrm{I}, \mathrm{E}$ & {$[20]$} \\
\hline \multicolumn{5}{|l|}{ Brassicaceae } \\
\hline Brassica juncea & Sri Lanka & ND & $\mathrm{E}$ & {$[18]$} \\
\hline $\begin{array}{l}\text { Brassica rapa (syn. Brassica } \\
\text { campestris) }\end{array}$ & India & ND & $\mathrm{E}$ & {$[25]$} \\
\hline Lepidium virginicum & Colombia & Whole plant & $\mathrm{E}$ & {$[34]$} \\
\hline \multicolumn{5}{|l|}{ Bromeliaceae } \\
\hline Ananas comosus & Nicaragua, Sri Lanka & Flower, leaf, root & $\mathrm{I}, \mathrm{E}$ & {$[18,27]$} \\
\hline Bromelia pinguin & Nicaragua & Leaf & $\mathrm{I}, \mathrm{E}$ & [27] \\
\hline \multicolumn{5}{|l|}{ Burseraceae } \\
\hline Boswellia serrata & India & Bark & I & {$[24]$} \\
\hline Bursera simaruba & Nicaragua & Bark, whole plant & I & [27] \\
\hline Canarium zeylanicum & Sri Lanka & ND & $\mathrm{E}$ & {$[18]$} \\
\hline \multicolumn{5}{|l|}{ Cactaceae } \\
\hline $\begin{array}{l}\text { Opuntia ficus-indica (syn. Opuntia } \\
\text { vulgaris) }\end{array}$ & India & Root & ND & {$[25]$} \\
\hline Pereskia bleo ${ }^{\#}$ & Colombia & Leaf, stem & $\mathrm{E}$ & {$[35]$} \\
\hline \multicolumn{5}{|l|}{ Calophyllaceae } \\
\hline Calophyllum inophyllum & Sri Lanka & ND & $\mathrm{E}$ & {$[18]$} \\
\hline Mesua ferrea & Sri Lanka & $\mathrm{ND}$ & $\mathrm{I}, \mathrm{E}$ & {$[18]$} \\
\hline \multicolumn{5}{|l|}{ Campanulaceae } \\
\hline Hippobroma longiflora & Nicaragua & Leaf, root, whole plant & $\mathrm{I}, \mathrm{E}$ & {$[27]$} \\
\hline \multicolumn{5}{|l|}{ Cannabaceae } \\
\hline Cannabis sativa & India, Sri Lanka & $\mathrm{ND}$ & I & {$[18,40]$} \\
\hline \multicolumn{5}{|l|}{ Cannaceae } \\
\hline Canna indica & Sri Lanka & ND & $\mathrm{E}$ & {$[18]$} \\
\hline \multicolumn{5}{|l|}{ Capparaceae } \\
\hline Capparis decidua & Pakistan & Flower, shoot & $\mathrm{E}$ & {$[17]$} \\
\hline Capparis moonii & Sri Lanka & ND & I & {$[18]$} \\
\hline Capparis roxburghii & Sri Lanka & ND & $\mathrm{E}$ & {$[18]$} \\
\hline Capparis zeylanica & Sri Lanka & ND & $\mathrm{I}, \mathrm{E}$ & {$[18]$} \\
\hline Carica papaya & India & Fruit & ND & {$[41]$} \\
\hline Crateva adansonii & Sri Lanka & ND & I & {$[18]$} \\
\hline $\begin{array}{l}\text { Crateva tapia (syn. Crateva } \\
\text { benthamii) }\end{array}$ & Brazil & Leaf & E & {$[33]$} \\
\hline $\begin{array}{l}\text { Cynophalla flexuosa (syn. Capparis } \\
\text { flexuosa) }\end{array}$ & Brazil & Bark & I & {$[64]$} \\
\hline
\end{tabular}


TABLE 1: Continued.

\begin{tabular}{|c|c|c|c|c|}
\hline Plant name & Countries & Parts used & Use & Reference(s) \\
\hline \multicolumn{5}{|l|}{ Caprifoliaceae } \\
\hline Nardostachys jatamansi & India & Root & ND & [19] \\
\hline Valeriana jatamansi & Pakistan, Sri Lanka & Root & I, E & {$[17,18]$} \\
\hline \multicolumn{5}{|l|}{ Celastraceae } \\
\hline Cassine glauca & India, Sri Lanka & Leaf & I & {$[18,19]$} \\
\hline Celastrus paniculatus & India & Bark, root, seed & I & {$[19,38]$} \\
\hline Gymnosporia emarginata & Sri Lanka & ND & I & [18] \\
\hline Parnassia nubicola & India & Tuber & ND & [19] \\
\hline \multicolumn{5}{|l|}{ Chrysobalanaceae } \\
\hline Parinari capensis & Namibia & Root & ND & [65] \\
\hline \multicolumn{5}{|l|}{ Cleomaceae } \\
\hline Cleome gynandra & Sri Lanka & ND & E & {$[18]$} \\
\hline Cleome viscosa & Sri Lanka & ND & I & {$[18]$} \\
\hline \multicolumn{5}{|l|}{ Clusiaceae } \\
\hline Garcinia morella & Sri Lanka & ND & I, E & {$[18]$} \\
\hline Garcinia xanthochymus & Sri Lanka & ND & I, E & {$[18]$} \\
\hline \multicolumn{5}{|l|}{ Colchicaceae } \\
\hline Gloriosa superba* & $\begin{array}{l}\text { India, Pakistan, Sri } \\
\text { Lanka }\end{array}$ & Tuber & I, E & {$[17,18,20,28,38,40]$} \\
\hline \multicolumn{5}{|l|}{ Combretaceae } \\
\hline Anogeissus latifolia & Bangladesh, India & Bark, whole plant & I, E & {$[25,30,38]$} \\
\hline Combretum collinum & Kenya & Root & E & [31] \\
\hline Combretum molle* & Kenya & Bark, root & I & {$[31]$} \\
\hline $\begin{array}{l}\text { Getonia floribunda (syn. } \\
\text { Calycopteris floribunda) }\end{array}$ & Bangladesh & Root & E & {$[32]$} \\
\hline Terminalia arjuna* & Bangladesh, India & Bark & I, E & {$[20,32]$} \\
\hline Terminalia bellirica & Sri Lanka & ND & I & [18] \\
\hline Terminalia chebula & Sri Lanka & ND & I & {$[18]$} \\
\hline \multicolumn{5}{|l|}{ Commelinaceae } \\
\hline Callisia gracilis & Colombia & Flower, leaf & I, E & [34] \\
\hline Commelina benghalensis & India, Sri Lanka & Root & ND & {$[18,42]$} \\
\hline \multicolumn{5}{|l|}{ Connaraceae } \\
\hline Connarus favosus ${ }^{*}$ & Brazil & Bark & I & [33] \\
\hline Connarus monocarpus & Sri Lanka & $\mathrm{ND}$ & E & {$[18]$} \\
\hline \multicolumn{5}{|l|}{ Convolvulaceae } \\
\hline $\begin{array}{l}\text { Argyreia nervosa (syn. Argyreia } \\
\text { speciosa) }\end{array}$ & India & Root, seed & ND & [19] \\
\hline Argyreia populifolia & Sri Lanka & ND & I & {$[18]$} \\
\hline Cuscuta reflexa & Sri Lanka & ND & $\mathrm{E}$ & {$[18]$} \\
\hline Dichondra repens & Kenya & Leaf & $\mathrm{E}$ & [31] \\
\hline Evolvulus alsinoides & India, Sri Lanka & Root & I & {$[18,23]$} \\
\hline Ipomoea alba & Sri Lanka & ND & E & {$[18]$} \\
\hline Ipomoea aquatica & Bangladesh & Leaf, whole plant & ND & [30] \\
\hline Ipomoea asarifolia & Sri Lanka & ND & I, E & {$[18]$} \\
\hline Ipomoea cairica $^{\#}$ & Colombia & Branch, leaf, stem & $\mathrm{E}$ & [35] \\
\hline Ipomoea mauritiana & Nicaragua & Leaf & $\mathrm{I}, \mathrm{E}$ & [27] \\
\hline Ipomoea pes-caprae & Nicaragua & Leaf, seed & I & [27] \\
\hline Ipomoea pes-tigridis & India, Sri Lanka & Root & I, E & {$[18,19,24,39]$} \\
\hline Ipomoea setifera & Nicaragua & Leaf & I, E & [27] \\
\hline
\end{tabular}


TABLe 1: Continued.

\begin{tabular}{|c|c|c|c|c|}
\hline Plant name & Countries & Parts used & Use & Reference(s) \\
\hline Ipomoea triloba & Sri Lanka & ND & I & {$[18]$} \\
\hline Operculina pteripes & Nicaragua & Leaf & $\mathrm{E}$ & {$[27]$} \\
\hline Rivea hypocrateriformis & India & $\mathrm{ND}$ & $\mathrm{I}$ & {$[24]$} \\
\hline \multicolumn{5}{|l|}{ Cornaceae } \\
\hline Alangium salviifolium & India & Bark & I & {$[20,23]$} \\
\hline \multicolumn{5}{|l|}{ Costaceae } \\
\hline $\begin{array}{l}\text { Cheilocostus speciosus (syn. Costus } \\
\text { speciosus) }\end{array}$ & $\begin{array}{l}\text { Bangladesh, India, Sri } \\
\text { Lanka }\end{array}$ & Bulb, leaf, stem, root, tuber & I, E & {$[18,19,32,55]$} \\
\hline Costus guanaiensis ${ }^{\#}$ & Colombia & Stem & $\mathrm{I}, \mathrm{E}$ & {$[35]$} \\
\hline Costus lasius ${ }^{*}$ & Colombia & Branch, leaf, stem & $\mathrm{I}, \mathrm{E}$ & {$[35]$} \\
\hline Costus lima & Colombia & Stem & $\mathrm{E}$ & {$[34]$} \\
\hline \multicolumn{5}{|l|}{ Crassulaceae } \\
\hline $\begin{array}{l}\text { Bryophyllum pinnatum (syn. } \\
\text { Kalanchoe pinnata) }\end{array}$ & India & Leaf & ND & {$[22,42]$} \\
\hline $\begin{array}{l}\text { Kalanchoe laciniata (syn. Kalanchoe } \\
\text { brasiliensis) }\end{array}$ & Brazil & Leaf & $\mathrm{E}$ & {$[33]$} \\
\hline \multicolumn{5}{|l|}{ Cucurbitaceae } \\
\hline Benincasa hispida & Sri Lanka & ND & $\mathrm{E}$ & {$[18]$} \\
\hline Citrullus colocynthis ${ }^{*}$ & India, Pakistan & Fruit, root & ND & {$[17,40,41]$} \\
\hline Coccinia grandis & Pakistan, Sri Lanka & Root & I, E & {$[17,18]$} \\
\hline Corallocarpus epigaeus & India & Tuber & I & {$[38]$} \\
\hline Cucumis melo & Sri Lanka & ND & I & {$[18]$} \\
\hline Cucurbita pepo & Spain & Flower & $\mathrm{E}$ & {$[37]$} \\
\hline Diplocyclos palmatus & India, Sri Lanka & Leaf, tuber & $\mathrm{I}, \mathrm{E}$ & {$[18,23,66]$} \\
\hline Fevillea cordifolia & Colombia, Nicaragua & Seed, whole plant & I, E & {$[27,35]$} \\
\hline Lagenaria siceraria ${ }^{\#}$ & Sri Lanka & $\mathrm{ND}$ & $\mathrm{E}$ & {$[18]$} \\
\hline Luffa acutangula & India, Sri Lanka & Fruit, whole plant & I, E & {$[18,19,38]$} \\
\hline Momordica balsamina & India & ND & ND & [40] \\
\hline Momordica charantia* & $\begin{array}{l}\text { Colombia, India, } \\
\text { Nicaragua, Sri Lanka }\end{array}$ & $\begin{array}{l}\text { Aerial parts, branch, flower, fruit, } \\
\text { leaf, stem, whole plant }\end{array}$ & $\mathrm{I}, \mathrm{E}$ & {$[18,20,27,34,35]$} \\
\hline Momordica dioica & Sri Lanka & ND & $\mathrm{E}$ & {$[18]$} \\
\hline Sicydium tamnifolium & Mexico & Root & ND & [59] \\
\hline Trichosanthes cucumerina & India, Sri Lanka & Leaf & I & {$[18,38]$} \\
\hline Trichosanthes tricuspidata & Bangladesh & Root & $\mathrm{I}$ & \\
\hline \multicolumn{5}{|l|}{ Cycadaceae } \\
\hline Cycas pectinata & Bangladesh & Flower & $\mathrm{E}$ & {$[32]$} \\
\hline Cycas revoluta & Bangladesh & Whole plant & ND & {$[30]$} \\
\hline \multicolumn{5}{|l|}{ Cyclanthaceae } \\
\hline Cyclanthus bipartitus & Peru & Heart & $\mathrm{E}$ & {$[57]$} \\
\hline \multicolumn{5}{|l|}{ Cyperaceae } \\
\hline Cyperus kyllingia & Sri Lanka & ND & I & {$[18]$} \\
\hline Cyperus rotundus & $\begin{array}{l}\text { Bangladesh, India, } \\
\text { Pakistan, Sri Lanka }\end{array}$ & $\begin{array}{l}\text { Bulb, flower, leaf, rhizome, root, } \\
\text { tuber }\end{array}$ & $\mathrm{I}, \mathrm{E}$ & {$[17,18,20,28,32,39]$} \\
\hline $\begin{array}{l}\text { Kyllinga odorata (syn. Kyllinga } \\
\text { monocephala) }\end{array}$ & India & 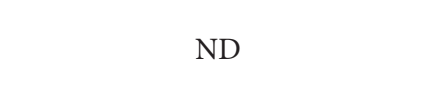 & ND & {$[40]$} \\
\hline \multicolumn{5}{|l|}{ Dilleniaceae } \\
\hline Tetracera sarmentosa & Sri Lanka & ND & $\mathrm{I}, \mathrm{E}$ & {$[18]$} \\
\hline \multicolumn{5}{|l|}{ Dioscoreaceae } \\
\hline Dioscorea oppositifolia & Sri Lanka & ND & I & {$[18]$} \\
\hline Dioscorea pentaphylla & India & Tuber & I & {$[38,55]$} \\
\hline
\end{tabular}


TABle 1: Continued.

\begin{tabular}{|c|c|c|c|c|}
\hline Plant name & Countries & Parts used & Use & Reference(s) \\
\hline \multicolumn{5}{|l|}{ Dipterocarpaceae } \\
\hline Dipterocarpus lowii & Sri Lanka & ND & I & {$[18]$} \\
\hline Dipterocarpus zeylanicus & Sri Lanka & $\mathrm{ND}$ & $\mathrm{E}$ & {$[18]$} \\
\hline \multicolumn{5}{|l|}{ Droseraceae } \\
\hline Drosera burmannii & Sri Lanka & ND & $\mathrm{I}, \mathrm{E}$ & {$[18]$} \\
\hline Drosera indica & Sri Lanka & ND & $\mathrm{E}$ & {$[18]$} \\
\hline \multicolumn{5}{|l|}{ Ebenaceae } \\
\hline Diospyros kaki & Malaysia & Fruit & I & [67] \\
\hline Diospyros melanoxylon & India & Seed & $\mathrm{E}$ & [25] \\
\hline Diospyros montana & India & Root & I & {$[38]$} \\
\hline Diospyros vera (syn. Maba buxifolia) & Sri Lanka & ND & I, E & {$[18]$} \\
\hline Euclea racemosa & Ethiopia & Leaf & I & {$[51]$} \\
\hline \multicolumn{5}{|l|}{ Elaeagnaceae } \\
\hline Elaeagnus latifolia & Sri Lanka & ND & $\mathrm{I}, \mathrm{E}$ & {$[18]$} \\
\hline \multicolumn{5}{|l|}{ Ericaceae } \\
\hline Gaultheria trichophylla & India & Leaf & I & {$[66]$} \\
\hline \multicolumn{5}{|l|}{ Erythroxylaceae } \\
\hline Erythroxylum monogynum & Sri Lanka & ND & $\mathrm{E}$ & {$[18]$} \\
\hline \multicolumn{5}{|l|}{ Euphorbiaceae } \\
\hline $\begin{array}{l}\text { Acalypha aristata (syn. Acalypha } \\
\text { arvensis) }\end{array}$ & Nicaragua & Leaf, whole plant & I, E & [27] \\
\hline Acalypha fimbriata & ND & ND & ND & {$[68]$} \\
\hline Acalypha indica* & $\begin{array}{l}\text { Bangladesh, India, Sri } \\
\text { Lanka }\end{array}$ & Leaf, whole plant & $\mathrm{E}$ & {$[18,20,32]$} \\
\hline Acalypha phleoides & Mexico & ND & ND & {$[68]$} \\
\hline $\begin{array}{l}\text { Acalypha wilkesiana (syn. Acalypha } \\
\text { godseffiana) }\end{array}$ & Sri Lanka & ND & $\mathrm{E}$ & {$[18]$} \\
\hline Agrostistachys hookeri & Sri Lanka & ND & $\mathrm{E}$ & {$[18]$} \\
\hline $\begin{array}{l}\text { Baliospermum solanifolium (syn. } \\
\text { Baliospermum montanum) }\end{array}$ & India & Leaf, root, seed & E & {$[19,32]$} \\
\hline Cnidoscolus aconitifolius & Colombia & Leaf, whole plant & $\mathrm{I}, \mathrm{E}$ & {$[34]$} \\
\hline Croton tiglium & Sri Lanka & ND & $\mathrm{E}$ & {$[18]$} \\
\hline Croton trinitatis & Colombia & Whole plant & $\mathrm{E}$ & {$[34]$} \\
\hline Euphorbia antiquorum & Sri Lanka & ND & $\mathrm{E}$ & {$[18]$} \\
\hline Euphorbia hirta* & Bangladesh, Brazil, India & Latex, root, whole plant & $\mathrm{I}$ & {$[19,20,32,47]$} \\
\hline Euphorbia milii & Bangladesh & Whole plant & ND & {$[30]$} \\
\hline $\begin{array}{l}\text { Euphorbia neriifolia (syn. Euphorbia } \\
\text { ligularia) }\end{array}$ & India, Sri Lanka & Latex, leaf, stem & $\mathrm{I}, \mathrm{E}$ & {$[18,19,22,38]$} \\
\hline Euphorbia thymifolia & Nicaragua & Latex, leaf, whole plant & I & [27] \\
\hline Euphorbia tirucalli & Sri Lanka & ND & I & {$[18]$} \\
\hline $\begin{array}{l}\text { Euphorbia tithymaloides (syn. } \\
\text { Pedilanthus tithymaloides) }\end{array}$ & Sri Lanka & ND & $\mathrm{I}, \mathrm{E}$ & {$[18]$} \\
\hline Euphorbia tortilis & Sri Lanka & ND & $\mathrm{E}$ & {$[18]$} \\
\hline Hura crepitans & Peru & Latex & $\mathrm{E}$ & [57] \\
\hline Jatropha curcas* & Brazil, Nepal & Latex, root, stem & I & {$[47,64,69,70]$} \\
\hline Jatropha gossypiifolia* & Bangladesh, Brazil & Latex, leaf, stem & $\mathrm{I}, \mathrm{E}$ & {$[32,50]$} \\
\hline Jatropha mollissima* & Brazil & Latex & ND & {$[47,64]$} \\
\hline Jatropha multifida & Sri Lanka & $\mathrm{ND}$ & $\mathrm{E}$ & {$[18]$} \\
\hline Jatropha podagrica & Sri Lanka & ND & E & {$[18]$} \\
\hline
\end{tabular}


TABle 1: Continued.

\begin{tabular}{|c|c|c|c|c|}
\hline Plant name & Countries & Parts used & Use & Reference(s) \\
\hline Jatropha ribifolia & Brazil & Latex & ND & [47] \\
\hline Mallotus repandus & Sri Lanka & $\mathrm{ND}$ & $\mathrm{E}$ & {$[18]$} \\
\hline Manihot esculenta & $\begin{array}{l}\text { Brazil, Colombia, } \\
\text { Nicaragua }\end{array}$ & Branch, leaf, root & $\mathrm{I}, \mathrm{E}$ & {$[27,33,34]$} \\
\hline Melanolepis multiglandulosa & Papua New Guinea & Latex & I & {$[46]$} \\
\hline Phyllanthus acuminatus ${ }^{\#}$ & Colombia & Branch, leaf & $\mathrm{I}, \mathrm{E}$ & [35] \\
\hline Ricinus communis & $\begin{array}{l}\text { Brazil, Pakistan, Sri } \\
\text { Lanka }\end{array}$ & Fruit, latex, leaf, root, seed & $\mathrm{I}, \mathrm{E}$ & {$[17,18,69,71,72]$} \\
\hline Spirostachys africana & Namibia & Stem & ND & {$[65]$} \\
\hline Tragia involucrata & India & Whole plant & I & {$[20,28]$} \\
\hline Trewia nudiflora & Bangladesh & Leaf & $\mathrm{E}$ & [32] \\
\hline \multicolumn{5}{|l|}{ Fabaceae } \\
\hline Abrus precatorius* & Bangladesh, India & Leaf, root, stem & $\mathrm{I}, \mathrm{E}$ & {$[20,21,28,32,38]$} \\
\hline Abrus pulchellus & Sri Lanka & ND & $\mathrm{E}$ & {$[18]$} \\
\hline Acacia caesia & Sri Lanka & ND & $\mathrm{I}, \mathrm{E}$ & {$[18]$} \\
\hline Acacia cornigera & Mexico & Root & ND & [59] \\
\hline Acacia leucophloea & India & Bark & $\mathrm{I}, \mathrm{E}$ & {$[20,63]$} \\
\hline Acacia mellifera & Namibia & ND & ND & [54] \\
\hline Acacia nilotica & India & Leaf & $\mathrm{I}, \mathrm{E}$ & {$[38]$} \\
\hline Acacia torta & India & Bark & I & {$[63]$} \\
\hline Acosmium panamense & Mexico & Bark & ND & {$[59]$} \\
\hline Adenanthera pavonina & Sri Lanka & ND & $\mathrm{I}, \mathrm{E}$ & {$[18]$} \\
\hline Albizia lebbeck* & $\begin{array}{l}\text { Bangladesh, India, } \\
\text { Pakistan, Sri Lanka }\end{array}$ & Bark, flower, fruit, leaf, seed & $\mathrm{I}, \mathrm{E}$ & {$[16-18,23,32,40]$} \\
\hline Albizia procera & Bangladesh, Pakistan & Juicy parts, leaf, root & $\mathrm{E}$ & {$[17,32]$} \\
\hline Alysicarpus vaginalis & Sri Lanka & ND & I & {$[18]$} \\
\hline Amburana cearensis & Brazil & Seed & ND & [71] \\
\hline $\begin{array}{l}\text { Bauhinia divaricata (syn. Bauhinia } \\
\text { retusa) }\end{array}$ & India & Bark, flower, leaf & ND & {$[19]$} \\
\hline Bauhinia guianensis & Nicaragua & Bark, stem & $\mathrm{I}, \mathrm{E}$ & {$[27]$} \\
\hline Bauhinia purpurea & India & Bark, flower, leaf & ND & {$[19]$} \\
\hline Bauhinia racemosa & Sri Lanka & ND & $\mathrm{E}$ & {$[18]$} \\
\hline Bauhinia variegata* & Bangladesh, Sri Lanka & Bulb, stem & $\mathrm{E}$ & {$[18,32]$} \\
\hline Brownea rosa-de-monte ${ }^{*}$ & Colombia & Bark & $\mathrm{I}, \mathrm{E}$ & {$[35]$} \\
\hline Butea monosperma* & India & Bark, leaf, resin, seed & $\mathrm{I}, \mathrm{E}$ & {$[24,25,38,40,41]$} \\
\hline Caesalpinia bonduc & $\begin{array}{l}\text { India, Nicaragua, Sri } \\
\text { Lanka }\end{array}$ & Root, seed & $\mathrm{I}, \mathrm{E}$ & {$[18,20,27,38]$} \\
\hline Caesalpinia coriaria & Sri Lanka & ND & $\mathrm{E}$ & {$[18]$} \\
\hline Cajanus cajan & Bangladesh & Stem & $\mathrm{E}$ & {$[30,32]$} \\
\hline Canavalia gladiata & Sri Lanka & ND & $\mathrm{E}$ & {$[18]$} \\
\hline Cassia fistula* & $\begin{array}{l}\text { Bangladesh, Brazil, } \\
\text { India, Sri Lanka }\end{array}$ & Bark, fruit, leaf, root, seed & $\mathrm{I}, \mathrm{E}$ & $\begin{array}{c}{[18,19,24,25,32,33,} \\
38,40]\end{array}$ \\
\hline Centrosema pubescens & Colombia & Whole plant & $\mathrm{E}$ & {$[34]$} \\
\hline Clitoria ternatea & $\begin{array}{l}\text { Bangladesh, India, Sri } \\
\text { Lanka }\end{array}$ & Flower, leaf, root, seed & $\mathrm{I}, \mathrm{E}$ & $\begin{array}{c}{[16,18,19,32,38,39,} \\
42,60]\end{array}$ \\
\hline Crotalaria laburnifolia & Sri Lanka & ND & $\mathrm{E}$ & {$[18]$} \\
\hline Crotalaria verrucosa & India & Seed & I & [23] \\
\hline Dalbergia melanoxylon & India & Bark & I & {$[20]$} \\
\hline $\begin{array}{l}\text { Deguelia amazonica (syn. Derris } \\
\text { amazonica) }\end{array}$ & Brazil & Root & ND & {$[50]$} \\
\hline
\end{tabular}


TABle 1: Continued.

\begin{tabular}{|c|c|c|c|c|}
\hline Plant name & Countries & Parts used & Use & Reference(s) \\
\hline Derris floribunda & Brazil & Root & ND & {$[50]$} \\
\hline Desmodium adscendens ${ }^{\#}$ & Colombia, Nicaragua & Leaf, root, whole plant & $\mathrm{I}, \mathrm{E}$ & {$[27,35]$} \\
\hline Desmodium gangeticum & $\begin{array}{l}\text { Bangladesh, India, } \\
\text { Pakistan }\end{array}$ & Root, whole plant & $\mathrm{I}, \mathrm{E}$ & {$[17,32,55]$} \\
\hline Desmodium triflorum & Bangladesh, Sri Lanka & Shoot & $\mathrm{I}, \mathrm{E}$ & {$[18,32]$} \\
\hline Dipteryx odorata & Brazil & Seed & I & {$[33,50]$} \\
\hline Entada leptostachya & Kenya & Latex & $\mathrm{E}$ & [31] \\
\hline $\begin{array}{l}\text { Entada rheedii (syn. Entada } \\
\text { pursaetha) }\end{array}$ & $\begin{array}{c}\text { Bangladesh, India, Sri } \\
\text { Lanka }\end{array}$ & Leaf, seed & I, E & {$[18,32,49]$} \\
\hline Erythrina americana & Mexico & Leaf, seed & ND & [59] \\
\hline Erythrina excelsa & India, Kenya & Bark, latex & ND & {$[20,31]$} \\
\hline Erythrina fusca & Sri Lanka & ND & I, E & [18] \\
\hline Erythrina subumbrans & Sri Lanka & ND & I & {$[18]$} \\
\hline Erythrina variegata & India & Bark & ND & {$[19]$} \\
\hline Gliricidia sepium & Colombia & Leaf, stem & $\mathrm{I}, \mathrm{E}$ & {$[34]$} \\
\hline Glycine max & India & Seed & $\mathrm{I}$ & {$[20]$} \\
\hline Glycyrrhiza glabra & Sri Lanka & ND & $\mathrm{E}$ & [18] \\
\hline Humboldtia decurrens & India & Root & $\mathrm{E}$ & [16] \\
\hline Humboldtia laurifolia & Sri Lanka & ND & $\mathrm{E}$ & {$[18]$} \\
\hline Indigofera circinella & Kenya & Leaf & $\mathrm{E}$ & {$[31]$} \\
\hline Indigofera suffruticosa & Colombia, Nicaragua & Aerial parts, seed, whole plant & I, E & {$[27,34]$} \\
\hline Indigofera tinctoria $a^{\#}$ & India & Root & I & {$[16]$} \\
\hline Leucaena leucocephala & Sri Lanka & ND & $\mathrm{E}$ & {$[18]$} \\
\hline Libidibia ferrea & Brazil & Seed & $\mathrm{I}$ & {$[33]$} \\
\hline Machaerium ferox & Brazil & Leaf & $\mathrm{E}$ & {$[33]$} \\
\hline Macrotyloma uniflorum & Sri Lanka & ND & I & {$[18]$} \\
\hline Mimosa pudica* & Bangladesh, India & Leaf, root, whole plant & $\mathrm{I}, \mathrm{E}$ & $\begin{array}{c}{[16,19,20,22,23,28,} \\
32]\end{array}$ \\
\hline Mucuna pruriens & $\begin{array}{l}\text { Bangladesh, India, } \\
\text { Nepal, Sri Lanka }\end{array}$ & Fruit, seed, stem, whole plant & I, E & {$[18,19,28,32,69]$} \\
\hline Mucuna sloanei & Ecuador & Seed & I & {$[61]$} \\
\hline Mucuna urens & Nicaragua & Seed & $\mathrm{E}$ & [27] \\
\hline Parkinsonia aculeata & Brazil & Seed & ND & {$[47]$} \\
\hline Pentaclethra macroloba* & Nicaragua & Bark & I, E & [27] \\
\hline Plathymenia reticulata ${ }^{*}$ & Brazil & Bark & I & {$[33]$} \\
\hline Pongamia pinnata & Sri Lanka & ND & $\mathrm{I}, \mathrm{E}$ & {$[18]$} \\
\hline Pterocarpus santalinus & Sri Lanka & ND & $\mathrm{E}$ & {$[18]$} \\
\hline Saraca asoca & Sri Lanka & ND & I & {$[18]$} \\
\hline Senna alata (syn. Cassia alata) & $\begin{array}{l}\text { India, Nicaragua, Sri } \\
\text { Lanka }\end{array}$ & Flower, leaf, whole plant & $\mathrm{I}, \mathrm{E}$ & {$[18,20,27,28]$} \\
\hline Senna auriculata* & Sri Lanka & ND & $\mathrm{E}$ & {$[18]$} \\
\hline Senna dariensis ${ }^{*}$ & Colombia & Whole plant & $\mathrm{I}, \mathrm{E}$ & {$[35]$} \\
\hline Senna hirsuta & Bangladesh & Leaf & $\mathrm{E}$ & [32] \\
\hline $\begin{array}{l}\text { Senna occidentalis (syn. Cassia } \\
\text { occidentalis) }\end{array}$ & $\begin{array}{l}\text { Bangladesh, India, } \\
\text { Nicaragua, Sri Lanka }\end{array}$ & Leaf, root, whole plant & $\mathrm{I}, \mathrm{E}$ & {$[18,27,32,40]$} \\
\hline $\begin{array}{l}\text { Senna reticulata (syn. Cassia } \\
\text { reticulata) }\end{array}$ & Brazil, Nicaragua & Leaf, root, whole plant & I & {$[27,50]$} \\
\hline Senna siamea & Kenya & Root & ND & {$[31]$} \\
\hline Senna sophera (syn. Cassia sophera) & Bangladesh & Leaf, root & $\mathrm{I}$ & {$[30,32]$} \\
\hline Senna tora (syn. Cassia tora) & Bangladesh, India & Leaf, root, seed, stem & $\mathrm{I}, \mathrm{E}$ & {$[20,24,25,28,32,42]$} \\
\hline
\end{tabular}


TABle 1: Continued.

\begin{tabular}{|c|c|c|c|c|}
\hline Plant name & Countries & Parts used & Use & Reference(s) \\
\hline Sesbania grandiflora & Sri Lanka & ND & $\mathrm{I}, \mathrm{E}$ & {$[18]$} \\
\hline $\begin{array}{l}\text { Tadehagi triquetrum (syn. } \\
\text { Desmodium triquetrum) }\end{array}$ & India & Whole plant & ND & {$[19]$} \\
\hline Tamarindus indica* & $\begin{array}{c}\text { Bangladesh, India, Sri } \\
\text { Lanka }\end{array}$ & Seed, whole plant & $\mathrm{I}, \mathrm{E}$ & {$[18,22,25,32,38$} \\
\hline Tephrosia purpurea & Bangladesh, India & Root, whole plant & $\mathrm{I}, \mathrm{E}$ & {$[19,20,24,32]$} \\
\hline Trigonella foenum-graecum & Sri Lanka & ND & I & {$[18]$} \\
\hline Uraria lagopodioides & India & Bark & $\mathrm{I}, \mathrm{E}$ & [49] \\
\hline Uraria picta & Bangladesh, India & Root, whole plant & I & {$[24,30]$} \\
\hline Vigna luteola & Colombia & Whole plant & $\mathrm{E}$ & {$[34]$} \\
\hline Vigna radiata & Sri Lanka & ND & I & {$[18]$} \\
\hline \multicolumn{5}{|l|}{ Gentianaceae } \\
\hline $\begin{array}{l}\text { Chelonanthus alatus (syn. Irlbachia } \\
\text { alata) }\end{array}$ & Colombia & Branch, leaf & $\mathrm{E}$ & {$[35]$} \\
\hline Enicostema axillare ${ }^{*}$ & India & Whole plant & I & {$[23,45]$} \\
\hline Fagraea ceilanica & Sri Lanka & ND & $\mathrm{E}$ & [18] \\
\hline Hoppea dichotoma & India & Shoot & ND & [19] \\
\hline Huperzia phlegmaria & Sri Lanka & ND & $\mathrm{E}$ & {$[18]$} \\
\hline Potalia amara & Peru & Aerial parts & ND & {$[29]$} \\
\hline \multicolumn{5}{|l|}{ Gesneriaceae } \\
\hline Columnea pulcherrima & Colombia & Whole plant & $\mathrm{I}, \mathrm{E}$ & {$[35]$} \\
\hline $\begin{array}{l}\text { Columnea sanguinea (syn. Besleria } \\
\text { sanguinea) }\end{array}$ & Colombia & Whole plant & $\mathrm{I}, \mathrm{E}$ & {$[35]$} \\
\hline Episcia dianthiflora & Colombia & Whole plant & $\mathrm{I}, \mathrm{E}$ & {$[35]$} \\
\hline \multicolumn{5}{|l|}{ Gleicheniaceae } \\
\hline Gleichenella pectinata & Colombia & Whole plant & I & {$[34]$} \\
\hline \multicolumn{5}{|l|}{ Haemodoraceae } \\
\hline Xiphidium caeruleum ${ }^{\#}$ & $\begin{array}{c}\text { Colombia, Nicaragua, } \\
\text { Peru }\end{array}$ & Leaf, stem, whole plant & $\mathrm{I}, \mathrm{E}$ & {$[27,35,44,57]$} \\
\hline \multicolumn{5}{|l|}{ Heliconiaceae } \\
\hline Heliconia curtispatha* & Colombia & Rhizome & $\mathrm{E}$ & {$[35]$} \\
\hline \multicolumn{5}{|l|}{ Hydroleaceae } \\
\hline Hydrolea zeylanica & Sri Lanka & ND & $\mathrm{I}$ & {$[18]$} \\
\hline \multicolumn{5}{|l|}{ Hymenophyllaceae } \\
\hline Trichomanes elegans ${ }^{*}$ & Colombia & Whole plant & $\mathrm{E}$ & {$[35]$} \\
\hline \multicolumn{5}{|l|}{ Hypoxidaceae } \\
\hline Curculigo orchioides & Bangladesh, India & Bulb, leaf, rhizome & I & {$[32,73]$} \\
\hline \multicolumn{5}{|l|}{ Iridaceae } \\
\hline Iris kemaonensis & India & Rhizome & ND & {$[66]$} \\
\hline Sisyrinchium micranthum & Colombia & Whole plant & $\mathrm{E}$ & {$[34]$} \\
\hline \multicolumn{5}{|l|}{ Lamiaceae } \\
\hline Aegiphila panamensis ${ }^{\#}$ & Colombia & Leaf, branch, stem & $\mathrm{E}$ & {$[35]$} \\
\hline Anisochilus velutinus & Sri Lanka & ND & $\mathrm{E}$ & {$[18]$} \\
\hline Anisomeles indica & India, Sri Lanka & Whole plant & ND & {$[18,19]$} \\
\hline Anisomeles malabarica & Bangladesh, India & Whole plant & I & {$[28,30,60]$} \\
\hline Callicarpa tomentosa & Sri Lanka & ND & $\mathrm{E}$ & [18] \\
\hline $\begin{array}{l}\text { Clerodendrum cordatum (syn. } \\
\text { Clerodendrum viscosum) }\end{array}$ & Bangladesh & Flower, leaf & $\mathrm{E}$ & {$[32]$} \\
\hline Clerodendrum phlomidis & Sri Lanka & ND & $\mathrm{E}$ & {$[18]$} \\
\hline
\end{tabular}


TABle 1: Continued.

\begin{tabular}{|c|c|c|c|c|}
\hline Plant name & Countries & Parts used & Use & Reference(s) \\
\hline Fuerstia africana & Kenya & Leaf & I & {$[31]$} \\
\hline Gmelina arborea & Bangladesh & Root & I & [32] \\
\hline Gmelina asiatica & Sri Lanka & ND & $\mathrm{I}, \mathrm{E}$ & {$[18]$} \\
\hline Hyptis capitata & Colombia & Branch, leaf, stem & $\mathrm{I}, \mathrm{E}$ & {$[35]$} \\
\hline Hyptis suaveolens & Bangladesh & Leaf & $\mathrm{E}$ & [32] \\
\hline Leonotis leonurus & South Africa & Flower, leaf & I & {$[74]$} \\
\hline Leucas aspera ${ }^{*}$ & Bangladesh, India & Leaf, root, stem & I & {$[23,24,28,30,32]$} \\
\hline Leucas cephalotes ${ }^{*}$ & India & Bark, leaf, whole plant & $\mathrm{I}, \mathrm{E}$ & {$[19,20,40,49]$} \\
\hline Marsypianthes chamaedrys* & Brazil & Leaf & I & [33] \\
\hline Mentha $\times$ piperita & Colombia & Leaf & $\mathrm{E}$ & [34] \\
\hline Mentha pulegium & Colombia & Leaf & $\mathrm{E}$ & [34] \\
\hline Ocimum basilicum ${ }^{\#}$ & $\begin{array}{l}\text { Bangladesh, Colombia, } \\
\text { India }\end{array}$ & Branch, leaf, stem, whole plant & $\mathrm{I}, \mathrm{E}$ & {$[20,32,35]$} \\
\hline $\begin{array}{l}\text { Ocimum campechianum (syn. } \\
\text { Ocimum micranthum) }\end{array}$ & Colombia, Nicaragua & Aerial parts, leaf, whole plant & I, E & {$[27,44]$} \\
\hline $\begin{array}{l}\text { Ocimum tenuiflorum (syn. Ocimum } \\
\text { sanctum) }\end{array}$ & India, Sri Lanka & Leaf, root, whole plant & $\mathrm{I}, \mathrm{E}$ & {$[16,18,20,28,40,41]$} \\
\hline Origanum vulgare & Serbia & Flower, leaf & ND & {$[58]$} \\
\hline Plectranthus amboinicus & Sri Lanka & ND & I & {$[18]$} \\
\hline Plectranthus hadiensis & Sri Lanka & ND & I & {$[18]$} \\
\hline Plectranthus monostachyus & Brazil & Leaf & I & {$[33]$} \\
\hline Pogostemon cablin & Malaysia & ND & ND & [75] \\
\hline Pogostemon heyneanus & Sri Lanka & ND & $\mathrm{E}$ & {$[18]$} \\
\hline Premna esculenta & Bangladesh & Leaf & $\mathrm{E}$ & {$[32]$} \\
\hline $\begin{array}{l}\text { Premna serratifolia (syn. Premna } \\
\text { integrifolia) }\end{array}$ & Bangladesh & Leaf, root & I, E & {$[36]$} \\
\hline Rosmarinus officinalis & Colombia & Whole plant & $\mathrm{E}$ & [34] \\
\hline $\begin{array}{l}\text { Rotheca serrata (syn. Clerodendrum } \\
\text { serratum) }\end{array}$ & India & Leaf, root & ND & {$[19,39]$} \\
\hline Tectona grandis & India & Bark & I & {$[25]$} \\
\hline Teucrium chamaedrys & Serbia & Flower & ND & [58] \\
\hline Thymus vulgaris & India, Spain & Aerial parts, whole plant & $\mathrm{I}, \mathrm{E}$ & {$[20,37]$} \\
\hline Vitex negundo $^{*}$ & $\begin{array}{l}\text { Bangladesh, India, Sri } \\
\text { Lanka }\end{array}$ & Leaf, rhizome, root & I, E & {$[18,20,22,32]$} \\
\hline Vitex trifolia & India & Leaf & I & {$[28]$} \\
\hline $\begin{array}{l}\text { Volkameria eriophylla (syn. } \\
\text { Clerodendrum eriophyllum) }\end{array}$ & Kenya & Leaf, root & ND & {$[76]$} \\
\hline \multicolumn{5}{|l|}{ Lauraceae } \\
\hline $\begin{array}{l}\text { Aniba parviflora (syn. Aniba } \\
\text { fragrans) }\end{array}$ & Brazil & Bark & I & [33] \\
\hline Cinnamomum verum & Sri Lanka & ND & I, E & {$[18]$} \\
\hline Litsea glutinosa & Sri Lanka & ND & $\mathrm{E}$ & {$[18]$} \\
\hline Litsea longifolia & Sri Lanka & ND & I, E & {$[18]$} \\
\hline Persea macrantha & Sri Lanka & ND & $\mathrm{E}$ & {$[18]$} \\
\hline \multicolumn{5}{|l|}{ Lecythidaceae } \\
\hline Careya arborea & Sri Lanka & ND & $\mathrm{E}$ & {$[18]$} \\
\hline Couroupita guianensis & Bangladesh & Bark, leaf & ND & {$[30]$} \\
\hline \multicolumn{5}{|l|}{ Linderniaceae } \\
\hline Lindernia diffusa ${ }^{\#}$ & Colombia & Whole plant & $\mathrm{E}$ & [35] \\
\hline
\end{tabular}


TABle 1: Continued.

\begin{tabular}{|c|c|c|c|c|}
\hline Plant name & Countries & Parts used & Use & Reference(s) \\
\hline \multicolumn{5}{|l|}{ Loganiaceae } \\
\hline Strychnos nux-vomica* & India & Bark, root, seed & $\mathrm{I}, \mathrm{E}$ & {$[16,20,49]$} \\
\hline Strychnos potatorum & Sri Lanka & ND & $\mathrm{E}$ & [18] \\
\hline Strychnos xinguensis* & Colombia & Stem & $\mathrm{E}$ & {$[35]$} \\
\hline \multicolumn{5}{|l|}{ Loranthaceae } \\
\hline Struthanthus cassythoides & Nicaragua & Leaf, whole plant & $\mathrm{I}, \mathrm{E}$ & {$[27]$} \\
\hline Struthanthus orbicularis* & Colombia & Branch, leaf & $\mathrm{E}$ & {$[35]$} \\
\hline \multicolumn{5}{|l|}{ Lycopodiaceae } \\
\hline Huperzia pulcherrima & Sri Lanka & ND & $\mathrm{E}$ & {$[18]$} \\
\hline \multicolumn{5}{|l|}{ Lygodiaceae } \\
\hline Lygodium heterodoxum & Nicaragua & Leaf & I, E & {$[27]$} \\
\hline Lygodium venustum & $\begin{array}{l}\text { Colombia, Mexico, } \\
\text { Nicaragua }\end{array}$ & $\begin{array}{l}\text { Aerial parts, leaf, stem, whole } \\
\text { plant }\end{array}$ & I, E & {$[27,34,59]$} \\
\hline \multicolumn{5}{|l|}{ Lythraceae } \\
\hline Lawsonia inermis & India & Bark & ND & {$[25]$} \\
\hline Punica granatum & India, Sri Lanka & Whole plant & I, E & {$[18,20,28]$} \\
\hline Trapa natans (syn. Trapa bispinosa) & Sri Lanka & ND & I & [18] \\
\hline \multicolumn{5}{|l|}{ Magnoliaceae } \\
\hline $\begin{array}{l}\text { Magnolia champaca (syn. Michelia } \\
\text { champaca) }\end{array}$ & Sri Lanka & ND & $\mathrm{E}$ & {$[18]$} \\
\hline \multicolumn{5}{|l|}{ Malpighiaceae } \\
\hline $\begin{array}{l}\text { Bronwenia cornifolia (syn. } \\
\text { Banisteriopsis cornifolia) }\end{array}$ & Nicaragua & Bark, leaf, stem & $\mathrm{E}$ & {$[27]$} \\
\hline Byrsonima crassifolia & Brazil, Nicaragua & Bark, leaf & I & {$[27,47]$} \\
\hline Stigmaphyllon puberum & Nicaragua & Leaf, stem & $\mathrm{I}, \mathrm{E}$ & [27] \\
\hline \multicolumn{5}{|l|}{ Malvaceae } \\
\hline Abelmoschus moschatus & $\begin{array}{l}\text { Bangladesh, India, Sri } \\
\text { Lanka }\end{array}$ & Fruit, leaf, seed & $\mathrm{I}, \mathrm{E}$ & {$[18,32,38]$} \\
\hline Abroma augusta & Bangladesh & Leaf, root, stem & $\mathrm{E}$ & {$[32]$} \\
\hline $\begin{array}{l}\text { Abutilon hirtum (syn. Abutilon } \\
\text { heterotrichum) }\end{array}$ & Sri Lanka & ND & I, E & {$[18]$} \\
\hline Abutilon indicum & India, Sri Lanka & Fruit, leaf & I & {$[18,20]$} \\
\hline Ceiba pentandra & Sri Lanka & ND & I & {$[18]$} \\
\hline Corchorus trilocularis & Kenya & Leaf & $\mathrm{E}$ & {$[31]$} \\
\hline $\begin{array}{l}\text { Firmiana simplex (syn. Sterculia } \\
\text { urens) }\end{array}$ & India & Bark, latex & I & {$[38,55]$} \\
\hline Gossypium arboreum & Sri Lanka & ND & $\mathrm{E}$ & {$[18]$} \\
\hline Gossypium herbaceum & India & Seed & ND & {$[41]$} \\
\hline Gossypium hirsutum & Brazil & Leaf & I & {$[33]$} \\
\hline Grewia damine & Sri Lanka & ND & $\mathrm{E}$ & {$[18]$} \\
\hline $\begin{array}{l}\text { Grewia nervosa (syn. Microcos } \\
\text { paniculata) }\end{array}$ & Sri Lanka & ND & $\mathrm{E}$ & {$[18]$} \\
\hline Helicteres isora & Bangladesh, India & Fruit, root & I & {$[23,25,32]$} \\
\hline $\begin{array}{l}\text { Hibiscus rostellatus (syn. Hibiscus } \\
\text { furcatus) }\end{array}$ & Sri Lanka & ND & $\mathrm{E}$ & {$[18]$} \\
\hline Hibiscus surattensis & Sri Lanka & ND & $\mathrm{E}$ & {$[18]$} \\
\hline Hibiscus tiliaceus & Mexico & Seed & ND & [59] \\
\hline Melochia corchorifolia & Bangladesh, Sri Lanka & Leaf, whole plant & I, E & {$[18,32]$} \\
\hline Sida acuta $a^{\#}$ & $\begin{array}{l}\text { Bangladesh, Colombia, } \\
\text { India, Sri Lanka }\end{array}$ & Leaf, whole plant & I, E & {$[18,32,35,39,44]$} \\
\hline
\end{tabular}


TABLe 1: Continued.

\begin{tabular}{|c|c|c|c|c|}
\hline Plant name & Countries & Parts used & Use & Reference(s) \\
\hline Sida cordata & Sri Lanka & ND & I & {$[18]$} \\
\hline Sida cordifolia & Bangladesh & Leaf & I & {$[32]$} \\
\hline Sida rhombifolia & $\begin{array}{l}\text { Bangladesh, Nicaragua, } \\
\text { Sri Lanka }\end{array}$ & Leaf, stem & $\mathrm{I}, \mathrm{E}$ & {$[18,27,32]$} \\
\hline Thespesia populnea & Sri Lanka & ND & I & {$[18]$} \\
\hline Triumfetta rhomboidea & Kenya & Root & $\mathrm{E}$ & {$[31]$} \\
\hline Urena lobata & Bangladesh & Root & I & [32] \\
\hline Wissadula periplocifolia & Bangladesh, Sri Lanka & Leaf, root & $\mathrm{E}$ & {$[18,30,60]$} \\
\hline \multicolumn{5}{|l|}{ Marantaceae } \\
\hline Ischnosiphon rotundifolius & Brazil & Leaf & ND & {$[47]$} \\
\hline \multicolumn{5}{|l|}{ Martyniaceae } \\
\hline Martynia annua & India, Sri Lanka & Fruit & $\mathrm{E}$ & {$[18,25]$} \\
\hline \multicolumn{5}{|l|}{ Melastomataceae } \\
\hline Osbeckia octandra & Sri Lanka & ND & $\mathrm{E}$ & {$[18]$} \\
\hline Bellucia dichotoma* & Brazil & Bark & I & {$[33]$} \\
\hline Melastoma malabathricum & Bangladesh & Leaf & $\mathrm{E}$ & {$[32]$} \\
\hline Memecylon umbellatum & India & Leaf & I & {$[63]$} \\
\hline \multicolumn{5}{|l|}{ Meliaceae } \\
\hline Azadirachta indica & India, Sri Lanka & Bark, flower, latex, leaf, seed & $\mathrm{I}, \mathrm{E}$ & {$[18,20,22,28,39-41]$} \\
\hline Cipadessa baccifera & India & Leaf, root & I & {$[63]$} \\
\hline Melia azedarach & India, Sri Lanka & Bark, leaf & $\mathrm{I}, \mathrm{E}$ & {$[18,41]$} \\
\hline Munronia pinnata & Sri Lanka & ND & $\mathrm{I}, \mathrm{E}$ & [18] \\
\hline \multicolumn{5}{|l|}{ Menispermaceae } \\
\hline Cissampelos fasciculata & Colombia & Leaf & I & {$[44]$} \\
\hline Cissampelos pareira* & $\begin{array}{l}\text { Bangladesh, India, } \\
\text { Mexico, Nicaragua, Sri } \\
\text { Lanka }\end{array}$ & Leaf, root, whole plant & $\mathrm{I}, \mathrm{E}$ & $\begin{array}{c}{[18,19,23,25,27,32,} \\
38,55,59]\end{array}$ \\
\hline Cocculus acuminatus & India & Stem & $\mathrm{E}$ & {$[16]$} \\
\hline $\begin{array}{l}\text { Cocculus hirsutus (syn. Cocculus } \\
\text { villosus) }\end{array}$ & India & Leaf & I & {$[38,40]$} \\
\hline Coscinium fenestratum & Sri Lanka & ND & I & {$[18]$} \\
\hline Cyclea peltata & Sri Lanka & ND & $\mathrm{I}$ & {$[18]$} \\
\hline Odontocarya tenacissima ${ }^{\#}$ & Colombia & Whole plant & $\mathrm{I}, \mathrm{E}$ & {$[35]$} \\
\hline Tinospora cordifolia & $\begin{array}{l}\text { Bangladesh, India, Sri } \\
\text { Lanka }\end{array}$ & Fruit, root, stem & I & {$[18,22,23,32]$} \\
\hline \multicolumn{5}{|l|}{ Menyanthaceae } \\
\hline Nymphoides indica & Nicaragua, Sri Lanka & Leaf, root & I, E & {$[18,27]$} \\
\hline \multicolumn{5}{|l|}{ Monimiaceae } \\
\hline Hortonia angustifolia & Sri Lanka & $\mathrm{ND}$ & $\mathrm{E}$ & {$[18]$} \\
\hline \multicolumn{5}{|l|}{ Moraceae } \\
\hline Artocarpus heterophyllus & Sri Lanka & ND & $\mathrm{E}$ & {$[18]$} \\
\hline Artocarpus nobilis & Sri Lanka & ND & $\mathrm{I}, \mathrm{E}$ & {$[18]$} \\
\hline Broussonetia zeylanica & Sri Lanka & ND & $\mathrm{I}, \mathrm{E}$ & {$[18]$} \\
\hline Castilla elastica* $^{*}$ & Colombia & Branch, leaf, stem & $\mathrm{I}, \mathrm{E}$ & [35] \\
\hline Dorstenia contrajerva & Mexico, Nicaragua & Leaf, whole plant & $\mathrm{I}, \mathrm{E}$ & {$[27,59]$} \\
\hline Ficus benghalensis & India & ND & ND & [40] \\
\hline Ficus drupacea & Sri Lanka & ND & $\mathrm{E}$ & {$[18]$} \\
\hline Ficus hispida & Sri Lanka & ND & $\mathrm{E}$ & {$[18]$} \\
\hline Ficus nymphaeifolia* & Colombia & Branch, leaf, stem & $\mathrm{I}, \mathrm{E}$ & {$[35]$} \\
\hline
\end{tabular}


TABle 1: Continued.

\begin{tabular}{|c|c|c|c|c|}
\hline Plant name & Countries & Parts used & Use & Reference(s) \\
\hline Ficus racemosa & $\begin{array}{l}\text { Bangladesh, India, Sri } \\
\text { Lanka }\end{array}$ & Bark, shoot & $\mathrm{I}, \mathrm{E}$ & {$[18,32,38]$} \\
\hline Ficus religiosa & India, Sri Lanka & Bark & $\mathrm{I}, \mathrm{E}$ & {$[18,49]$} \\
\hline Morus alba* & India & Leaf & I & {$[20]$} \\
\hline Plecospermum spinosum & Sri Lanka & ND & $\mathrm{I}, \mathrm{E}$ & {$[18]$} \\
\hline Streblus asper & Bangladesh & Root & $\mathrm{E}$ & {$[32]$} \\
\hline \multicolumn{5}{|l|}{ Moringaceae } \\
\hline Moringa oleifera ${ }^{\#}$ & India, Sri Lanka & Bark, root, seed & $\mathrm{I}, \mathrm{E}$ & {$[16,18,20,22,24,28]$} \\
\hline \multicolumn{5}{|l|}{ Musaceae } \\
\hline $\begin{array}{l}\text { Ensete ventricosum (syn. Ensete } \\
\text { edule) }\end{array}$ & Kenya & Latex & $\mathrm{E}$ & {$[31]$} \\
\hline Musa $\times$ paradisíaca* & $\begin{array}{c}\text { Ecuador, India, } \\
\text { Nicaragua, Sri Lanka }\end{array}$ & Bark, flower, latex & $\mathrm{I}, \mathrm{E}$ & {$[18,20,27,28,61]$} \\
\hline \multicolumn{5}{|l|}{ Myristicaceae } \\
\hline Myristica fragrans & Sri Lanka & ND & I & {$[18]$} \\
\hline \multicolumn{5}{|l|}{ Myrtaceae } \\
\hline $\begin{array}{l}\text { Myrcia bracteata (syn. Eugenia } \\
\text { bracteata) }\end{array}$ & Sri Lanka & ND & $\mathrm{I}, \mathrm{E}$ & {$[18]$} \\
\hline Syzygium aromaticum & Sri Lanka & ND & I & {$[18]$} \\
\hline Syzygium caryophyllatum & Sri Lanka & ND & $\mathrm{E}$ & {$[18]$} \\
\hline $\begin{array}{l}\text { Syzygium cumini (syn. Eugenia } \\
\text { jambolana) }\end{array}$ & $\begin{array}{l}\text { India, Pakistan, Sri } \\
\text { Lanka }\end{array}$ & Bark, leaf & I & {$[17,18,20]$} \\
\hline Syzygium zeylanicum & Sri Lanka & ND & $\mathrm{E}$ & {$[18]$} \\
\hline \multicolumn{5}{|l|}{ Nelumbonaceae } \\
\hline Nelumbo nucifera & Sri Lanka & ND & I & {$[18]$} \\
\hline \multicolumn{5}{|l|}{ Nepenthaceae } \\
\hline Nepenthes distillatoria & Sri Lanka & ND & $\mathrm{E}$ & {$[18]$} \\
\hline \multicolumn{5}{|l|}{ Nyctaginaceae } \\
\hline Boerhavia coccinea & Pakistan & Whole plant & $\mathrm{E}$ & {$[17]$} \\
\hline Boerhavia diffusa & Brazil, India, Sri Lanka & Leaf, root, whole plant & $\mathrm{E}$ & {$[18,24,25,39,41,50]$} \\
\hline Boerhavia procumbens & Pakistan & Leaf & $\mathrm{E}$ & {$[17]$} \\
\hline Mirabilis jalapa & Bangladesh, Sri Lanka & Leaf & $\mathrm{I}, \mathrm{E}$ & {$[18,32]$} \\
\hline \multicolumn{5}{|l|}{ Nymphaeaceae } \\
\hline Nymphaea nouchali & Sri Lanka & ND & $\mathrm{E}$ & {$[18]$} \\
\hline Nymphaea pubescens & Sri Lanka & ND & I & {$[18]$} \\
\hline \multicolumn{5}{|l|}{ Ochnaceae } \\
\hline Ochna jabotapita & Sri Lanka & ND & I & {$[18]$} \\
\hline Sauvagesia erecta & Nicaragua & Whole plant & $\mathrm{I}, \mathrm{E}$ & {$[27]$} \\
\hline \multicolumn{5}{|l|}{ Oleaceae } \\
\hline Jasminum officinale & Sri Lanka & $\mathrm{ND}$ & $\mathrm{E}$ & {$[18]$} \\
\hline Jasminum sambac & Sri Lanka & ND & $\mathrm{E}$ & [18] \\
\hline Nyctanthes arbor-tristis & India, Sri Lanka & Root & I & {$[18,49]$} \\
\hline Olea europaea & Spain & Oil & ND & [37] \\
\hline \multicolumn{5}{|l|}{ Opiliaceae } \\
\hline Opilia amentacea & Kenya & Root & $\mathrm{E}$ & {$[31]$} \\
\hline \multicolumn{5}{|l|}{ Orchidaceae } \\
\hline Vanda tessellata & India & Root & $\mathrm{E}$ & [25] \\
\hline Zeuxine regia & Sri Lanka & ND & $\mathrm{E}$ & {$[18]$} \\
\hline
\end{tabular}


TABLE 1: Continued.

\begin{tabular}{|c|c|c|c|c|}
\hline Plant name & Countries & Parts used & Use & Reference(s) \\
\hline \multicolumn{5}{|l|}{ Oxalidaceae } \\
\hline Averrhoa carambola & Sri Lanka & ND & I & {$[18]$} \\
\hline Biophytum reinwardtii & Sri Lanka & ND & I & [18] \\
\hline Oxalis corniculata & Bangladesh, Sri Lanka & Leaf & $\mathrm{I}, \mathrm{E}$ & {$[18,32]$} \\
\hline \multicolumn{5}{|l|}{ Pandanaceae } \\
\hline Pandanus kaida & Sri Lanka & ND & I & {$[18]$} \\
\hline $\begin{array}{l}\text { Pandanus odorifer (syn. Pandanus } \\
\text { odoratissimus) }\end{array}$ & India & Root & $\mathrm{ND}$ & {$[19]$} \\
\hline \multicolumn{5}{|l|}{ Papaveraceae } \\
\hline Argemone mexicana & Bangladesh, India & Leaf, root, seed, stem & $\mathrm{I}, \mathrm{E}$ & {$[20,32,38,42]$} \\
\hline \multicolumn{5}{|l|}{ Papilionaceae } \\
\hline Desmodium elegans & Pakistan & Root & $\mathrm{E}$ & {$[17,53]$} \\
\hline \multicolumn{5}{|l|}{ Passifloraceae } \\
\hline Adenia hondala & Sri Lanka & ND & $\mathrm{E}$ & [18] \\
\hline Passiflora quadrangularis* & Colombia & Branch, leaf & $\mathrm{E}$ & {$[34,35]$} \\
\hline \multicolumn{5}{|l|}{ Phyllanthaceae } \\
\hline Antidesma bunius & India & Leaf & ND & {$[77]$} \\
\hline Bridelia retusa & Sri Lanka & ND & $\mathrm{I}, \mathrm{E}$ & {$[18]$} \\
\hline Cleistanthus collinus & Sri Lanka & ND & I & {$[18]$} \\
\hline Glochidion zeylanicum & Sri Lanka & ND & $\mathrm{I}$ & {$[18]$} \\
\hline Margaritaria indica & Sri Lanka & ND & $\mathrm{I}, \mathrm{E}$ & {$[18]$} \\
\hline Phyllanthus acidus & India & Root & ND & {$[77]$} \\
\hline Phyllanthus debilis & Sri Lanka & ND & I & {$[18]$} \\
\hline $\begin{array}{l}\text { Phyllanthus emblica (syn. Emblica } \\
\text { officinalis) }\end{array}$ & $\begin{array}{l}\text { Bangladesh, India, Sri } \\
\text { Lanka }\end{array}$ & Bark, fruit, root & I, E & {$[18,20,22,30]$} \\
\hline Phyllanthus niruri & India & Flower & $\mathrm{E}$ & {$[20]$} \\
\hline Phyllanthus reticulatus & India & Leaf & I & {$[20]$} \\
\hline Phyllanthus urinaria & Sri Lanka & ND & $\mathrm{I}, \mathrm{E}$ & {$[18]$} \\
\hline \multicolumn{5}{|l|}{ Phytolaccaceae } \\
\hline Petiveria alliacea & Colombia, Nicaragua & Branch, leaf, root, whole plant & $\mathrm{I}, \mathrm{E}$ & {$[27,34,35]$} \\
\hline \multicolumn{5}{|l|}{ Pinaceae } \\
\hline Pinus roxburghii ${ }^{*}$ & Pakistan & Oil, resin, wood & $\mathrm{E}$ & {$[17,53]$} \\
\hline \multicolumn{5}{|l|}{ Piperaceae } \\
\hline Peperomia elsana & Colombia & Whole plant & $\mathrm{E}$ & {$[35]$} \\
\hline Peperomia pellucida & Nicaragua, Sri Lanka & Whole plant & $\mathrm{I}, \mathrm{E}$ & {$[18,27]$} \\
\hline Piper amalago & Mexico, Nicaragua & Leaf, root & I & {$[27,59]$} \\
\hline Piper arboreum* & Colombia & Branch, leaf & $\mathrm{E}$ & {$[35]$} \\
\hline Piper auritum ${ }^{\#}$ & Colombia, Nicaragua & Branch, leaf, stem, whole plant & $\mathrm{I}, \mathrm{E}$ & {$[27,34,35,44]$} \\
\hline Piper betle & Sri Lanka & ND & $\mathrm{I}, \mathrm{E}$ & {$[18]$} \\
\hline Piper chuvya & Sri Lanka & ND & $\mathrm{E}$ & {$[18]$} \\
\hline Piper confusionis & Peru & Leaf & $\mathrm{E}$ & [57] \\
\hline Piper coruscans ${ }^{\#}$ & Colombia & Branch, leaf, stem & $\mathrm{I}, \mathrm{E}$ & {$[35]$} \\
\hline Piper hispidum ${ }^{\#}$ & Colombia & Branch, leaf, stem & $\mathrm{I}, \mathrm{E}$ & {$[35]$} \\
\hline Piper longivillosum ${ }^{\#}$ & Colombia & Whole plant & $\mathrm{E}$ & {$[35]$} \\
\hline Piper longum* & Bangladesh, Sri Lanka & Flower, fruit, Latex, root & $\mathrm{E}$ & {$[18,30]$} \\
\hline Piper marginatum ${ }^{\#}$ & Brazil, Colombia & Branch, leaf, root, stem & $\mathrm{I}, \mathrm{E}$ & {$[35,50]$} \\
\hline Piper multiplinervium ${ }^{\#}$ & Colombia & Branch, leaf, stem & $\mathrm{I}, \mathrm{E}$ & {$[35]$} \\
\hline
\end{tabular}


TABle 1: Continued.

\begin{tabular}{|c|c|c|c|c|}
\hline Plant name & Countries & Parts used & Use & Reference(s) \\
\hline Piper nigrum & $\begin{array}{l}\text { Bangladesh, India, Sri } \\
\text { Lanka }\end{array}$ & Floral bud, flower, fruit, root & $\mathrm{I}, \mathrm{E}$ & {$[18,20,28,32,52]$} \\
\hline Piper peltatum ${ }^{\#}$ & Colombia, Nicaragua & Branch, leaf, stem, whole plant & $\mathrm{I}, \mathrm{E}$ & {$[27,35]$} \\
\hline Piper pulchrum* & Colombia & Branch, leaf, stem & $\mathrm{I}, \mathrm{E}$ & {$[35]$} \\
\hline Piper reticulatum ${ }^{\#}$ & Colombia & Branch, leaf, stem & $\mathrm{I}, \mathrm{E}$ & [35] \\
\hline Piper tricuspe & Colombia & Branch, leaf, stem & $\mathrm{E}$ & [35] \\
\hline Piper umbellatum & Sri Lanka & ND & I, E & {$[18]$} \\
\hline \multicolumn{5}{|l|}{ Pittosporaceae } \\
\hline Pittosporum neelgherrense & India & Bark & $\mathrm{I}, \mathrm{E}$ & {$[16]$} \\
\hline Pittosporum tetraspermum & India & Bark & I & {$[26]$} \\
\hline \multicolumn{5}{|l|}{ Plantaginaceae } \\
\hline Bacopa monnieri & $\begin{array}{l}\text { Bangladesh, India, Sri } \\
\text { Lanka }\end{array}$ & Leaf, root, whole plant & $\mathrm{I}$ & {$[18,23,32,39,41]$} \\
\hline Plantago australis & Colombia & Whole plant & $\mathrm{E}$ & {$[34]$} \\
\hline Plantago major & Colombia & Aerial parts, leaf & $\mathrm{I}, \mathrm{E}$ & {$[44]$} \\
\hline Scoparia dulcis ${ }^{\#}$ & Colombia, Nicaragua & $\begin{array}{c}\text { Aerial parts, branch, leaf, root, } \\
\text { whole plant }\end{array}$ & $\mathrm{I}, \mathrm{E}$ & {$[27,34,35,44]$} \\
\hline \multicolumn{5}{|l|}{ Platanaceae } \\
\hline Platanus orientalis & Pakistan & Bark & $\mathrm{I}, \mathrm{E}$ & {$[17]$} \\
\hline \multicolumn{5}{|l|}{ Plumbaginaceae } \\
\hline Plumbago indica & Sri Lanka & ND & $\mathrm{I}, \mathrm{E}$ & {$[18]$} \\
\hline Plumbago zeylanica & $\begin{array}{l}\text { Bangladesh, India, Sri } \\
\text { Lanka }\end{array}$ & Root & $\mathrm{I}, \mathrm{E}$ & {$[18,23,32]$} \\
\hline \multicolumn{5}{|l|}{ Poaceae } \\
\hline $\begin{array}{l}\text { Chrysopogon zizanioides (syn. } \\
\text { Vetiveria zizanioides) }\end{array}$ & India, Sri Lanka & Root & $\mathrm{I}, \mathrm{E}$ & {$[16,18]$} \\
\hline Cymbopogon citratus & Colombia & Leaf & $\mathrm{E}$ & {$[34]$} \\
\hline Cynodon dactylon & $\begin{array}{l}\text { Bangladesh, India, Sri } \\
\text { Lanka }\end{array}$ & Leaf, root, whole plant & $\mathrm{E}$ & {$[18,19,32]$} \\
\hline Drynaria quercifolia & Sri Lanka & ND & I & {$[18]$} \\
\hline Eleusine coracana & Sri Lanka & $\mathrm{ND}$ & I & {$[18]$} \\
\hline Gynerium sagittatum & Nicaragua & Leaf, root & I & [27] \\
\hline Heteropogon contortus & India, Sri Lanka & Root & $\mathrm{I}, \mathrm{E}$ & {$[18,38,55]$} \\
\hline Isachne globosa & Sri Lanka & ND & $\mathrm{E}$ & [18] \\
\hline Oryza punctata & Sri Lanka & ND & I, E & {$[18]$} \\
\hline Oryza sativa & Sri Lanka & ND & I & {$[18]$} \\
\hline Pogonatherum paniceum & Sri Lanka & ND & $\mathrm{E}$ & {$[18]$} \\
\hline Saccharum arundinaceum & Sri Lanka & ND & $\mathrm{I}$ & [18] \\
\hline Saccharum officinarum & Colombia, Sri Lanka & Stem & I, E & {$[18,34,44]$} \\
\hline \multicolumn{5}{|l|}{ Polygalaceae } \\
\hline Polygala abyssinica & Pakistan & Root & I & [17] \\
\hline Polygala crotalarioides & India & Leaf, root & ND & [19] \\
\hline Polygala paniculata & Brazil & Root & $\mathrm{E}$ & [47] \\
\hline Polygala spectabilis & Brazil & Root & $\mathrm{I}, \mathrm{E}$ & [47] \\
\hline \multicolumn{5}{|l|}{ Polygonaceae } \\
\hline $\begin{array}{l}\text { Persicaria barbata (syn. Polygonum } \\
\text { barbatum) }\end{array}$ & India & Leaf & $\mathrm{I}, \mathrm{E}$ & {$[38]$} \\
\hline $\begin{array}{l}\text { Persicaria chinensis (syn. Polygonum } \\
\text { chinense) }\end{array}$ & Bangladesh & Leaf & $\mathrm{E}$ & {$[32]$} \\
\hline
\end{tabular}


TABLE 1: Continued.

\begin{tabular}{|c|c|c|c|c|}
\hline Plant name & Countries & Parts used & Use & Reference(s) \\
\hline $\begin{array}{l}\text { Persicaria ferruginea (syn. } \\
\text { Polygonum ferrugineum) }\end{array}$ & Colombia & Aerial parts & $\mathrm{E}$ & {$[34]$} \\
\hline $\begin{array}{l}\text { Persicaria glabra (syn. Polygonum } \\
\text { glabrum) }\end{array}$ & India & Root & $\mathrm{E}$ & {$[25]$} \\
\hline \multicolumn{5}{|l|}{ Polypodiaceae } \\
\hline Pleopeltis percussa* & Colombia & Branch, leaf, stem & $\mathrm{I}, \mathrm{E}$ & {$[35]$} \\
\hline Pyrrosia piloselloides & Sri Lanka & ND & $\mathrm{E}$ & {$[18]$} \\
\hline \multicolumn{5}{|l|}{ Pontederiaceae } \\
\hline Monochoria hastata & Sri Lanka & ND & $\mathrm{I}, \mathrm{E}$ & {$[18]$} \\
\hline \multicolumn{5}{|l|}{ Portulacaceae } \\
\hline Portulaca pilosa & Brazil & Leaf & I & {$[33]$} \\
\hline \multicolumn{5}{|l|}{ Primulaceae } \\
\hline Aegiceras corniculatum & Sri Lanka & ND & $\mathrm{E}$ & {$[18]$} \\
\hline Anagallis arvensis & Serbia & Aerial parts & ND & {$[58]$} \\
\hline Ardisia humilis & Sri Lanka & ND & $\mathrm{E}$ & {$[18]$} \\
\hline Maesa lanceolata* & Kenya & Root & ND & {$[31]$} \\
\hline Myrsine coriacea & Colombia & Whole plant & $\mathrm{E}$ & {$[34]$} \\
\hline \multicolumn{5}{|l|}{ Pteridaceae } \\
\hline Acrostichum aureum & Nicaragua & Leaf, root & $\mathrm{I}, \mathrm{E}$ & {$[27]$} \\
\hline Adiantum capillus-veneris & Pakistan & Frond & $\mathrm{E}$ & {$[17]$} \\
\hline Pellaea viridis & Kenya & Leaf & $\mathrm{E}$ & {$[31]$} \\
\hline \multicolumn{5}{|l|}{ Ranunculaceae } \\
\hline $\begin{array}{l}\text { Clematis brachiata (syn. Clematis } \\
\text { triloba) }\end{array}$ & India & Root & $\mathrm{E}$ & {$[25]$} \\
\hline Delphinium denudatum & India & Root & ND & {$[19]$} \\
\hline Delphinium vestitum & India & Whole plant & ND & {$[19]$} \\
\hline \multicolumn{5}{|l|}{ Rhamnaceae } \\
\hline Alphitonia incana & Papua New Guinea & Oil & $\mathrm{E}$ & {$[46]$} \\
\hline $\begin{array}{l}\text { Ziziphus jujuba (syn. Ziziphus } \\
\text { mauritiana) }\end{array}$ & Sri Lanka & ND & $\mathrm{E}$ & {$[18]$} \\
\hline Ziziphus oenoplia & India, Sri Lanka & Leaf & $\mathrm{I}, \mathrm{E}$ & {$[18,49]$} \\
\hline \multicolumn{5}{|l|}{ Rhizophoraceae } \\
\hline Rhizophora mangle & Nicaragua & Bark & $\mathrm{I}, \mathrm{E}$ & {$[27]$} \\
\hline \multicolumn{5}{|l|}{ Rosaceae } \\
\hline Crataegus monogyna & Spain & Thorn & ND & {$[37]$} \\
\hline Potentilla sundaica & India & Root, stem & ND & {$[19]$} \\
\hline Prunus persica & Ethiopia & Leaf & $\mathrm{I}$ & {$[51]$} \\
\hline Prunus walkeri & Sri Lanka & ND & $\mathrm{E}$ & {$[18]$} \\
\hline Pyrus communis & Pakistan & Fruit, leaf & $\mathrm{I}$ & {$[17]$} \\
\hline Sanguisorba officinalis & Serbia & Rhizome & ND & {$[58]$} \\
\hline \multicolumn{5}{|l|}{ Rubiaceae } \\
\hline $\begin{array}{l}\text { Catunaregam spinosa (syn. Randia } \\
\text { dumetorum) }\end{array}$ & India & Root & I & {$[23]$} \\
\hline $\begin{array}{l}\text { Ceriscoides turgida (syn. Gardenia } \\
\text { turgida) }\end{array}$ & India & Bark, root & $\mathrm{I}$ & {$[24,38]$} \\
\hline Chiococca alba & Brazil, Nicaragua & Leaf, root & $\mathrm{I}$ & {$[27,47]$} \\
\hline Clausena dentata & Sri Lanka & ND & $\mathrm{E}$ & [18] \\
\hline Gonzalagunia panamensis* & Colombia & Branch, leaf, stem & $\mathrm{I}, \mathrm{E}$ & [35] \\
\hline Hamelia axillaris & Nicaragua & Leaf, whole plant & $\mathrm{I}, \mathrm{E}$ & {$[27]$} \\
\hline Hamelia barbata & Nicaragua & Leaf, whole plant & $\mathrm{I}, \mathrm{E}$ & {$[27]$} \\
\hline Hamelia patens & Nicaragua & Leaf, whole plant & $\mathrm{I}, \mathrm{E}$ & {$[27]$} \\
\hline
\end{tabular}


TABle 1: Continued.

\begin{tabular}{|c|c|c|c|c|}
\hline Plant name & Countries & Parts used & Use & Reference(s) \\
\hline Hamelia rovirosae & Nicaragua & Flower, leaf, stem & $\mathrm{I}, \mathrm{E}$ & {$[27]$} \\
\hline Hedyotis scandens & Bangladesh & Leaf, stem & $\mathrm{E}$ & {$[32]$} \\
\hline Ixora coccinea & Sri Lanka & ND & $\mathrm{I}, \mathrm{E}$ & {$[18]$} \\
\hline Ixora cuneifolia & Bangladesh & Bark & $\mathrm{E}$ & {$[32]$} \\
\hline Ixora pavetta (syn. Ixora arborea) & India & Leaf, rood, seed & ND & [19] \\
\hline Mitragyna parvifolia & India & Bark, stem & $\mathrm{I}, \mathrm{E}$ & {$[38,63]$} \\
\hline Morinda angustifolia & Bangladesh & Leaf & I & [32] \\
\hline Morinda citrifolia & Bangladesh & Root & ND & {$[30]$} \\
\hline Morinda coreia & Sri Lanka & ND & $\mathrm{I}, \mathrm{E}$ & {$[18]$} \\
\hline Morinda persicifolia & Bangladesh & Leaf & $\mathrm{E}$ & {$[32]$} \\
\hline Mussaenda frondosa & Sri Lanka & ND & I & {$[18]$} \\
\hline Mussaenda roxburghii & Bangladesh & Leaf & $\mathrm{E}$ & {$[32]$} \\
\hline Nauclea orientalis & Sri Lanka & ND & $\mathrm{E}$ & {$[18]$} \\
\hline $\begin{array}{l}\text { Neonauclea purpurea (syn. } \\
\text { Anthocephalus chinensis) }\end{array}$ & Bangladesh & Bark, leaf & ND & {$[30]$} \\
\hline Oldenlandia diffusa & India & Whole plant & $\mathrm{E}$ & {$[20]$} \\
\hline Oldenlandia umbellata & India & Leaf, root & $\mathrm{E}$ & {$[20]$} \\
\hline Ophiorrhiza mungos* & India & Root & I & {$[16,20]$} \\
\hline Paederia foetida & Sri Lanka & ND & $\mathrm{I}, \mathrm{E}$ & {$[18]$} \\
\hline Palicourea croceoides & Colombia & Bark & I & {$[34]$} \\
\hline Pavetta indica & Sri Lanka & ND & $\mathrm{I}, \mathrm{E}$ & {$[18]$} \\
\hline Psychotria elata & Nicaragua & $\begin{array}{l}\text { Flower, leaf, root, stem, whole } \\
\text { plant }\end{array}$ & $\mathrm{I}, \mathrm{E}$ & {$[27]$} \\
\hline Psychotria flavida & India & Root & I & {$[63]$} \\
\hline Psychotria poeppigiana ${ }^{\#}$ & $\begin{array}{l}\text { Colombia, Nicaragua, } \\
\text { Sri Lanka }\end{array}$ & Branch, leaf, stem, whole plant & $\mathrm{I}, \mathrm{E}$ & {$[18,27,35]$} \\
\hline Randia aculeata ${ }^{*}$ & Mexico & Fruit, whole plant & I & {$[59,78]$} \\
\hline Rubia cordifolia* & Nepal, Pakistan & Leaf, root, stem & I & {$[17,69]$} \\
\hline Rubia manjith & India & Root, stem & ND & [19] \\
\hline $\begin{array}{l}\text { Spermacoce remota (syn. Borreria } \\
\text { assurgens) }\end{array}$ & Nicaragua & Leaf, root & $\mathrm{I}, \mathrm{E}$ & {$[27]$} \\
\hline Tamilnadia uliginosa & Sri Lanka & ND & I & {$[18]$} \\
\hline Wendlandia exserta & India & Root & I & {$[49]$} \\
\hline \multicolumn{5}{|l|}{ Rutaceae } \\
\hline Acronychia pedunculata & Sri Lanka & ND & $\mathrm{E}$ & {$[18]$} \\
\hline Aegle marmelos & $\begin{array}{l}\text { Bangladesh, India, Sri } \\
\text { Lanka }\end{array}$ & Bark, whole plant & $\mathrm{I}, \mathrm{E}$ & {$[18,20,30,32,41]$} \\
\hline Atalantia ceylanica & Sri Lanka & ND & $\mathrm{I}, \mathrm{E}$ & {$[18]$} \\
\hline Citrus aurantiifolia & Sri Lanka & ND & $\mathrm{I}, \mathrm{E}$ & {$[18]$} \\
\hline Citrus aurantium & Sri Lanka & $\mathrm{ND}$ & $\mathrm{I}, \mathrm{E}$ & {$[18]$} \\
\hline $\begin{array}{l}\text { Citrus japonica (syn. Citrus } \\
\text { madurensis) }\end{array}$ & Sri Lanka & ND & $\mathrm{I}, \mathrm{E}$ & {$[18]$} \\
\hline Citrus limon ${ }^{*}$ & $\begin{array}{l}\text { Colombia, India, Sri } \\
\text { Lanka }\end{array}$ & Fruit, leaf, root & $\mathrm{I}, \mathrm{E}$ & {$[18,20,28,34,35]$} \\
\hline Citrus maxima (syn. Citrus grandis) & Sri Lanka & ND & $\mathrm{I}, \mathrm{E}$ & {$[18]$} \\
\hline Glycosmis pentaphylla & India & Leaf & $\mathrm{I}, \mathrm{E}$ & {$[16]$} \\
\hline $\begin{array}{l}\text { Limonia acidissima (syn. Feronia } \\
\text { limonia) }\end{array}$ & India, Sri Lanka & Root & I & {$[18,20]$} \\
\hline Murraya koenigii & India, Sri Lanka & Bark, leaf & $\mathrm{I}, \mathrm{E}$ & {$[18,28]$} \\
\hline Murraya paniculata* & Sri Lanka & ND & $\mathrm{E}$ & {$[18]$} \\
\hline
\end{tabular}


TABLE 1: Continued.

\begin{tabular}{|c|c|c|c|c|}
\hline Plant name & Countries & Parts used & Use & Reference(s) \\
\hline Naringi crenulata & India & Fruit & ND & {$[19]$} \\
\hline Pamburus missionis & Sri Lanka & ND & $\mathrm{E}$ & {$[18]$} \\
\hline Ruta chalepensis & Colombia & Whole plant & $\mathrm{E}$ & {$[34]$} \\
\hline Toddalia asiatica & India, Sri Lanka & Root & $\mathrm{I}, \mathrm{E}$ & {$[18,63]$} \\
\hline \multicolumn{5}{|l|}{ Salicaceae } \\
\hline Casearia grandiflora* & ND & Bark, leaf & ND & {$[79]$} \\
\hline $\begin{array}{l}\text { Casearia nigrescens (syn. Casearia } \\
\text { elliptica) }\end{array}$ & India & Bark, leaf & ND & {$[19]$} \\
\hline Casearia sylvestris ${ }^{*}$ & Brazil & Leaf, whole plant & $\mathrm{ND}$ & {$[47,79]$} \\
\hline Casearia tomentosa & India & Bark, root & $\mathrm{I}, \mathrm{E}$ & {$[49,79]$} \\
\hline Flacourtia indica & Bangladesh & Leaf & $\mathrm{E}$ & [32] \\
\hline \multicolumn{5}{|l|}{ Santalaceae } \\
\hline Santalum album & Sri Lanka & ND & $\mathrm{E}$ & {$[18]$} \\
\hline \multicolumn{5}{|l|}{ Sapindaceae } \\
\hline Allophylus cobbe & Sri Lanka & ND & $\mathrm{I}, \mathrm{E}$ & {$[18]$} \\
\hline Cardiospermum halicacabum & India, Sri Lanka & Leaf & $\mathrm{I}, \mathrm{E}$ & {$[18,28]$} \\
\hline Dodonaea viscosa & India & Leaf & $\mathrm{E}$ & {$[28]$} \\
\hline Harpullia arborea & Sri Lanka & ND & $\mathrm{I}, \mathrm{E}$ & {$[18]$} \\
\hline Sapindus emarginatus & India & Bark & I & {$[20]$} \\
\hline Sapindus mukorossi & India, Pakistan & Fruit, leaf, root, seed & $\mathrm{E}$ & {$[17,25]$} \\
\hline \multicolumn{5}{|l|}{ Sapotaceae } \\
\hline $\begin{array}{l}\text { Madhuca longifolia (syn. Madhuca } \\
\text { indica) }\end{array}$ & India, Sri Lanka & Bark, fruit, leaf, nut, root, seed & I, E & {$[18,20,23,25,32,38]$} \\
\hline Manilkara zapota & Mexico & Root & ND & {$[59]$} \\
\hline Mimusops elengi & Sri Lanka & ND & I & {$[18]$} \\
\hline \multicolumn{5}{|l|}{ Scrophulariaceae } \\
\hline Verbascum thapsus & India & Leaf & ND & {$[66]$} \\
\hline \multicolumn{5}{|l|}{ Selaginellaceae } \\
\hline Selaginella articulata ${ }^{\#}$ & Colombia & Whole plant & $\mathrm{I}, \mathrm{E}$ & {$[35]$} \\
\hline \multicolumn{5}{|l|}{ Simaroubaceae } \\
\hline Ailanthus excelsa & India & Bark & I & {$[38]$} \\
\hline Quassia amara & Colombia, Nicaragua & Root, stem, whole plant & $\mathrm{I}, \mathrm{E}$ & {$[27,35]$} \\
\hline Quassia indica & Sri Lanka & ND & I & [18] \\
\hline Simaba cedron ${ }^{\#}$ & Colombia & Seed, whole plant & $\mathrm{I}, \mathrm{E}$ & {$[34,35,44]$} \\
\hline \multicolumn{5}{|l|}{ Siparunaceae } \\
\hline Siparuna gesnerioides & Colombia & Leaf, root & I & {$[34,44]$} \\
\hline Siparuna thecaphora* & Colombia & Branch, leaf, stem & $\mathrm{I}, \mathrm{E}$ & [35] \\
\hline \multicolumn{5}{|l|}{ Smilacaceae } \\
\hline Smilax regelii & Nicaragua & Root & I & {$[27]$} \\
\hline Smilax spinosa & Nicaragua & Root & I & {$[27]$} \\
\hline \multicolumn{5}{|l|}{ Solanaceae } \\
\hline Atropa acuminata & Pakistan & Leaf, root & $\mathrm{E}$ & {$[17]$} \\
\hline $\begin{array}{l}\text { Capsicum annuum (syn. Capsicum } \\
\text { frutescens) }\end{array}$ & $\begin{array}{l}\text { Bangladesh, Colombia, } \\
\text { India, Sri Lanka }\end{array}$ & Fruit, root & $\mathrm{I}, \mathrm{E}$ & {$[18,19,25,32,34,35]$} \\
\hline Datura metel & $\begin{array}{l}\text { Bangladesh, Colombia, } \\
\text { India, Sri Lanka }\end{array}$ & Bark, flower, fruit, leaf, root, seed & I, E & $\begin{array}{c}{[18,22,23,25,28,30} \\
34]\end{array}$ \\
\hline Datura stramonium ${ }^{\#}$ & India & Root & $\mathrm{I}, \mathrm{E}$ & {$[38]$} \\
\hline Lycopersicon esculentum & Colombia & Leaf, stem, whole plant & $\mathrm{E}$ & {$[34]$} \\
\hline
\end{tabular}


TABle 1: Continued.

\begin{tabular}{|c|c|c|c|c|}
\hline Plant name & Countries & Parts used & Use & Reference(s) \\
\hline Nicotiana tabacum & $\begin{array}{l}\text { Colombia, India, } \\
\text { Nicaragua }\end{array}$ & Leaf & $\mathrm{I}, \mathrm{E}$ & {$[20,27,44]$} \\
\hline Solanum allophyllum ${ }^{\#}$ & Colombia & Branch, leaf, stem & $\mathrm{I}, \mathrm{E}$ & {$[35]$} \\
\hline $\begin{array}{l}\text { Solanum americanum (syn. } \\
\text { Solanum nigrum) }\end{array}$ & $\begin{array}{l}\text { Colombia, India, Sri } \\
\text { Lanka }\end{array}$ & Fruit, leaf, whole plant & $\mathrm{I}, \mathrm{E}$ & {$[18,25,34,38]$} \\
\hline Solanum capsicoides & Bangladesh & Seed & ND & {$[30]$} \\
\hline Solanum incanum & Kenya & Fruit, stem & $\mathrm{E}$ & {$[31]$} \\
\hline Solanum melongena & Sri Lanka & ND & I & {$[18]$} \\
\hline Solanum nudum ${ }^{\#}$ & Colombia & Branch, fruit, leaf, stem & $\mathrm{I}, \mathrm{E}$ & {$[35]$} \\
\hline Solanum ochraceo-ferrugineum & Mexico & Whole plant & ND & {$[59]$} \\
\hline Solanum torvum & $\begin{array}{l}\text { Bangladesh, India, } \\
\text { Nicaragua }\end{array}$ & Flower, leaf, root & $\mathrm{I}, \mathrm{E}$ & {$[20,27,30,32]$} \\
\hline Solanum verbascifolium & Mexico & Whole plant & ND & [59] \\
\hline $\begin{array}{l}\text { Solanum virginianum (syn. Solanum } \\
\text { xanthocarpum) }\end{array}$ & India, Sri Lanka & Root & I & {$[18,41]$} \\
\hline Withania somnifera & $\begin{array}{l}\text { Bangladesh, India, Sri } \\
\text { Lanka }\end{array}$ & Root & $\mathrm{I}, \mathrm{E}$ & {$[18,22,32]$} \\
\hline \multicolumn{5}{|l|}{ Sterculiaceae } \\
\hline Byttneria pilosa & Bangladesh & Leaf, stem & $\mathrm{E}$ & {$[32]$} \\
\hline \multicolumn{5}{|l|}{ Symplocaceae } \\
\hline Symplocos cochinchinensis & Sri Lanka & ND & $\mathrm{E}$ & {$[18]$} \\
\hline Symplocos racemosa & Sri Lanka & ND & $\mathrm{I}, \mathrm{E}$ & {$[18]$} \\
\hline \multicolumn{5}{|l|}{ Talinaceae } \\
\hline Talinum paniculatum & Sri Lanka & ND & $\mathrm{E}$ & {$[18]$} \\
\hline \multicolumn{5}{|l|}{ Thymelaeaceae } \\
\hline Daphne papyracea & Pakistan & Leaf, root, stem & ND & {$[17]$} \\
\hline \multicolumn{5}{|l|}{ Triuridaceae } \\
\hline Sciaphila purpurea & Colombia & Whole plant & $\mathrm{I}, \mathrm{E}$ & {$[35]$} \\
\hline \multicolumn{5}{|l|}{ Urticaceae } \\
\hline Boehmeria nivea & Sri Lanka & ND & $\mathrm{E}$ & {$[18]$} \\
\hline Cecropia obtusifolia & Nicaragua & Leaf & I & [27] \\
\hline Cecropia peltata & Bangladesh, Nicaragua & Leaf, whole plant & $\mathrm{I}$ & {$[27,30]$} \\
\hline Girardinia diversifolia & Sri Lanka & ND & $\mathrm{E}$ & {$[18]$} \\
\hline $\begin{array}{l}\text { Pouzolzia zeylanica (syn. Pouzolzia } \\
\text { indica) }\end{array}$ & Bangladesh, India & Leaf, whole plant & $\mathrm{E}$ & {$[32,42]$} \\
\hline \multicolumn{5}{|l|}{ Verbenaceae } \\
\hline Aloysia triphylla & Colombia & Aerial parts & $\mathrm{E}$ & {$[34]$} \\
\hline Lantana camara & $\begin{array}{l}\text { Bangladesh, Colombia, } \\
\text { India, Sri Lanka }\end{array}$ & Flower, leaf, root, stem & $\mathrm{I}, \mathrm{E}$ & {$[18,32,34,39,41]$} \\
\hline Lippia alba & Colombia & Aerial parts, leaf, whole plant & $\mathrm{I}, \mathrm{E}$ & {$[34]$} \\
\hline Lippia grandis & Brazil & Leaf & I & {$[33]$} \\
\hline Stachytarpheta cayennensis & Colombia & Whole plant & $\mathrm{E}$ & {$[34]$} \\
\hline Verbena litoralis & Colombia & Fruit peel, leaf, root, seed & $\mathrm{E}$ & {$[34]$} \\
\hline Verbena officinalis & Pakistan & Root, whole plant & $\mathrm{E}$ & {$[17,53]$} \\
\hline \multicolumn{5}{|l|}{ Vitaceae } \\
\hline Ampelocissus latifolia & India & Root & I & {$[49]$} \\
\hline Cayratia pedata (syn. Cissus pedata) & Bangladesh & Leaf & ND & {$[30]$} \\
\hline Cayratia trifolia (syn. Vitis trifolia) & Bangladesh, India & Leaf, root & $\mathrm{I}, \mathrm{E}$ & {$[36,38]$} \\
\hline
\end{tabular}


TABLE 1: Continued.

\begin{tabular}{|c|c|c|c|c|}
\hline Plant name & Countries & Parts used & Use & Reference(s) \\
\hline Cissus adnata & Bangladesh & Leaf & $\mathrm{E}$ & {$[32]$} \\
\hline Cissus javana & Bangladesh & Leaf, stem & $\mathrm{E}$ & {$[32]$} \\
\hline Cissus quadrangularis ${ }^{\#}$ & Sri Lanka & ND & $\mathrm{E}$ & {$[18]$} \\
\hline Leea indica & Sri Lanka & ND & $\mathrm{E}$ & {$[18]$} \\
\hline Vitis heyneana (syn. Vitis lanata) & Bangladesh & Leaf & $\mathrm{E}$ & {$[32]$} \\
\hline \multicolumn{5}{|l|}{ Xanthorrhoeaceae } \\
\hline Aloe harlana & Ethiopia & Leaf & I & {$[51]$} \\
\hline Aloe littoralis & Pakistan & Whole plant & $\mathrm{E}$ & {$[17]$} \\
\hline Aloe vera & Nicaragua, Sri Lanka & Leaf & $\mathrm{I}, \mathrm{E}$ & {$[18,27]$} \\
\hline \multicolumn{5}{|l|}{ Zingiberaceae } \\
\hline Alpinia calcarata & Sri Lanka & ND & $\mathrm{I}, \mathrm{E}$ & {$[18]$} \\
\hline Alpinia galanga & Sri Lanka & ND & I & {$[18]$} \\
\hline Alpinia nigra & Sri Lanka & ND & $\mathrm{E}$ & {$[18]$} \\
\hline Alpinia purpurata & Colombia & Leaf & $\mathrm{E}$ & {$[44]$} \\
\hline Curcuma angustifolia & India & Rhizome & $\mathrm{E}$ & {$[28]$} \\
\hline Curcuma longa* & $\begin{array}{l}\text { Bangladesh, India, Sri } \\
\text { Lanka }\end{array}$ & Rhizome & $\mathrm{I}, \mathrm{E}$ & {$[16,18,20,30]$} \\
\hline Elettaria cardamomum & Sri Lanka & ND & $\mathrm{I}$ & {$[18]$} \\
\hline $\begin{array}{l}\text { Globba marantina (syn. Globba } \\
\text { bulbifera) }\end{array}$ & India & Rhizome & I & [49] \\
\hline Hedychium coronarium & Colombia, Nicaragua & Root, whole plant & $\mathrm{E}$ & {$[27,34]$} \\
\hline Renealmia alpinia* & Colombia & Rhizome, stem & $\mathrm{I}, \mathrm{E}$ & {$[34,35]$} \\
\hline Renealmia thyrsoidea & Colombia & Leaf, stem & I & [34] \\
\hline Zingiber officinale & $\begin{array}{c}\text { Ecuador, Nicaragua, Sri } \\
\text { Lanka }\end{array}$ & Rhizome, root & $\mathrm{I}, \mathrm{E}$ & {$[18,27,61]$} \\
\hline \multicolumn{5}{|l|}{ Zygophyllaceae } \\
\hline Balanites aegyptiaca ${ }^{\#}$ & India & Bark, fruit & $\mathrm{E}$ & {$[38]$} \\
\hline
\end{tabular}

In parentheses is the synonym used in the original work; out of the parentheses is the accepted name (in case of more than one paper treating the same species with different names); ND = information not described in the work; $\mathrm{I}=$ internal use; $\mathrm{E}=$ external use. * Species evaluated on antiophidic activities in previous studies (see Tables 2-8) showing good inhibitory potential against venom induced local effects. " Species evaluated on antiophidic activities in previous studies, however, with poor inhibition potential against venom induced local effects.

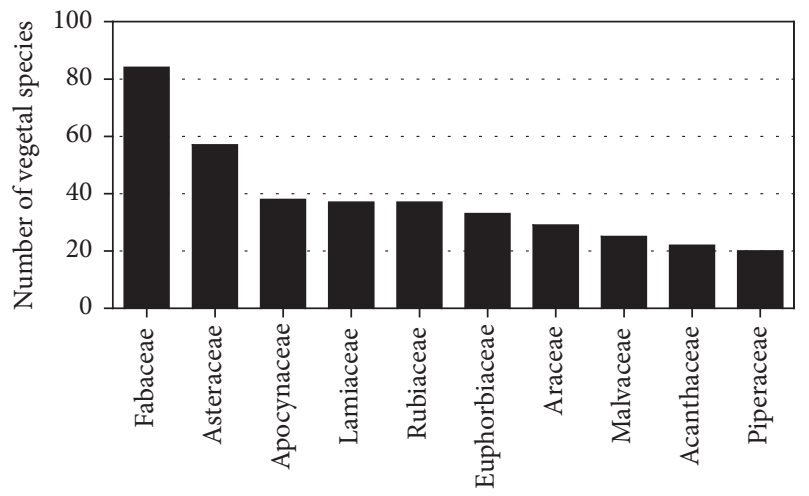

(a)

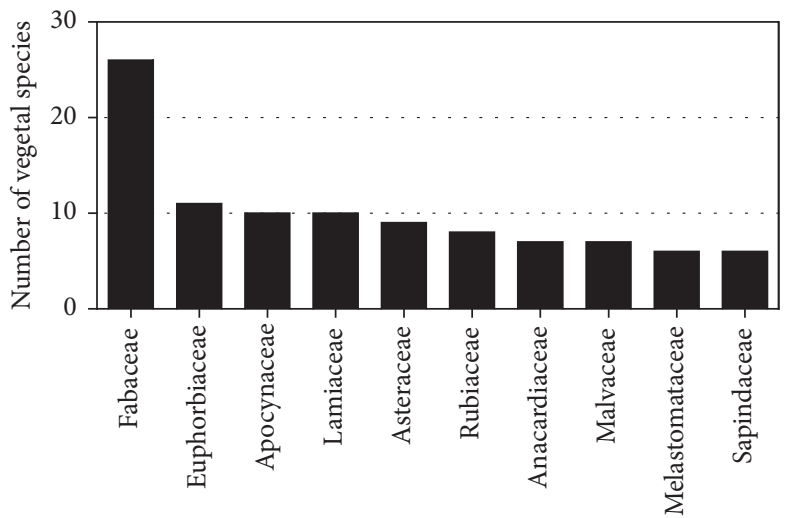

(b)

FIGURE 1: "Hot families" with antiophidic potential. Main related botanical families in ethnopharmacological surveys as antiophidic (a) and main botanical families that were evaluated in antiophidic assay (inhibition of local tissue damage) and presented positive results (b). 


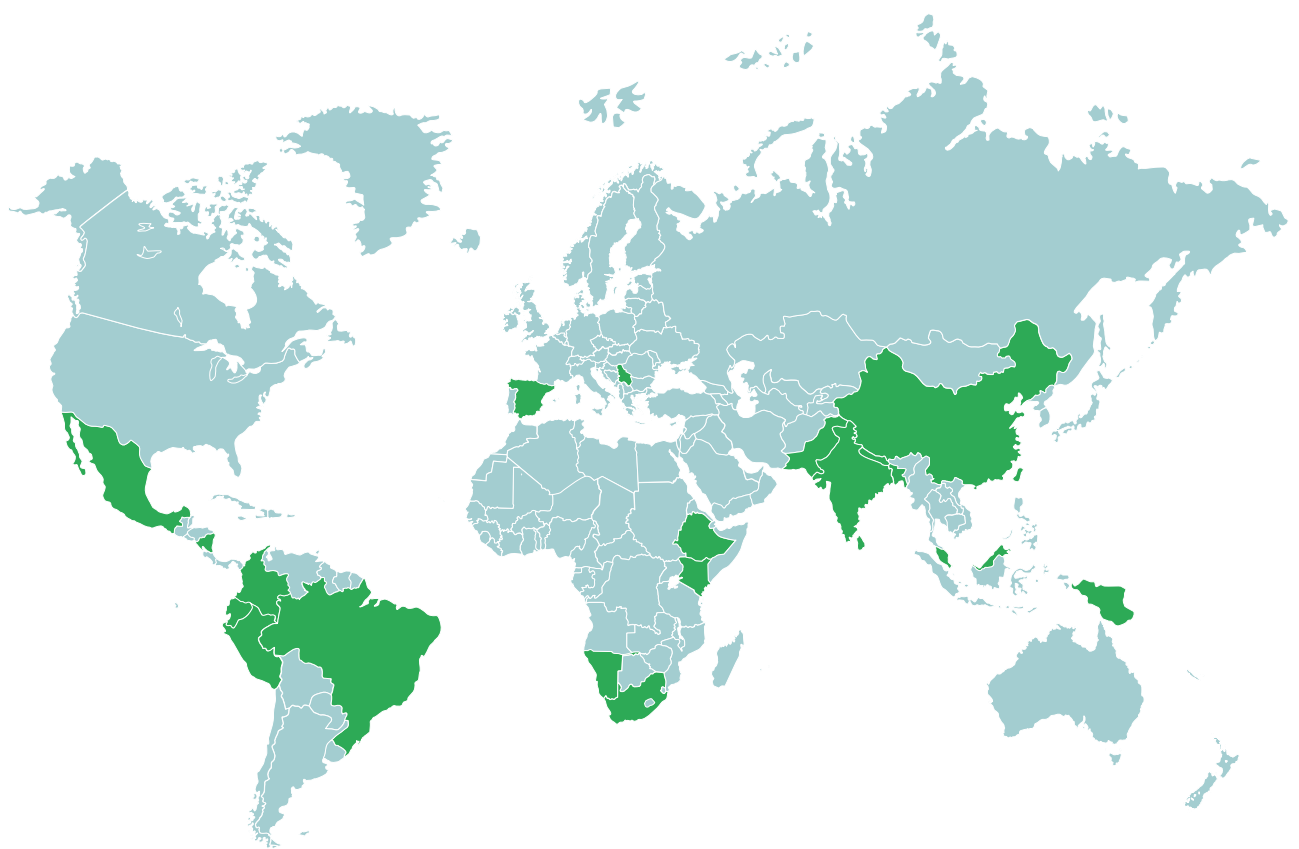

(a)

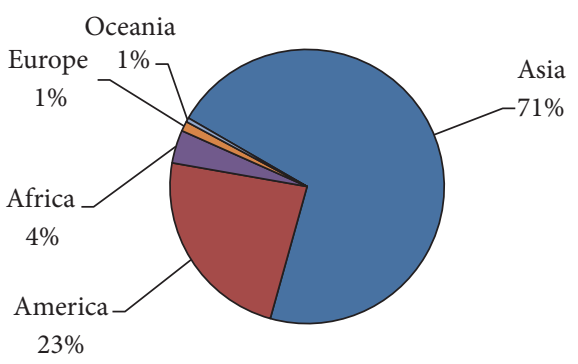

(b)

FIGURE 2: Distribution of medicinal plants used against snakebite around the world. World map highlighting the countries where antiophidic plants were related in ethnopharmacological surveys (a) and number of vegetal species per continent (b).

are very relevant, since they are involved in several pathological mechanisms produced by snake venoms; however, in vivo preclinical assays or, even better, clinical assays are essential for giving even stronger evidences of the effectivity of the use of medicinal plants against snakebites. In this scenario, the study of anti-Bothrops plants is more advanced, since quantitatively a higher number of in vivo scientific evidences are found in literature. Going the same way, studies with plants inhibiting local tissue damage of Daboia/Vipera, Lachesis, and Crotalus snakes could be also highlighted. However, studies of antiophidic medicinal plants in humans are very scarce: only one clinical study was found in literature, evaluating the inhibitory properties of a polyherbal formulation against local effects from Chinese cobra bite (see Section 4.9).

Hereafter, we describe the main plants with inhibitory potential against local tissue damage induced by snake venoms. It is important to emphasize that the focus of this review is plants against local tissue damage, mainly due to severity of these effects (which could cause permanent disabilities in victims) and the poor effectiveness of available antivenoms against them. So, studies with plants against systemic effects induced by snake were not considered; in addition some plants herein described possess inhibitory action upon systemic effects, although not stated here. For example, the vegetal species Jatropha gossypiifolia (Euphorbiaceae), a medicinal plant studied very much by our research group, had showed significative inhibitory action upon hemostatic disorders induced by $B$. jararaca snake venom [96]. So, the antiophidic potential of this species (as well as some others) lies beyond the capacity of inhibit local tissue damage provoked by $B$. jararaca venom, although not described in this review.

In addition, it is important to analyze critically some works dealing with antiophidic activity of plant extracts, since some of them have limitations that could reduce, at least partially, the potentiality of these species. The major limitation is that various studies, especially the early ones, make the evaluation of the plants using a preincubation approach, which consists in the previous inactivation of venom by preincubating it with different proportions of the tested extracts. Although scientifically valid and even recommended by WHO for assessing antiophidic antivenoms [97], 
TABLE 2: List of medicinal plants with inhibitory potential against local effects induced by Naja snakes.

\begin{tabular}{|c|c|c|c|c|c|}
\hline \multirow{2}{*}{ Plant name } & \multirow{2}{*}{ Part used } & \multirow{2}{*}{ Snake venom } & \multicolumn{2}{|c|}{ Inhibited activities } & \multirow{2}{*}{ Reference(s) } \\
\hline & & & In vitro & In vivo & \\
\hline \multicolumn{6}{|l|}{ Acanthaceae } \\
\hline Andrographis stenophylla & Leaf & N. naja & - & Hemorrhage & {$[83]$} \\
\hline \multicolumn{6}{|l|}{ Amaranthaceae } \\
\hline Pupalia lappacea & Herbal & N. nigricollis & SVH & - & {$[82]$} \\
\hline \multicolumn{6}{|l|}{ Amaryllidaceae } \\
\hline Allium cepa $a^{\#}$ & Bulb & N. n. karachiensis & $\mathrm{PLA}_{2}$ & - & {$[84]$} \\
\hline Allium sativum $^{\#}$ & Bulb & N. n. karachiensis & $\mathrm{PLA}_{2}$ & - & {$[84]$} \\
\hline \multicolumn{6}{|l|}{ Anacardiaceae } \\
\hline Lannea acida & Cortex & N. nigricollis & SVH & - & {$[82]$} \\
\hline $\begin{array}{l}\text { Pistacia chinensis } \\
\text { subsp.integerrima }\end{array}$ & Gall & N. n. karachiensis & $\mathrm{PLA}_{2}$ & - & {$[84]$} \\
\hline Sclerocarya birrea & Cortex & N. nigricollis & SVH & - & {$[82]$} \\
\hline Spondias mombin ${ }^{\#}$ & Cortex, radix & N. nigricollis & SVH & - & {$[82]$} \\
\hline \multicolumn{6}{|l|}{ Annonaceae } \\
\hline Annona senegalensis ${ }^{\#}$ & Cortex & N. nigricollis & SVH & - & {$[82]$} \\
\hline \multicolumn{6}{|l|}{ Apiaceae } \\
\hline Cuminum cyminum & Seed & N.n. karachiensis & $\mathrm{PLA}_{2}$ & - & {$[84]$} \\
\hline \multicolumn{6}{|l|}{ Apocynaceae } \\
\hline Acokanthera oppositifolia & Radix & N. nigricollis & SVH & - & \\
\hline Calotropis procera ${ }^{\#}$ & Flower, latex & N.n. karachiensis & $\mathrm{PLA}_{2}$ & - & {$[84]$} \\
\hline Strophanthus sarmentosus & Folium & N. nigricollis & SVH & - & {$[82]$} \\
\hline Strophanthus speciosus & Radix & N. nigricollis & SVH & - & {$[82]$} \\
\hline Tylophora indica & Leaf, root & N. naja & $\mathrm{PLA}_{2}$ & Hemorrhage & {$[85]$} \\
\hline \multicolumn{6}{|l|}{ Araceae } \\
\hline Colocasia esculenta & Tuber & N. nigricollis & SVH & - & {$[82]$} \\
\hline \multicolumn{6}{|l|}{ Araliaceae } \\
\hline Polyscias fulva & Cortex & N. nigricollis & SVH & - & {$[82]$} \\
\hline \multicolumn{6}{|l|}{ Aristolochiaceae } \\
\hline Aristolochia bracteolata ${ }^{\#}$ & Leaf, root & N. naja & $\mathrm{PLA}_{2}$ & Hemorrhage & {$[85]$} \\
\hline \multicolumn{6}{|l|}{ Asteraceae } \\
\hline Callilepis laureola & Radix & N. nigricollis & SVH & - & {$[82]$} \\
\hline \multicolumn{6}{|l|}{ Bignoniaceae } \\
\hline Kigelia africana & Cortex, folium & N. nigricollis & SVH & - & {$[82]$} \\
\hline $\begin{array}{l}\text { Tecoma stans (syn. Stenolobium } \\
\text { stans) }\end{array}$ & Root & N.n. karachiensis & $\mathrm{PLA}_{2}$ & - & {$[84]$} \\
\hline \multicolumn{6}{|l|}{ Bixaceae } \\
\hline Cochlospermum tinctorium & Radix & N. nigricollis & SVH & - & {$[82]$} \\
\hline \multicolumn{6}{|l|}{ Boraginaceae } \\
\hline Cordia macleodii & Bark & N. naja & - & $\begin{array}{l}\text { Edema, hemorrhage* } \\
\text { necrosis }\end{array}$ & {$[86]$} \\
\hline Trichodesma indicum ${ }^{\#}$ & Whole plant & N.n. karachiensis & $\mathrm{PLA}_{2}$ & - & {$[84]$} \\
\hline \multicolumn{6}{|l|}{ Capparaceae } \\
\hline Capparis tomentosa & Radix & N. nigricollis & SVH & - & {$[82]$} \\
\hline \multicolumn{6}{|l|}{ Colchicaceae } \\
\hline Gloriosa superba & Radix & N. nigricollis & SVH & - & {$[82]$} \\
\hline \multicolumn{6}{|l|}{ Combretaceae } \\
\hline Combretum molle & Folium & N. nigricollis & SVH & - & {$[82]$} \\
\hline Guiera senegalensis & Radix & N. nigricollis & SVH & - & {$[82]$} \\
\hline Terminalia arjuna & Bark & N. n. karachiensis & $\mathrm{PLA}_{2}$ & - & {$[84]$} \\
\hline
\end{tabular}


TABle 2: Continued.

\begin{tabular}{|c|c|c|c|c|c|}
\hline \multirow{2}{*}{ Plant name } & \multirow{2}{*}{ Part used } & \multirow{2}{*}{ Snake venom } & \multicolumn{2}{|c|}{ Inhibited activities } & \multirow{2}{*}{ Reference(s) } \\
\hline & & & In vitro & In vivo & \\
\hline \multicolumn{6}{|l|}{ Convolvulaceae } \\
\hline Ipomoea rubens & Seed & N. nigricollis & SVH & - & {$[82]$} \\
\hline \multicolumn{6}{|l|}{ Cucurbitaceae } \\
\hline Citrullus colocynthis ${ }^{\#}$ & Fruit & N. n. karachiensis & $\mathrm{PLA}_{2}$ & - & {$[84]$} \\
\hline $\begin{array}{l}\text { Luffa cylindrica (syn. Luffa } \\
\text { aegyptiaca) }\end{array}$ & Leaf & N. nigricollis & Proteolytic & - & {$[87]$} \\
\hline Momordica charantia & Fruit & N. n. karachiensis & $\mathrm{PLA}_{2}$ & - & {$[84]$} \\
\hline \multicolumn{6}{|l|}{ Ebenaceae } \\
\hline Diospyros mespiliformis & Cortex & N. nigricollis & SVH & - & {$[82]$} \\
\hline \multicolumn{6}{|l|}{ Euphorbiaceae } \\
\hline Alchornea laxiflora & Cortex & N. nigricollis & SVH & - & {$[82]$} \\
\hline Clutia cordata & Radix & N. nigricollis & SVH & - & {$[82]$} \\
\hline Euphorbia hirta & Whole plant & N. naja & $\begin{array}{l}\mathrm{PLA}_{2} \text {, proteolytic, } \\
\text { SVH }\end{array}$ & Edema* & {$[88]$} \\
\hline Jatropha curcas $\#$ & Leaf, root, stem & N. naja & $\mathrm{PLA}_{2}$ & - & [89] \\
\hline Jatropha gossypiifolia & Leaf, root, stem & N. naja & $\mathrm{PLA}_{2}$ & - & [89] \\
\hline $\begin{array}{l}\text { Manihot foetida (syn. Jatropha } \\
\text { foetida) }\end{array}$ & Leaf, stem & N. naja & $\mathrm{PLA}_{2}$ & - & {$[89]$} \\
\hline \multicolumn{6}{|l|}{ Fabaceae } \\
\hline Abrus precatorius ${ }^{\#}$ & Radix & N. nigricollis & SVH & - & {$[82]$} \\
\hline Argyrolobium stipulaceum & Radix & N. nigricollis & SVH & - & {$[82]$} \\
\hline Bauhinia thonningii & Cortex, radix & N. nigricollis & SVH & - & {$[82]$} \\
\hline Bauhinia variegata ${ }^{\#}$ & Root & N.n. karachiensis & $\mathrm{PLA}_{2}$ & - & {$[84]$} \\
\hline $\begin{array}{l}\text { Bobgunnia madagascariensis (syn. } \\
\text { Swartzia madagascariensis) }\end{array}$ & Folium, radix & N. nigricollis & SVH & - & {$[82]$} \\
\hline Burkea africana & Cortex & N. nigricollis & SVH & - & {$[82]$} \\
\hline $\begin{array}{l}\text { Cullen corylifolium (syn. Psoralea } \\
\text { corylifolia) }\end{array}$ & Seed & N. n. karachiensis & $\mathrm{PLA}_{2}$ & - & {$[84]$} \\
\hline Dichrostachys cinerea & Folium & N. nigricollis & SVH & - & {$[82]$} \\
\hline Entada africana & Radix & N. nigricollis & SVH & - & {$[82]$} \\
\hline Mimosa pudica & Root & N. kaouthia, N. naja & $\begin{array}{l}\mathrm{PLA}_{2} \text {, proteolytic, } \\
\text { SVH }\end{array}$ & Edema* $^{*}$ myotoxicity ${ }^{*}$ & [90-92] \\
\hline Parkia biglobosa & $\begin{array}{l}\text { Cortex, stem } \\
\text { bark }\end{array}$ & N. nigricollis & $\begin{array}{l}\text { Cytotoxicity } \\
\text { against muscle } \\
\text { cells, SVH }\end{array}$ & & {$[82,93]$} \\
\hline Stylosanthes erecta & Folium & N. nigricollis & SVH & - & {$[82]$} \\
\hline Tamarindus indica & Folium, radix & N. nigricollis & SVH & - & {$[82]$} \\
\hline \multicolumn{6}{|l|}{ Gentianaceae } \\
\hline $\begin{array}{l}\text { Enicostema axillare (syn. } \\
\text { Enicostema hyssopifolium) }\end{array}$ & Whole plant & N.n. karachiensis & $\mathrm{PLA}_{2}$ & - & {$[84]$} \\
\hline \multicolumn{6}{|l|}{ Hypericaceae } \\
\hline \multicolumn{5}{|l|}{ Lamiaceae } & {$[82]$} \\
\hline Leucas aspera ${ }^{\#}$ & $\begin{array}{l}\text { Leaf, root, } \\
\text { whole plant }\end{array}$ & N. naja & $\begin{array}{l}\mathrm{PLA}_{2} \text {, proteolytic, } \\
\text { SVH }\end{array}$ & Hemorrhage & {$[85,94]$} \\
\hline $\begin{array}{l}\text { Leucas cephalotes (syn. Leucas } \\
\text { capitata) }\end{array}$ & Whole plant & N. n. karachiensis & $\mathrm{PLA}_{2}$ & - & {$[84]$} \\
\hline Leucas martinicensis & ND & N. nigricollis & SVH & - & {$[82]$} \\
\hline $\begin{array}{l}\text { Ocimum tenuiflorum (syn. Ocimum } \\
\text { sanctum) }\end{array}$ & Whole plant & N. n. karachiensis & $\mathrm{PLA}_{2}$ & - & {$[84]$} \\
\hline $\begin{array}{l}\text { Rotheca myricoides (syn. } \\
\text { Clerodendrum myricoides) }\end{array}$ & Cortex & N. nigricollis & SVH & - & {$[82]$} \\
\hline
\end{tabular}


TABLE 2: Continued.

\begin{tabular}{|c|c|c|c|c|c|}
\hline \multirow{2}{*}{ Plant name } & \multirow{2}{*}{ Part used } & \multirow{2}{*}{ Snake venom } & \multicolumn{2}{|c|}{ Inhibited activities } & \multirow{2}{*}{ Reference(s) } \\
\hline & & & In vitro & In vivo & \\
\hline Teucrium kraussii & $\begin{array}{l}\text { Aerial parts, } \\
\text { cortex }\end{array}$ & N. nigricollis & SVH & - & {$[82]$} \\
\hline $\begin{array}{l}\text { Volkameria glabra (syn. } \\
\text { Clerodendrum glabrum) }\end{array}$ & Radix & N. nigricollis & SVH & - & {$[82]$} \\
\hline \multicolumn{6}{|l|}{ Lauraceae } \\
\hline Cassytha filiformis & Herbal & N. nigricollis & SVH & - & {$[82]$} \\
\hline \multicolumn{6}{|l|}{ Loganiaceae } \\
\hline Strychnos innocua & Folium & N. nigricollis & SVH & - & {$[82]$} \\
\hline Strychnos nux-vomica ${ }^{\#}$ & Seed & N. kaouthia & $\mathrm{PLA}_{2}$ & - & {$[95]$} \\
\hline \multicolumn{6}{|l|}{ Malvaceae } \\
\hline Althaea officinalis & Root & N. n. karachiensis & $\mathrm{PLA}_{2}$ & - & {$[84]$} \\
\hline Dombeya quinqueseta & Cortex & N. nigricollis & SVH & - & {$[82]$} \\
\hline Grewia mollis & $\begin{array}{l}\text { Cortex, folium, } \\
\text { radix }\end{array}$ & N. nigricollis & SVH & - & {$[82]$} \\
\hline Sterculia setigera & Cortex & N. nigricollis & SVH & - & {$[82]$} \\
\hline Waltheria indica & Radix & N. nigricollis & SVH & - & {$[82]$} \\
\hline \multicolumn{6}{|l|}{ Menispermaceae } \\
\hline Cissampelos mucronata & Herbal & N. nigricollis & SVH & - & {$[82]$} \\
\hline \multicolumn{6}{|l|}{ Moraceae } \\
\hline Ficus platyphylla & Folium & N. nigricollis & SVH & - & {$[82]$} \\
\hline \multicolumn{6}{|l|}{ Olacaceae } \\
\hline Ximenia americana & Folium & N. nigricollis & SVH & - & {$[82]$} \\
\hline \multicolumn{6}{|l|}{ Pedaliaceae } \\
\hline Ceratotheca sesamoides & Herbal & N. nigricollis & SVH & - & {$[82]$} \\
\hline \multicolumn{6}{|l|}{ Peraceae } \\
\hline Clutia pulchella & Radix & N. nigricollis & SVH & - & {$[82]$} \\
\hline \multicolumn{6}{|l|}{ Phyllanthaceae } \\
\hline $\begin{array}{l}\text { Flueggea virosa (syn. Securinega } \\
\text { virosa) }\end{array}$ & Radix & N. nigricollis & SVH & - & {$[82]$} \\
\hline \multicolumn{6}{|l|}{ Pinaceae } \\
\hline Cedrus deodara & Bark & N. n. karachiensis & $\mathrm{PLA}_{2}$ & - & {$[84]$} \\
\hline Pinus roxburghii & Oleoresin & N. n. karachiensis & $\mathrm{PLA}_{2}$ & - & {$[84]$} \\
\hline \multicolumn{6}{|l|}{ Poaceae } \\
\hline Cymbopogon schoenanthus & Radix & N. nigricollis & SVH & - & {$[82]$} \\
\hline \multicolumn{6}{|l|}{ Primulaceae } \\
\hline Maesa lanceolata & Cortex & N. nigricollis & SVH & - & {$[82]$} \\
\hline \multicolumn{6}{|l|}{ Rhamnaceae } \\
\hline Ziziphus mucronata & Radix & N. nigricollis & SVH & - & {$[82]$} \\
\hline Ziziphus spina-christi & Cortex & N. nigricollis & SVH & - & {$[82]$} \\
\hline \multicolumn{6}{|l|}{ Rubiaceae } \\
\hline Crossopteryx febrifuga & Cortex & N. nigricollis & SVH & - & {$[82]$} \\
\hline Pentanisia prunelloides & Radix & N. nigricollis & SVH & - & {$[82]$} \\
\hline Pentas zanzibarica & Folium & N. nigricollis & SVH & - & {$[82]$} \\
\hline Rubia cordifolia & Stem & & & & \\
\hline \multicolumn{6}{|l|}{ Rutaceae } \\
\hline Citrus limon ${ }^{\#}$ & Fruit & N. n. karachiensis & $\mathrm{PLA}_{2}$ & - & {$[84]$} \\
\hline Zanthoxylum capense & Radix & N. nigricollis & SVH & - & {$[82]$} \\
\hline \multicolumn{6}{|l|}{ Sapindaceae } \\
\hline Paullinia pinnata & Folium & N. nigricollis & SVH & - & {$[82]$} \\
\hline Sapindus mukorossi & Fruit & N. n. karachiensis & $\mathrm{PLA}_{2}$ & - & {$[84]$} \\
\hline
\end{tabular}


TABLE 2: Continued.

\begin{tabular}{|c|c|c|c|c|c|}
\hline \multirow{2}{*}{ Plant name } & \multirow{2}{*}{ Part used } & \multirow{2}{*}{ Snake venom } & \multicolumn{2}{|c|}{ Inhibited activities } & \multirow{2}{*}{ Reference(s) } \\
\hline & & & In vitro & In vivo & \\
\hline \multicolumn{6}{|l|}{ Solanaceae } \\
\hline Nicotiana rustica & Leaf & N. nigricollis & Proteolytic & - & {$[87]$} \\
\hline Schwenckia americana & Folium & N. nigricollis & SVH & - & {$[82]$} \\
\hline \multicolumn{6}{|l|}{ Thymelaeaceae } \\
\hline Gnidia anthylloides & Radix & N. nigricollis & SVH & - & {$[82]$} \\
\hline Gnidia kraussiana & Radix & N. nigricollis & SVH & - & {$[82]$} \\
\hline Gnidia splendens & Radix & N. nigricollis & $\mathrm{SVH}$ & - & {$[82]$} \\
\hline \multicolumn{6}{|l|}{ Verbenaceae } \\
\hline Lantana trifolia & Cortex & N. nigricollis & SVH & - & {$[82]$} \\
\hline \multicolumn{6}{|l|}{ Vitaceae } \\
\hline Cissus populnea & Stem & N. nigricollis & SVH & - & {$[82]$} \\
\hline \multicolumn{6}{|l|}{ Zingiberaceae } \\
\hline Zingiber officinale & Rhizome & N. n. karachiensis & $\mathrm{PLA}_{2}$ & - & {$[84]$} \\
\hline \multicolumn{6}{|l|}{ Zygophyllaceae } \\
\hline Fagonia cretica & Leaf, stem & N. n. karachiensis & $\mathrm{PLA}_{2}$ & - & {$[84]$} \\
\hline
\end{tabular}

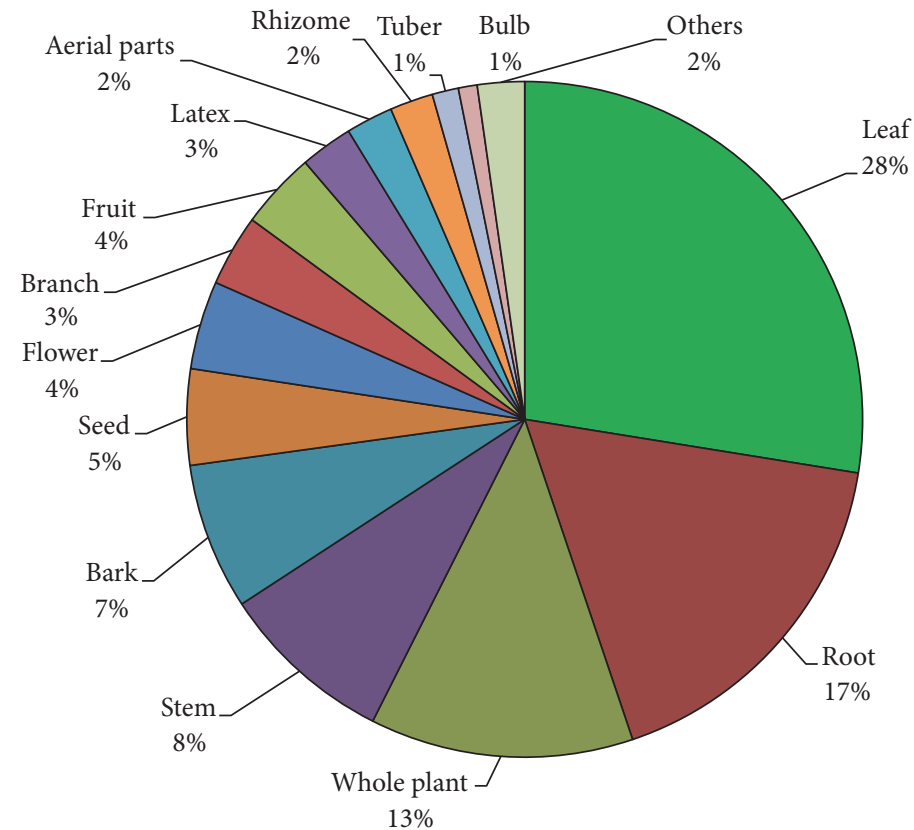

(a)

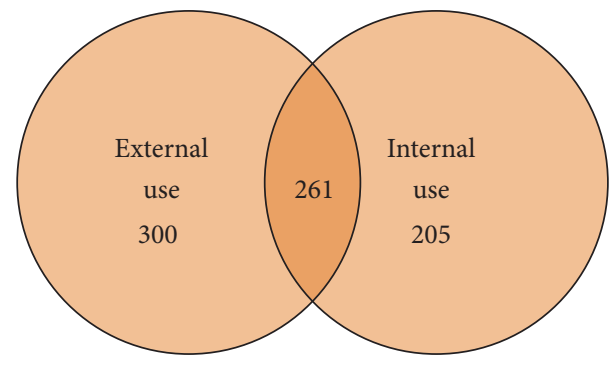

(b)

FIGURE 3: Mode of utilization of antiophidic plants reported by folk medicine. Main plant parts used (a) and Venn diagram showing the number of species enlisted having external use, internal use, or both (b). 
this preincubation approach makes a scenario unlikely to be possible in the field, where the medicine would be delivered after the snakebite. In fact, a recent study evaluated the inhibitory action of the medicinal plant Bellucia dichotoma (Melastomataceae) against Bothrops atrox snake venom using different protocols: preincubation, pretreatment, and posttreatment [98]. The authors observed that while the extract was greatly active when preincubated, this inhibitory activity was drastically reduced or even lost when the extract was injected independently of venom, simulating traditional use. The authors observed that the extract has great amounts of tannins, which are compounds known to precipitate proteins. So, it was concluded that the "pseudo-inhibition" observed after preincubation may be due to the presence of these compounds, suggesting that the preincubation protocol overestimates inhibitory potential of medicinal plants, and for this reason, this kind of approach must be analyzed with caution for estimation of inhibitory potential of medicinal plants $[13,98]$. In this sense, many recent studies have been done using protocols of pre- and/or posttreatment, to ensure the potentiality of antiophidic plants, and for most of them, positive results have been found [96, 98-102]. For this reason, studies using preincubation protocol are marked in the tables, for a critical analysis.

Also, it is interesting to note that several of the plants with inhibitory potential against snake venom local toxicities also present other relevant pharmacological activities. This is interesting since it is often discussed in the literature that several antiophidic plants did not neutralize snake venoms per se, but could have antiophidic use once they could relieve some of the symptoms of snake envenoming, especially the local effects. It is related that the presence of tranquilizing, antioxidant, immunostimulating, and/or antiinflammatory activities in certain plants could be of great interest in the alleviation of snake envenoming symptoms $[103,104]$. For example, some studies have shown that antiinflammatory drugs could inhibit the edematogenic and other snake venom effects related to inflammation, such as necrosis and myotoxicity, induced by Bothrops venoms [105, 106]. In fact, many medicinal plants with antiophidic activity also possess significant anti-inflammatory activity in vivo $[83,96,107-110]$. Following the same reasoning, some plants with antioxidant activity also possess significant antiophidic effects [95, 96, 104, 111]. In fact, some authors suggest that molecules with antioxidant and/or anti-inflammatory effects could be interesting along with antivenom therapy, helping to reduce the occurrence of secondary/long term complication due to snakebites [112].

Bacterial infection secondary to snakebites is a common complication in envenomed victims $[113,114]$. The main source of bacteria is the oral cavity of snakes, but the microbiota in the different layers of the victim's skin or even microorganisms from victim's clothes could also contribute $[115,116]$. Abscess formation is a common complication found in patients bitten by Viperidae snakes, being a risk factor for amputation in these patients, and it may be associated with sepsis $[113,114,117]$. A large number of bacteria, including anaerobic species, aerobic gram-negative rods, and a small proportion of gram-positive cocci could be inoculated with snakebites and have been isolated from the abscesses of bitten patients $[113,114]$. Microorganisms such as Staphylococcus, Pseudomonas, Salmonella, Escherichia, Providencia, Proteus, Enterococcus, and Bacillus were already identified in oral cavity of certain snakes [116]. The use of antibiotics following snakebites is often recommended, usually therapeutically than prophylactically, mainly to avoid complications due to infections $[114,118]$. In this context, medicinal plants presenting antimicrobial activities, especially against those microorganisms usually detected in snakebite victims' abscesses, could be interesting [115].

Medicinal plants having antimicrobial activities in association with some of the pharmacological properties discussed above (such as anti-inflammatory and antioxidant, e.g.) could be of great value to relieve especially local effects induced by snake venom. In another point of view, it is possible that several related plants in folk medicine as antiophidic agents do not act directly upon venom toxins but indirectly on its symptoms. Anyway, some studies have shown the potentiality of some vegetal species acting in two ways: directly, neutralizing venom toxins, or indirectly, by having some of the pharmacological activities mentioned above. For example, Jatropha gossypiifolia (Euphorbiaceae), a plant species studied very much in our research group, showed significant antiophidic properties, inhibiting biological and enzymatic activities from Bothrops venoms [96, 119], and presented anti-inflammatory, antioxidant, anticoagulant, and antimicrobial properties in preclinical assays [81]. So, plants which possess these biological activities determined in previous studies might be preferred or prioritized in studies searching for antiophidic plants.

The mechanism by which medicinal plants neutralize the toxic venom constituents is still unknown, but many hypotheses have been proposed, such as protein precipitation, enzyme inactivation, proteolytic degradation, metal chelation, antioxidant action, and a combination of these mechanisms [15]. In this context, some improvements in this understanding have been achieved in the last years, through the use of in silico methods (e.g., docking simulations) to analyze the interaction of compounds isolated from plants and certain classes of snake venom toxins such as $\mathrm{PLA}_{2}$ and SVMP [120-122].

The use of medicinal plants may present several advantages, such as low cost, being easily available, being stable at room temperature, and possibility of neutralization of a wide range of venom components [15]. In addition, since medicinal plants are an extremely complex mixture, it is possible that there may be a synergistic action of different compounds in plant, acting in distinct targets, inhibiting a broad spectrum of venom toxins $[12,15]$. According to literature, interestingly, there are some plants in which the crude extract is more active than the isolated constituents [15], which supports the hypothesis of the synergistic action of plant components.

4.2. Plants Inhibiting Naja Snakes. A summary of active plants against Naja snakes local effects is presented in Table 2. Naja species are commonly called cobras. They typically occur in regions throughout Africa and Southern Asia. The outcomes of venom toxicity include nephro-, neuro-, and 
TABLE 3: List of medicinal plants with inhibitory potential against local effects induced by Bothrops snakes.

\begin{tabular}{|c|c|c|c|c|c|}
\hline \multirow{2}{*}{ Plant name } & \multirow{2}{*}{ Part used } & \multirow{2}{*}{ Snake venom } & \multicolumn{2}{|c|}{ Inhibited activities } & \multirow{2}{*}{ Reference(s) } \\
\hline & & & In vitro & In vivo & \\
\hline \multicolumn{6}{|l|}{ Acanthaceae } \\
\hline Justicia pectoralis ${ }^{\#}$ & Leaf & B. jararaca & - & Hemorrhage $^{*}$ & {$[33]$} \\
\hline \multicolumn{6}{|l|}{ Amaranthaceae } \\
\hline Blutaparon portulacoides & Aerial parts & B. jararacussu & - & Edema & [109] \\
\hline \multicolumn{6}{|l|}{ Anacardiaceae } \\
\hline Anacardium excelsum & Leaf, twig & B. asper & $\mathrm{PLA}_{2}$ & - & {$[111]$} \\
\hline \multicolumn{6}{|l|}{ Annonaceae } \\
\hline Ephedranthus columbianus & Leaf, twig & B. asper & $\mathrm{PLA}_{2}$ & - & {$[111]$} \\
\hline Sapranthus isae & Leaf, twig & B. asper & $\mathrm{PLA}_{2}$ & - & {$[111]$} \\
\hline \multicolumn{6}{|l|}{ Apocynaceae } \\
\hline Allamanda cathartica ${ }^{\#}$ & $\begin{array}{l}\text { Branch, leaf, } \\
\text { stem }\end{array}$ & B. atrox & - & Hemorrhage* $^{*}$ & {$[124]$} \\
\hline $\begin{array}{l}\text { Fernaldia pandurata (syn. } \\
\text { Mandevilla velutina) }\end{array}$ & $\begin{array}{l}\text { Leaf, stem, } \\
\text { subterranean } \\
\text { system }\end{array}$ & $\begin{array}{l}\text { B. alternatus, } B . \\
\text { jararacussu, } B . \\
\text { moojeni, } B . \\
\text { pirajai }\end{array}$ & $\mathrm{PLA}_{2}$ & $\begin{array}{c}\text { Edema*, } \\
\text { hemorrhage*, } \\
\text { myotoxicity* }\end{array}$ & {$[125]$} \\
\hline Tabernaemontana catharinensis & Root bark & B. jararacussu & Myotoxicity & Myotoxicity $^{* *}$ & {$[126]$} \\
\hline \multicolumn{6}{|l|}{ Araceae } \\
\hline Dracontium croatii ${ }^{\#}$ & Rhizome & B. asper & - & Edema* & {$[127]$} \\
\hline Philodendron megalophyllum ${ }^{\#}$ & Liana, vine & $\begin{array}{l}\text { B. atrox, } B . \\
\text { jararaca }\end{array}$ & $\mathrm{PLA}_{2}$ & $\begin{array}{c}\text { Edema*, } \\
\text { hemorrhage* }\end{array}$ & {$[33,128]$} \\
\hline Philodendron tripartitum ${ }^{\#}$ & Branch, leaf & B. atrox & - & Hemorrhage $^{*}$ & {$[124]$} \\
\hline \multicolumn{6}{|l|}{ Asteraceae } \\
\hline Chaptalia nutans & Leaf & B. asper & - & Edema & [129] \\
\hline $\begin{array}{l}\text { Eclipta prostrata (syn. Eclipta } \\
\text { alba) }\end{array}$ & Aerial parts & $\begin{array}{l}\text { B. jararaca, } B . \\
\text { jararacussu }\end{array}$ & Myotoxicity & Myotoxicity & {$[130]$} \\
\hline Mikania glomerata & Leaf & B. jararaca & - & $\begin{array}{l}\text { Edema*, } \\
\text { hemorrhage*, }^{*} \\
\text { peritonitis }\end{array}$ & {$[131,132]$} \\
\hline Neurolaena lobata $a^{\#}$ & $\begin{array}{l}\text { Branch, leaf, } \\
\text { stem }\end{array}$ & B. atrox & - & Hemorrhage* $^{*}$ & {$[124]$} \\
\hline Pseudelephantopus spicatus & Whole plant & B. atrox & - & Hemorrhage* $^{*}$ & {$[124]$} \\
\hline \multicolumn{6}{|l|}{ Bignoniaceae } \\
\hline $\begin{array}{l}\text { Fridericia chica (syn. } \\
\text { Arrabidaea chica) }\end{array}$ & Leaf & B. atrox & - & Edema & {$[133]$} \\
\hline Tabebuia aurea & Stem bark & B. neuwiedi & $\begin{array}{c}\mathrm{H}_{2} \mathrm{O}_{2} \text { production by } \\
\text { peritoneal } \\
\text { macrophages }\end{array}$ & $\begin{array}{c}\text { Edema, hemorrhage* } \\
\text { myonecrosis*, } \\
\text { peritonitis }\end{array}$ & {$[110]$} \\
\hline Tabebuia rosea ${ }^{\#}$ & Stem bark & B. asper, B. atrox & $\mathrm{PLA}_{2}$ & $\begin{array}{c}\text { Edema }^{* *} \\
\text { Hemorrhage }^{*}\end{array}$ & {$[124,127,134]$} \\
\hline \multicolumn{6}{|l|}{ Bixaceae } \\
\hline Bixa orellana & Branch, leaf & B. asper, B. atrox & $\mathrm{PLA}_{2}$ & $\begin{array}{c}\text { Edema }^{* *} \\
\text { hemorrhage }^{*}\end{array}$ & {$[124,127,134]$} \\
\hline \multicolumn{6}{|l|}{ Boraginaceae } \\
\hline Cordia verbenacea & Leaf & B. jararacussu & - & Edema* & {$[135]$} \\
\hline \multicolumn{6}{|l|}{ Clusiaceae } \\
\hline Clusia fluminensis & Fruit & B. jararaca & Proteolytic & Hemorrhage $^{* *}$ & {$[136]$} \\
\hline \multicolumn{6}{|l|}{ Combretaceae } \\
\hline Combretum leprosum & Root & $\begin{array}{l}\text { B. jararaca, } B . \\
\text { jararacussu }\end{array}$ & $\begin{array}{c}\text { Collagenase, } \\
\text { myotoxicity, PLA } \\
\text { proteolytic }\end{array}$ & $\begin{array}{l}\text { Edema, hemorrhage*, } \\
\text { myotoxicity }\end{array}$ & {$[99]$} \\
\hline
\end{tabular}


TABle 3: Continued.

\begin{tabular}{|c|c|c|c|c|c|}
\hline \multirow{2}{*}{ Plant name } & \multirow{2}{*}{ Part used } & \multirow{2}{*}{ Snake venom } & \multicolumn{2}{|c|}{ Inhibited activities } & \multirow{2}{*}{ Reference(s) } \\
\hline & & & In vitro & In vivo & \\
\hline \multicolumn{6}{|l|}{ Connaraceae } \\
\hline Connarus favosus & Bark & $\begin{array}{l}\text { B. atrox, } B \text {. } \\
\text { jararaca }\end{array}$ & $\mathrm{PLA}_{2}$, proteolytic & $\begin{array}{c}\text { Edema* }^{*} \\
\text { hemorrhage }^{* * *}\end{array}$ & {$[33,115,128]$} \\
\hline \multicolumn{6}{|l|}{ Costaceae } \\
\hline Costus lasius ${ }^{\#}$ & ND & B. atrox & $\mathrm{PLA}_{2}$ & - & {$[134]$} \\
\hline Costus spicatus & Leaf & B. atrox & - & $\begin{array}{c}\text { Edema* } \\
\text { hyperalgesia* }\end{array}$ & {$[137]$} \\
\hline \multicolumn{6}{|l|}{ Crassulaceae } \\
\hline $\begin{array}{l}\text { Bryophyllum pinnatum (syn. } \\
\text { Kalanchoe pinnata) }\end{array}$ & Leaf & B. jararaca & $\mathrm{PLA}_{2}$ & Edema, hemorrhage & {$[138]$} \\
\hline $\begin{array}{l}\text { Kalanchoe laciniata (syn. } \\
\text { Kalanchoe brasiliensis) }\end{array}$ & Leaf & B. jararaca & $\mathrm{PLA}_{2}$ & $\begin{array}{c}\text { Edema, } \\
\text { hemorrhage }^{* * *}\end{array}$ & {$[33,138]$} \\
\hline \multicolumn{6}{|l|}{ Dicranaceae } \\
\hline Dicranum frigidum & Whole plant & B. asper & $\mathrm{PLA}_{2}$ & - & {$[111]$} \\
\hline \multicolumn{6}{|l|}{ Dilleniaceae } \\
\hline Davilla elliptica & Leaf & B. jararaca & - & Hemorrhage* $^{*}$ & [139] \\
\hline \multicolumn{6}{|l|}{ Euphorbiaceae } \\
\hline Croton urucurana & Stem bark & B. jararaca & - & Hemorrhage $^{*}$ & {$[140]$} \\
\hline Hevea nitida & Leaf, twig & B. asper & $\mathrm{PLA}_{2}$ & - & {$[111]$} \\
\hline Jatropha gossypiifolia & Leaf & $\begin{array}{l}\text { B. erythromelas, } \\
\text { B. jararaca }\end{array}$ & $\begin{array}{l}\mathrm{PLA}_{2} \text {, proteolytic, } \\
\text { SVH }\end{array}$ & $\begin{array}{l}\text { Edema, hemorrhage, } \\
\text { myotoxicity }\end{array}$ & {$[96,119]$} \\
\hline Jatropha mollissima ${ }^{\#}$ & Leaf & $\begin{array}{l}\text { B. erythromelas, } \\
\text { B. jararaca }\end{array}$ & - & $\begin{array}{c}\text { Edema, hemorrhage, } \\
\text { myotoxicity, } \\
\text { peritonitis }\end{array}$ & {$[141]$} \\
\hline \multicolumn{6}{|l|}{ Fabaceae } \\
\hline Abarema cochliacarpos & Stem bark & B. leucurus & - & $\begin{array}{l}\text { Decreased motor } \\
\text { function, edema, } \\
\text { hyperalgesia, } \\
\text { myotoxicity }\end{array}$ & {$[101]$} \\
\hline Brownea ariza & Bark, leaf & B. asper & $\mathrm{PLA}_{2}$, proteolytic & - & {$[142]$} \\
\hline Brownea rosa-de-monte & Leaf, stem bark & B. asper, B. atrox & $\mathrm{PLA}_{2}$, proteolytic & $\begin{array}{c}\text { Edema }^{* * *} \\
\text { hemorrhage }^{* * *}\end{array}$ & {$[124,127,134,143]$} \\
\hline Cassia fistula & Seed & B. jararaca & - & Hemorrhage $^{*}$ & {$[33]$} \\
\hline Dipteryx alata & Bark & B. jararacussu & Myotoxicity & - & {$[144]$} \\
\hline Pentaclethra macroloba ${ }^{\#}$ & Bark & $\begin{array}{c}\text { B. alternatus, } B . \\
\text { asper, } B . \text { atrox, } \\
\text { B. jararaca, } B . \\
\text { jararacussu, } B . \\
\text { moojeni, } B . \\
\text { neuwiedi, } B . \\
\text { pirajai }\end{array}$ & $\mathrm{PLA}_{2}$ & $\begin{array}{l}\text { Edema*, } \\
\text { hemorrhage*, }^{*} \\
\text { myotoxicity }^{*}\end{array}$ & {$[145]$} \\
\hline Plathymenia reticulata ${ }^{\#}$ & Bark & $\begin{array}{l}\text { B. atrox, } B \text {. } \\
\text { jararaca }\end{array}$ & $\mathrm{PLA}_{2}$ & $\begin{array}{c}\text { Edema*, } \\
\text { hemorrhage }^{*}\end{array}$ & {$[33,128]$} \\
\hline Schizolobium parahyba & Leaf & $\begin{array}{l}\text { B. alternatus, } B . \\
\text { moojeni, } B . \\
\text { pauloensis }\end{array}$ & $\mathrm{PLA}_{2}$ & $\begin{array}{l}\text { Hemorrhage*, } \\
\text { myotoxicity*** }\end{array}$ & {$[146,147]$} \\
\hline Senna dariensis & Whole plant & B. atrox & $\mathrm{PLA}_{2}$ & Hemorrhage* & {$[124,134]$} \\
\hline \multicolumn{6}{|l|}{ Heliconiaceae } \\
\hline Heliconia curtispatha & Rhizome & B. asper, B. atrox & $\mathrm{PLA}_{2}$, proteolytic & $\begin{array}{l}\text { Edema }^{* * *} \\
\text { hemorrhage }^{*}\end{array}$ & {$[124,127,134,148]$} \\
\hline Heliconia latispatha & Rhizome & B. asper & $\mathrm{PLA}_{2}$, proteolytic & - & {$[148]$} \\
\hline Heliconia wagneriana & Rhizome & B. asper & $\mathrm{PLA}_{2}$, proteolytic & - & {$[148]$} \\
\hline
\end{tabular}


TABle 3: Continued.

\begin{tabular}{|c|c|c|c|c|c|}
\hline \multirow{2}{*}{ Plant name } & \multirow{2}{*}{ Part used } & \multirow{2}{*}{ Snake venom } & \multicolumn{2}{|c|}{ Inhibited activities } & \multirow{2}{*}{ Reference(s) } \\
\hline & & & In vitro & In vivo & \\
\hline \multicolumn{6}{|l|}{ Hymenophyllaceae } \\
\hline Trichomanes elegans ${ }^{\#}$ & Whole plant & B. asper, B. atrox & $\mathrm{PLA}_{2}$ & $\begin{array}{l}\text { Edema }^{* * *} \\
\text { hemorrhage }^{*}\end{array}$ & {$[124,127,134]$} \\
\hline \multicolumn{6}{|l|}{ Hypericaceae } \\
\hline Hypericum brasiliense & Whole plant & B. jararaca & Proteolytic & $\begin{array}{l}\text { Edema* }^{*} \\
\text { hemorrhage* }\end{array}$ & [149] \\
\hline \multicolumn{6}{|l|}{ Icacinaceae } \\
\hline $\begin{array}{l}\text { Casimirella ampla (syn. } \\
\text { Humirianthera ampla) }\end{array}$ & Root & $\begin{array}{l}\text { B. atrox, } B . \\
\text { jararaca, } B . \\
\text { jararacussu }\end{array}$ & $\begin{array}{l}\text { Myotoxicity, PLA2, } \\
\text { proteolytic }\end{array}$ & $\begin{array}{l}\text { Edema***, } \\
\text { hemorrhage }^{* *}, \\
\text { myotoxicity }^{*}\end{array}$ & {$[102]$} \\
\hline \multicolumn{6}{|l|}{ Lamiaceae } \\
\hline Marsypianthes chamaedrys ${ }^{\#}$ & $\begin{array}{l}\text { Inflorescence, } \\
\text { leaf }\end{array}$ & B. atrox & $\mathrm{PLA}_{2}$ & Peritonitis & {$[108]$} \\
\hline Peltodon radicans & $\begin{array}{l}\text { Flower, leaf, } \\
\text { stem }\end{array}$ & B. atrox & - & Edema & {$[150]$} \\
\hline \multicolumn{6}{|l|}{ Lauraceae } \\
\hline $\begin{array}{l}\text { Aniba parviflora (syn. Aniba } \\
\text { fragrans) }^{\#}\end{array}$ & Bark, leaf & $\begin{array}{l}\text { B. atrox, } B \text {. } \\
\text { jararaca }\end{array}$ & $\mathrm{PLA}_{2}$ & $\begin{array}{c}\text { Edema*, } \\
\text { hemorrhage* }\end{array}$ & {$[33,128]$} \\
\hline \multicolumn{6}{|l|}{ Loasaceae } \\
\hline $\begin{array}{l}\text { Nasa speciosa (syn. Loasa } \\
\text { speciosa) }\end{array}$ & Leaf & B. asper & - & Edema & [129] \\
\hline \multicolumn{6}{|l|}{ Loganiaceae } \\
\hline Strychnos pseudoquina & Leaf & B. jararaca & - & Hemorrhage* & [139] \\
\hline Strychnos xinguensis ${ }^{\#}$ & $\mathrm{ND}$ & B. atrox & $\mathrm{PLA}_{2}$ & - & {$[134]$} \\
\hline \multicolumn{6}{|l|}{ Loranthaceae } \\
\hline Struthanthus orbicularis ${ }^{\#}$ & Branch, leaf & B. asper, B. atrox & $\mathrm{PLA}_{2}$ & $\begin{array}{c}\text { Edema }^{* *} \\
\text { hemorrhage }^{*}\end{array}$ & {$[124,127,134]$} \\
\hline \multicolumn{6}{|l|}{ Magnoliaceae } \\
\hline $\begin{array}{l}\text { Magnolia espinalii (syn. } \\
\text { Talauma espinalii) }\end{array}$ & Leaf, twig & B. asper & $\mathrm{PLA}_{2}$ & - & {$[111]$} \\
\hline $\begin{array}{l}\text { Magnolia guatapensis (syn. } \\
\text { Dugandiodendron guatapense) }\end{array}$ & Leaf, twig & B. asper & $\mathrm{PLA}_{2}$ & - & {$[111]$} \\
\hline $\begin{array}{l}\text { Magnolia hernandezii (syn. } \\
\text { Talauma hernandezii) }\end{array}$ & Leaf, twig & B. asper & $\mathrm{PLA}_{2}$ & - & {$[111]$} \\
\hline $\begin{array}{l}\text { Magnolia yarumalensis (syn. } \\
\text { Dugandiodendron yarumalense) }\end{array}$ & Leaf, twig & B. asper & $\mathrm{PLA}_{2}$ & - & {$[111]$} \\
\hline \multicolumn{6}{|l|}{ Malpighiaceae } \\
\hline Byrsonima crassa & Leaf & B. jararaca & - & Hemorrhage* & \\
\hline \multicolumn{6}{|l|}{ Malvaceae } \\
\hline $\begin{array}{l}\text { Pachira glabra (syn. } \\
\text { Bombacopsis glabra) }\end{array}$ & Root bark & B. pauloensis & - & Hemorrhage & {$[151]$} \\
\hline \multicolumn{6}{|l|}{ Melastomataceae } \\
\hline Bellucia dichotoma ${ }^{\#}$ & Bark & $\begin{array}{l}\text { B. atrox, } B . \\
\text { jararaca }\end{array}$ & $\mathrm{PLA}_{2}$ & $\begin{array}{c}\text { Edema }^{* * *} \\
\text { hemorrhage }^{* *}\end{array}$ & {$[33,98,128,152]$} \\
\hline Mouriri pusa & Leaf & B. jararaca & - & Hemorrhage* & {$[139]$} \\
\hline \multicolumn{6}{|l|}{ Meliaceae } \\
\hline Carapa guianensis & Leaf, twig & B. asper & $\mathrm{PLA}_{2}$ & - & {$[111]$} \\
\hline Cedrela odorata & Leaf, twig & B. asper & $\mathrm{PLA}_{2}$ & - & {$[111]$} \\
\hline Swietenia humilis & Leaf, twig & B. asper & $\mathrm{PLA}_{2}$ & - & {$[111]$} \\
\hline Swietenia macrophylla & Leaf, twig & B. asper & $\mathrm{PLA}_{2}$ & - & {$[111]$} \\
\hline Swietenia mahagoni & Leaf, twig & B. asper & $\mathrm{PLA}_{2}$ & - & [111] \\
\hline
\end{tabular}


TABLE 3: Continued.

\begin{tabular}{|c|c|c|c|c|c|}
\hline \multirow{2}{*}{ Plant name } & \multirow{2}{*}{ Part used } & \multirow{2}{*}{ Snake venom } & \multicolumn{2}{|c|}{ Inhibited activities } & \multirow{2}{*}{ Reference(s) } \\
\hline & & & In vitro & In vivo & \\
\hline \multicolumn{6}{|l|}{ Menispermaceae } \\
\hline Cissampelos pareira ${ }^{\#}$ & Leaf & B. asper & - & Hemorrhage $^{*}$ & {$[153]$} \\
\hline \multicolumn{6}{|l|}{ Moraceae } \\
\hline Brosimum guianense & Leaf & B. atrox & - & Hemorrhage*, pain* & {$[154]$} \\
\hline Castilla elastica ${ }^{\#}$ & $\begin{array}{l}\text { Branch, leaf, } \\
\text { stem }\end{array}$ & B. atrox & - & Hemorrhage* $^{*}$ & {$[124]$} \\
\hline Ficus nymphaeifolia & $\begin{array}{l}\text { Branch, leaf, } \\
\text { stem }\end{array}$ & B. asper, B. atrox & - & $\begin{array}{c}\text { Edema** }^{* *} \\
\text { hemorrhage }^{*}\end{array}$ & {$[124,127]$} \\
\hline \multicolumn{6}{|l|}{ Musaceae } \\
\hline Musa $\times$ paradisíaca ${ }^{\#}$ & Exudate & B. jararacussu & $\mathrm{PLA}_{2}$ & $\begin{array}{l}\text { Hemorrhage } \\
\text { myonecrosis }^{* *}\end{array}$ & {$[155]$} \\
\hline \multicolumn{6}{|l|}{ Myrtaceae } \\
\hline Myrcia guianensis & Leaf & B. jararaca & $\mathrm{PLA}_{2}$ & Hemorrhage $^{*}$ & {$[156]$} \\
\hline \multicolumn{6}{|l|}{ Passifloraceae } \\
\hline Passiflora quadrangularis ${ }^{\#}$ & Branch, leaf & B. atrox & - & Hemorrhage* & {$[124]$} \\
\hline \multicolumn{6}{|l|}{ Piperaceae } \\
\hline Piper arboreum ${ }^{\#}$ & Branch, leaf & B. atrox & $\mathrm{PLA}_{2}$ & Hemorrhage* & {$[124,134]$} \\
\hline Piper pulchrum ${ }^{\#}$ & $\begin{array}{l}\text { Leaf, branch, } \\
\text { stem }\end{array}$ & B. atrox & - & Hemorrhage $^{*}$ & {$[124]$} \\
\hline \multicolumn{6}{|l|}{ Polypodiaceae } \\
\hline Pleopeltis percussa $a^{\#}$ & $\begin{array}{l}\text { Branch, leaf, } \\
\text { stem, whole } \\
\text { plant }\end{array}$ & B. asper, B. atrox & $\mathrm{PLA}_{2}$, proteolytic & $\begin{array}{c}\text { Edema** }^{* *} \\
\text { hemorrhage }^{*}\end{array}$ & {$[124,127,134]$} \\
\hline \multicolumn{6}{|l|}{ Rubiaceae } \\
\hline Gonzalagunia panamensis & $\begin{array}{l}\text { Branch, leaf, } \\
\text { stem }\end{array}$ & B. asper, B. atrox & $\mathrm{PLA}_{2}$ & $\begin{array}{c}\text { Edema }^{* *} \\
\text { hemorrhage }^{*}\end{array}$ & {$[124,127,134]$} \\
\hline Randia aculeata $a^{\#}$ & Fruit & B. asper & - & Myotoxicity & {$[78]$} \\
\hline Uncaria tomentosa & Root & B. asper & - & Edema & {$[129]$} \\
\hline \multicolumn{6}{|l|}{ Rutaceae } \\
\hline Citrus limon & Ripe fruit & B. asper, B. atrox & - & $\begin{array}{l}\text { Edema }^{* * *} \\
\text { hemorrhage }^{*}\end{array}$ & {$[124,127]$} \\
\hline Murraya paniculata & Leaf, twig & B. asper & $\mathrm{PLA}_{2}$ & - & {$[111]$} \\
\hline \multicolumn{6}{|l|}{ Salicaceae } \\
\hline Casearia grandiflora ${ }^{\#}$ & Leaf & $\begin{array}{l}\text { B. moojeni, } B . \\
\text { neuwiedi }\end{array}$ & $\mathrm{PLA}_{2}$ & Myotoxicity ${ }^{*}$ & {$[157]$} \\
\hline Casearia sylvestris ${ }^{\#}$ & Leaf & $\begin{array}{c}\text { B. asper, } B . \\
\text { jararacussu, } B \text {. } \\
\text { moojeni, } B . \\
\text { neuwiedi, } B . \\
\text { pirajai }\end{array}$ & $\begin{array}{l}\text { Myonecrosis, } \\
\text { neuromuscular } \\
\text { blockade }\end{array}$ & $\begin{array}{c}\text { Edema*, } \\
\text { hemorrhage*, } \\
\text { myotoxicity* }\end{array}$ & {$[158-160]$} \\
\hline \multicolumn{6}{|l|}{ Sapindaceae } \\
\hline Billia hippocastanum & Leaf, twig & B. asper & $\mathrm{PLA}_{2}$ & - & {$[111]$} \\
\hline Cupania americana & Leaf, twig & B. asper & $\mathrm{PLA}_{2}$ & - & {$[111]$} \\
\hline Sapindus saponaria & $\begin{array}{c}\text { In vitro } \\
\text { cultivated callus, } \\
\text { leaf, twig }\end{array}$ & $\begin{array}{l}\text { B. alternatus, } B . \\
\text { asper, } B . \\
\text { jararacussu, } B . \\
\text { moojeni }\end{array}$ & $\mathrm{PLA}_{2}$ & Hemorrhage $^{*}$ & {$[111,161]$} \\
\hline Serjania erecta & Aerial parts & B. jararacussu & $\mathrm{PLA}_{2}$ & $\begin{array}{c}\text { Edema }^{*}, \\
\text { hemorrhage*, } \\
\text { myotoxicity* }\end{array}$ & {$[162]$} \\
\hline
\end{tabular}


TABLE 3: Continued.

\begin{tabular}{|c|c|c|c|c|c|}
\hline \multirow{2}{*}{ Plant name } & \multirow{2}{*}{ Part used } & \multirow{2}{*}{ Snake venom } & \multicolumn{2}{|c|}{ Inhibited activities } & \multirow{2}{*}{ Reference(s) } \\
\hline & & & In vitro & In vivo & \\
\hline \multicolumn{6}{|l|}{ Siparunaceae } \\
\hline Siparuna thecaphora & $\begin{array}{l}\text { Branch, leaf, } \\
\text { stem }\end{array}$ & B. atrox & - & Hemorrhage* $^{*}$ & {$[124]$} \\
\hline \multicolumn{6}{|l|}{ Solanaceae } \\
\hline $\begin{array}{l}\text { Capsicum annuum (syn. } \\
\text { Capsicum frutescens) }{ }^{\#}\end{array}$ & Ripe fruit & B. atrox & - & Hemorrhage $^{*}$ & {$[124]$} \\
\hline \multicolumn{6}{|l|}{ Urticaceae } \\
\hline Urera baccifera & Leaf & B. asper & - & Edema & [129] \\
\hline \multicolumn{6}{|l|}{ Velloziaceae } \\
\hline $\begin{array}{l}\text { Vellozia squamata (syn. Vellozia } \\
\text { flavicans) }\end{array}$ & Leaf & B. jararacussu & $\begin{array}{l}\text { Neuromuscular } \\
\text { blockade and cell } \\
\text { damage }\end{array}$ & - & {$[163]$} \\
\hline \multicolumn{6}{|l|}{ Zingiberaceae } \\
\hline Curcuma longa & Rhizome & B. alternatus & - & $\begin{array}{l}\text { Edema, hemorrhage, } \\
\text { necrosis }\end{array}$ & {$[164]$} \\
\hline Renealmia alpinia ${ }^{\#}$ & Leaf, rhizome & B. asper, B. atrox & $\mathrm{PLA}_{2}$, proteolytic & $\begin{array}{c}\text { Edema }^{* *}, \\
\text { hemorrhage }\end{array}$ & {$[107,127,134,165,166]$} \\
\hline
\end{tabular}

cardiotoxicity, respiratory and circulatory collapse, necrosis, hemorrhage, and edema [13]. A great number of the plants showed in this review were tested against Naja species. However, it is important to mention that only a very small number of these plants were assessed in vivo, and so the scientific evidences of antiophidic activities of these species are based on enzymatic in vitro assays, especially against SVHs, a class of toxin particularly relevant in cobras. The study of Molander et al. [82] presented several medicinal plants identified as potent inhibitors of $\mathrm{N}$. nigricollis SVHs, $\mathrm{PLA}_{2}$, and proteases, which could indicate a potential rich source of inhibitors of necrosis induced by these venom, which must be evaluated in vivo later [82]. The same group, in a more recent study [123], investigated the skin permeation, ex vivo inhibition of venom induced tissue destruction, and wound healing potential of African plants used against snakebite, which included the most potent inhibitors identified in the previous work [82]. A total of 30 plant species were tested against Naja nigricollis and Bitis arietans employing in vitro and ex vivo models [123]. However, although plant extracts have showed potential in inhibiting snake venom enzymes, this study showed no effect against cell death and tissue damage.

4.3. Plants Inhibiting Bothrops Snakes. A summary of active plants against Bothrops snakes local effects is presented in Table 3. More than $90 \%$ of the snakebites reported every year in Latin America are caused by Bothrops species [8]. Envenomation by Bothrops snakes is characterized by a prominent and complex series of local pathological alterations, which appear rapidly after the bite in the anatomical site where venom is inoculated [168]. In a number of Bothrops bite cases, lack of neutralization of local effects results in permanent sequelae, with significative tissue loss [8]. So, the use of a therapeutic approach with high inhibitory potential and easy access and disponibility to victims, which could neutralize rapidly the onset of these local manifestations, is interesting. Most of the inhibitory studies with Bothrops snakes were performed in Brazil, which could be associated with richness of Brazilian flora as well as the epidemiological aspects of this country. The work performed by De Moura et al. [33] could be highlighted, where these authors performed an ethnopharmacological-guided screening of plants with reputation against snakebite in Santarém, Western Pará, Brazil. Twelve species were evaluated against Bothrops jararaca snake venom induced hemorrhage and some of them presented very significative results, showing, thus, the relevance of traditional knowledge in the survey of antiophidic plants [33].

4.4. Plants Inhibiting Bitis Snakes. A summary of active plants against Bitis snakes local effects is presented in Table 4. Snakes belonging to the genus Bitis are implicated in many accidents with humans in Africa. The envenomation by Bitis often results in severe local damage, hypotension, coagulopathy, thrombocytopenia, and spontaneous local bleeding and, in the absence of antivenom therapy, the accident can be fatal. Bitis arietans is one of the three species of snakes of medical importance in Africa and its venom is considered the most 
TABLE 4: List of medicinal plants with inhibitory potential against local effects induced by Bitis snakes.

\begin{tabular}{|c|c|c|c|c|c|}
\hline \multirow{2}{*}{ Plant name } & \multirow{2}{*}{ Part used } & \multirow{2}{*}{ Snake venom } & \multicolumn{2}{|c|}{ Inhibited activities } & \multirow{2}{*}{ Reference(s) } \\
\hline & & & In vitro & In vivo & \\
\hline \multicolumn{6}{|l|}{ Amaranthaceae } \\
\hline Pupalia lappacea & Herbal & B. arietans & SVH & - & {$[82]$} \\
\hline \multicolumn{6}{|l|}{ Amaryllidaceae } \\
\hline Crinum jagus & Bulb & B. arietans & - & Myotoxicity $^{*}$ & {$[167]$} \\
\hline \multicolumn{6}{|l|}{ Anacardiaceae } \\
\hline Lannea acida & Cortex & B. arietans & $\mathrm{PLA}_{2}$, proteolytic, SVH & - & {$[82]$} \\
\hline Sclerocarya birrea & Cortex & B. arietans & $\mathrm{PLA}_{2}$, proteolytic, SVH & - & {$[82]$} \\
\hline Spondias mombin ${ }^{\#}$ & Cortex, radix & B. arietans & $\mathrm{PLA}_{2}$, proteolytic, SVH & - & {$[82]$} \\
\hline \multicolumn{6}{|l|}{ Annonaceae } \\
\hline Annona senegalensis ${ }^{\#}$ & Cortex & B. arietans & $\mathrm{PLA}_{2}$, proteolytic, SVH & - & {$[82]$} \\
\hline \multicolumn{6}{|l|}{ Apocynaceae } \\
\hline Strophanthus speciosus & Radix & B. arietans & SVH & - & {$[82]$} \\
\hline \multicolumn{6}{|l|}{ Araliaceae } \\
\hline Polyscias fulva & Cortex & B. arietans & SVH & - & {$[82]$} \\
\hline \multicolumn{6}{|l|}{ Bignoniaceae } \\
\hline Kigelia africana & Cortex & B. arietans & $\mathrm{PLA}_{2}, \mathrm{SVH}$ & - & {$[82]$} \\
\hline \multicolumn{6}{|l|}{ Bixaceae } \\
\hline $\begin{array}{l}\text { Cochlospermum tinctorium } \\
\text { Capparaceae }\end{array}$ & Radix & B. arietans & $\mathrm{PLA}_{2}$, proteolytic, SVH & - & {$[82]$} \\
\hline Capparis tomentosa & Radix & B. arietans & $\mathrm{PLA}_{2}$, proteolytic, SVH & - & {$[82]$} \\
\hline \multicolumn{6}{|l|}{ Colchicaceae } \\
\hline Gloriosa superba & Radix & B. arietans & $\mathrm{SVH}$ & - & {$[82]$} \\
\hline \multicolumn{6}{|l|}{ Combretaceae } \\
\hline Combretum molle & Folium & B. arietans & $\mathrm{PLA}_{2}$, proteolytic, SVH & - & {$[82]$} \\
\hline Guiera senegalensis & Radix & B. arietans & $\mathrm{PLA}_{2}$, proteolytic, $\mathrm{SVH}$ & - & {$[82]$} \\
\hline \multicolumn{6}{|l|}{ Ebenaceae } \\
\hline Diospyros mespiliformis & Cortex & B. arietans & $\mathrm{PLA}_{2}$, proteolytic, SVH & - & {$[82]$} \\
\hline \multicolumn{6}{|l|}{ Euphorbiaceae } \\
\hline Alchornea laxiflora & Cortex & B. arietans & $\mathrm{PLA}_{2}$, proteolytic, SVH & - & {$[82]$} \\
\hline \multicolumn{6}{|l|}{ Fabaceae } \\
\hline Bauhinia thonningii & Cortex, radix & B. arietans & $\mathrm{PLA}_{2}$, proteolytic, SVH & - & {$[82]$} \\
\hline $\begin{array}{l}\text { Bobgunnia madagascariensis } \\
\text { (syn. Swartzia madagascariensis) }\end{array}$ & Folium, radix & B. arietans & $\mathrm{PLA}_{2}$, proteolytic, $\mathrm{SVH}$ & - & {$[82]$} \\
\hline Burkea africana & Cortex & B. arietans & $\mathrm{PLA}_{2}$, proteolytic, SVH & - & {$[82]$} \\
\hline Dichrostachys cinerea & Folium & B. arietans & $\mathrm{PLA}_{2}$, proteolytic, $\mathrm{SVH}$ & - & {$[82]$} \\
\hline Entada africana & Radix & B. arietans & SVH & - & {$[82]$} \\
\hline Parkia biglobosa & Cortex & B. arietans & $\mathrm{PLA}_{2}$, proteolytic, SVH & - & {$[82]$} \\
\hline Stylosanthes erecta & Folium & B. arietans & $\mathrm{SVH}$ & - & {$[82]$} \\
\hline Tamarindus indica ${ }^{\#}$ & Cortex, folium & B. arietans & $\mathrm{PLA}_{2}$, proteolytic, $\mathrm{SVH}$ & - & {$[82]$} \\
\hline \multicolumn{6}{|l|}{ Hypericaceae } \\
\hline Psorospermum corymbiferum & Cortex, radix & B. arietans & $\mathrm{PLA}_{2}$, proteolytic, $\mathrm{SVH}$ & - & {$[82]$} \\
\hline \multicolumn{6}{|l|}{ Hypoxidaceae } \\
\hline $\begin{array}{l}\text { Molineria capitulata (syn. } \\
\text { Curculigo recurvata) }\end{array}$ & Folium & B. arietans & $\mathrm{SVH}$ & - & {$[82]$} \\
\hline
\end{tabular}


TABle 4: Continued.

\begin{tabular}{|c|c|c|c|c|c|}
\hline \multirow{2}{*}{ Plant name } & \multirow{2}{*}{ Part used } & \multirow{2}{*}{ Snake venom } & \multicolumn{2}{|c|}{ Inhibited activities } & \multirow{2}{*}{ Reference(s) } \\
\hline & & & In vitro & In vivo & \\
\hline \multicolumn{6}{|l|}{ Lamiaceae } \\
\hline $\begin{array}{l}\text { Rotheca myricoides (syn. } \\
\text { Clerodendrum myricoides) }\end{array}$ & Cortex & B. arietans & SVH & - & {$[82]$} \\
\hline Teucrium kraussii & $\begin{array}{l}\text { Aerial parts, } \\
\text { cortex }\end{array}$ & B. arietans & SVH & - & {$[82]$} \\
\hline $\begin{array}{l}\text { Volkameria glabra (syn. } \\
\text { Clerodendrum glabrum) }\end{array}$ & Cortex & B. arietans & $\mathrm{PLA}_{2}$, proteolytic, SVH & - & {$[82]$} \\
\hline \multicolumn{6}{|l|}{ Lauraceae } \\
\hline Cassytha filiformis & Herbal & B. arietans & SVH & - & {$[82]$} \\
\hline \multicolumn{6}{|l|}{ Loganiaceae } \\
\hline Strychnos decussata & Radix & B. arietans & Proteolytic & - & {$[82]$} \\
\hline Strychnos innocua & Folium & B. arietans & Proteolytic, SVH & - & {$[82]$} \\
\hline \multicolumn{6}{|l|}{ Malvaceae } \\
\hline Dombeya quinqueseta & Cortex & B. arietans & $\mathrm{PLA}_{2}$, proteolytic, $\mathrm{SVH}$ & - & {$[82]$} \\
\hline Grewia mollis & $\begin{array}{l}\text { Cortex, folium, } \\
\text { radix }\end{array}$ & B. arietans & $\mathrm{PLA}_{2}$, proteolytic, SVH & - & {$[82]$} \\
\hline Sterculia setigera & Cortex & B. arietans & $\mathrm{PLA}_{2}, \mathrm{SVH}$ & - & {$[82]$} \\
\hline Waltheria indica & Radix & B. arietans & $\mathrm{PLA}_{2}$, proteolytic, SVH & - & {$[82]$} \\
\hline \multicolumn{6}{|l|}{ Menispermaceae } \\
\hline $\begin{array}{l}\text { Cissampelos mucronata } \\
\text { Moraceae }\end{array}$ & \multicolumn{4}{|c|}{ Moraceae } & {$[82]$} \\
\hline Ficus platyphylla & Folium & B. arietans & $\mathrm{PLA}_{2}, \mathrm{SVH}$ & - & {$[82]$} \\
\hline \multicolumn{6}{|l|}{ Olacaceae } \\
\hline Ximenia americana & Folium & B. arietans & $\mathrm{PLA}_{2}$, proteolytic, SVH & - & {$[82]$} \\
\hline \multicolumn{6}{|l|}{ Phyllanthaceae } \\
\hline $\begin{array}{l}\text { Flueggea virosa (syn. Securinega } \\
\text { virosa) }\end{array}$ & Radix & B. arietans & $\mathrm{PLA}_{2}$, proteolytic, SVH & - & {$[82]$} \\
\hline \multicolumn{6}{|l|}{ Primulaceae } \\
\hline Maesa lanceolata & Cortex & B. arietans & $\mathrm{PLA}_{2}$, proteolytic, $\mathrm{SVH}$ & - & {$[82]$} \\
\hline \multicolumn{6}{|l|}{ Rhamnaceae } \\
\hline Ziziphus mucronata & Radix & B. arietans & $\mathrm{PLA}_{2}$, proteolytic, SVH & - & {$[82]$} \\
\hline Ziziphus spina-christi & Cortex & B. arietans & $\mathrm{PLA}_{2}$, proteolytic, SVH & - & {$[82]$} \\
\hline \multicolumn{6}{|l|}{ Rubiaceae } \\
\hline Crossopteryx febrifuga & Cortex & B. arietans & $\mathrm{PLA}_{2}, \mathrm{SVH}$ & - & {$[82]$} \\
\hline Pentanisia prunelloides & Radix & B. arietans & $\mathrm{PLA}_{2}$, proteolytic, $\mathrm{SVH}$ & - & {$[82]$} \\
\hline Pentas zanzibarica & Folium & B. arietans & $\mathrm{PLA}_{2}$ & - & {$[82]$} \\
\hline \multicolumn{6}{|l|}{ Rutaceae } \\
\hline Zanthoxylum capense & Radix & B. arietans & $\mathrm{PLA}_{2}$, proteolytic & - & {$[82]$} \\
\hline \multicolumn{6}{|l|}{ Sapindaceae } \\
\hline \multicolumn{5}{|l|}{ Solanaceae } & {$[82]$} \\
\hline Schwenckia americana & Folium & B. arietans & SVH & - & {$[82]$} \\
\hline \multicolumn{6}{|l|}{ Verbenaceae } \\
\hline Lantana trifolia & Cortex & B. arietans & $\mathrm{PLA}_{2}, \mathrm{SVH}$ & - & {$[82]$} \\
\hline \multicolumn{6}{|l|}{ Vitaceae } \\
\hline Cissus populnea & Stem & B. arietans & SVH & - & {$[82]$} \\
\hline
\end{tabular}

$\mathrm{PLA}_{2}=$ snake venom phospholipase $\mathrm{A}_{2} ; \mathrm{SVH}=$ snake venom hyaluronidase. ${ }^{\#}$ Vegetal species with related folk use as antiophidic agents, as showed in Table 1. ${ }^{*}$ Studies where inhibitory activity was assessed only by preincubation of venom with extract (see Section 4.1 for details). 
toxic venom of the viper group [169]. Regarding the plants with inhibitory action upon Bitis snakes, only one in vivo study of antiophidic activity was found until date. Although many works have been showing the potential of medicinal plants against several snake venoms, only three works were identified evaluating the action of plants against Bitis, from which two are the same screening studies of plants against Naja snake venom discussed before (Section 4.2) [82, 123].

4.5. Plants Inhibiting Daboia/Vipera Snakes. A summary of active plants against Daboia/Vipera snakes local effects is presented in Table 5. The Daboia genus is represented by a single species, named Daboia russelii, also popularly known as Russell's viper. This species is widespread in many parts of Asia and is responsible for large morbimortality due to snakebites in this continent $[183,184]$. Russell's viper was formerly classified in Vipera genus and is therefore better known as Vipera russelii, since the new accepted nomenclature (Daboia russelii) is not yet universally followed [184]. For this reason, to avoid confounding, we use the term Daboia/Vipera in some occasions.

In humans, Russell's viper bite causes severe local tissue damage; more frequently the necrosis results in an irreversible loss of tissue and requires amputation of the affected limb $[182,183,185]$. As observed with Bothrops snakes, several studies have showed the inhibitory potential of medicinal plants against local effects of Russell's viper venom, including several preclinical in vivo studies.

4.6. Plants Inhibiting Lachesis Snakes. A summary of active plants against Lachesis snakes local effects is presented in Table 6. Lachesis muta is the longest venomous snake in the Americas and is distributed in the equatorial forests east of the Andes, ranging from eastern Ecuador, Colombia, Peru, northern Bolivia, and eastern and northern Venezuela, to Guyana, French Guyana, Surinam, and northern Brazil [100, 186]. L. muta snakebites are mainly characterized by systemic (generalized bleeding, coagulopathy, renal failure, and shock) and local effects (pain, hemorrhage, edema, and necrosis). In South America, Bothrops species has a higher incidence of accidents than L. muta, but, on the other hand, Lachesis bites led to more severe symptoms and have lethality indexes significantly higher than Bothrops $[100,186,187]$. Thus, the study of medicinal plants against these snakes, too, is of very much relevance. However, only a few studies were detected with plants against Lachesis snakes.

4.7. Plants Inhibiting Crotalus Snakes. A summary of active plants against Crotalus snakes local effects is presented in Table 7. Snakes from Crotalus durissus complex, popularly known as rattlesnakes, are dispersed northward into North America and southward into South America. Species of the Crotalus durissus complex pose a serious medical problem in many parts of the America [199]. Crotalic venom is considered highly toxic and more lethal in comparison with that of the genus Bothrops, having three main actions: neurotoxic, myotoxic, and coagulant [200, 201]. The crotalic accident is characterized by local and systemic manifestations, but while the local alterations are only discrete, the systemic manifestations are severe, leading to high chances of death [201]. Probably due to this low local effect in envenomed victims, the inhibition of these effects by plants is, until now, little investigated, especially when compared to other species with characteristic severe local effects.

4.8. Plants Inhibiting Other Snakes. Besides the snakes discussed above, some other studies are found with plants inhibiting other snake species, such as those from Echis and Bungarus genus. For other snakes species such as Calloselasma rhodostoma, Philodryas olfersii, and Montivipera xanthina, only isolated studies with a single plant, in each one, were found. These plants are summarized in Table 8. Many reasons may be stated for this lack of studies, such as low level of local effects, incidence restricted to a small region of the world, and usual low efficacy of plant extracts due to possible extremely high toxicity. However, it is important to highlight that the lack of studies does not mean a lower medical relevance of these species. For example, the sawscaled viper (Echis carinatus) and the common Indian krait (Bungarus caeruleus), along with spectacled cobra (Naja naja) and Russell's viper (Daboia russelii), are included among the referred "Big Four" venomous snakes of India, being responsible for the majority of morbid complications, characterized by persistent and progressive tissue necrosis even after treatment with antivenom [195, 202]. Therefore, future studies with plants aiming at the inhibition of the local effects induced by these snakes are encouraged.

4.9. Studies in Humans. Along our antiophidic plants database, only one clinical study was found in literature, evaluating the inhibitory properties of a polyherbal formulation, externally applied, against soft-tissue necrosis after Naja atra (Chinese cobra) bite [203]. This polyherbal formulation, known in China as Jidesheng antivenom, is composed of the following ingredients: Ganchan (Succys Bufo), Dijincao (Herba Euphorbiae Humifusae), Chonglou (Rhizoma Paridis Chonglou), and Wugong (Scolopendra). This was a retrospective study performed with 126 patients with skin and softtissue necrosis due cobra bite, with the control group being treated externally with $40 \%$ glyceride magnesium sulfate $(n=52)$ and the treatment group performed by application of Jidesheng antivenom externally $(n=74)$. The authors observed statistically significant differences in maximum local necrotic area of skin and soft tissues, healing time, and skin-grafting rate between the control and treatment groups $(P<0.05)$, thus indicating that external application of Jidesheng antivenom may help to promote wound healing and reduce the skin-grafting rate in cases of skin and soft-tissue necrosis due to Chinese cobra bite [203]. Considering the composition of the Jidesheng antivenom, the authors discuss that each ingredient in this product may exert antipyretic, antidotal, antiphlogistic, and analgesic effects, according to previous results with each ingredient isolated, which could contribute to the inhibitory effect observed by the formulation [203]. The result obtained in this clinical study is very promising, since it shows that a plant-derived product 
TABLE 5: List of medicinal plants with inhibitory potential against local effects induced by Daboia/Vipera snakes.

\begin{tabular}{|c|c|c|c|c|c|}
\hline \multirow{2}{*}{ Plant name } & \multirow{2}{*}{ Part used } & \multirow{2}{*}{ Snake venom } & \multicolumn{2}{|c|}{ Inhibited activities } & \multirow{2}{*}{ Reference(s) } \\
\hline & & & In vitro & In vivo & \\
\hline \multicolumn{6}{|l|}{ Anacardiaceae } \\
\hline Anacardium occidentale & Bark & D. russelli & $\mathrm{PLA}_{2}$, proteolytic, $\mathrm{SVH}$ & $\begin{array}{c}\text { Edema, hemorrhage, } \\
\text { myotoxicity }\end{array}$ & {$[170]$} \\
\hline Mangifera indica ${ }^{\#}$ & Stem bark & D. russelii & $\begin{array}{l}\text { LAAO, } \mathrm{PLA}_{2}, \mathrm{SVH}, \\
\text { proteolytic }\end{array}$ & $\begin{array}{l}\text { Edema }^{*}, \text { hemorrhage }^{*}, \\
\text { myotoxicity }\end{array}$ & {$[171]$} \\
\hline \multicolumn{6}{|l|}{ Apocynaceae } \\
\hline Hemidesmus indicus ${ }^{\#}$ & Root & D. russelli & - & Hemorrhage & {$[172]$} \\
\hline Tylophora indica & Leaf, root & D. russelli & $\mathrm{PLA}_{2}$ & Hemorrhage & {$[85]$} \\
\hline \multicolumn{6}{|l|}{ Aristolochiaceae } \\
\hline Aristolochia bracteolata ${ }^{\#}$ & Leaf, root & D. russelli & $\mathrm{PLA}_{2}$ & Hemorrhage & {$[85]$} \\
\hline Aristolochia indica & Root & D. russelii & LAAO, proteolytic & - & {$[173]$} \\
\hline \multicolumn{6}{|l|}{ Asteraceae } \\
\hline Pluchea indica ${ }^{\#}$ & Root & D. russelli & - & Hemorrhage & {$[172]$} \\
\hline \multicolumn{6}{|l|}{ Euphorbiaceae } \\
\hline Acalypha indica & Leaf & D. r. russelli & - & Hemorrhage, necrosis & {$[174]$} \\
\hline \multicolumn{6}{|l|}{ Fabaceae } \\
\hline Butea monosperma ${ }^{\#}$ & Stem bark & D. russelii & SVH & Hemorrhage $^{*}$ & {$[175]$} \\
\hline Mimosa pudica & Root & D. russelii & Proteolytic, SVH & - & {$[91]$} \\
\hline Tamarindus indica ${ }^{\#}$ & Seed & $\begin{array}{l}\text { D. r. siamensis, } D . \\
\text { russelii }\end{array}$ & $\begin{array}{l}\text { LAAO, } \mathrm{PLA}_{2}, \mathrm{SVH}, \\
\text { proteolytic }\end{array}$ & $\begin{array}{l}\text { Edema* }^{*}, \text { hemorrhage }^{*}, \\
\text { myotoxicity }\end{array}$ & {$[176,177]$} \\
\hline \multicolumn{6}{|l|}{ Lamiaceae } \\
\hline Leucas aspera ${ }^{\#}$ & Leaf, root & D. russelii & $\mathrm{PLA}_{2}$ & Hemorrhage & {$[85]$} \\
\hline Vitex negundo ${ }^{\#}$ & Root & D. russelii & - & Edema, hemorrhage & {$[178]$} \\
\hline \multicolumn{6}{|l|}{ Loganiaceae } \\
\hline Strychnos nux-vomica & Seed & D. russelii & $\mathrm{PLA}_{2}$ & Hemorrhage $^{*}$ & {$[95]$} \\
\hline \multicolumn{6}{|l|}{ Moraceae } \\
\hline Morus alba & Leaf & D. russelii & Proteolytic, SVH & $\begin{array}{l}\text { Edema* }{ }^{*} \text {, hemorrhage* } \\
\text { myotoxicity }\end{array}$ & {$[179]$} \\
\hline \multicolumn{6}{|l|}{ Phyllanthaceae } \\
\hline $\begin{array}{l}\text { Phyllanthus emblica (syn. } \\
\text { Emblica officinalis) }\end{array}$ & Root & D. russelii & - & Edema, hemorrhage & {$[178]$} \\
\hline \multicolumn{6}{|l|}{ Piperaceae } \\
\hline Piper longum ${ }^{\#}$ & Fruit & D. russelii & Hemorrhage & $\begin{array}{l}\text { Edema, hemorrhage, } \\
\text { myotoxicity, necrosis }\end{array}$ & {$[104]$} \\
\hline \multicolumn{6}{|l|}{ Rubiaceae } \\
\hline Ophiorrhiza mungos ${ }^{\#}$ & Root & D. russelii & Hemorrhage & - & {$[180]$} \\
\hline \multicolumn{6}{|l|}{ Salvadoraceae } \\
\hline Azima tetracantha & Leaf & D. russelii & SVH & - & {$[181]$} \\
\hline \multicolumn{6}{|l|}{ Vitaceae } \\
\hline Vitis vinifera & Seed & D. russelii & Proteolytic, SVH & $\begin{array}{c}\text { Edema* }{ }^{*} \text {, hemorrhage* } \\
\text { myonecrosis }\end{array}$ & {$[182]$} \\
\hline
\end{tabular}

LAAO = L-amino acid oxidase $; \mathrm{PLA}_{2}=$ snake venom phospholipase $\mathrm{A}_{2} ; \mathrm{SVH}=$ snake venom hyaluronidase. ${ }^{\#}$ Vegetal species with related folk use as antiophidic agents, as showed in Table 1. *Studies where inhibitory activity was assessed only by preincubation of venom with extract (see Section 4.1 for details). 
TABLE 6: List of medicinal plants with inhibitory potential against local effects induced by Lachesis snakes.

\begin{tabular}{|c|c|c|c|c|c|}
\hline \multirow{2}{*}{ Plant name } & \multirow{2}{*}{ Part used } & \multirow{2}{*}{ Snake venom } & \multicolumn{2}{|c|}{ Inhibited activities } & \multirow{2}{*}{ Reference(s) } \\
\hline & & & In vitro & In vivo & \\
\hline \multicolumn{6}{|l|}{ Apocynaceae } \\
\hline $\begin{array}{l}\text { Fernaldia pandurata (syn. } \\
\text { Mandevilla velutina) }\end{array}$ & Root & L. muta & Proteolytic, $\mathrm{PLA}_{2}$ & Hemorrhage $^{*}$ & [188] \\
\hline \multicolumn{6}{|l|}{ Asteraceae } \\
\hline $\begin{array}{l}\text { Eclipta prostrata (syn. Eclipta } \\
\text { alba) }\end{array}$ & Aerial parts, root & L. muta & $\begin{array}{c}\text { Myotoxicity, } \\
\text { proteolytic, PLA }\end{array}$ & $\begin{array}{l}\text { Hemorrhage }^{*} \\
\text { myotoxicity }\end{array}$ & {$[130,188]$} \\
\hline Mikania glomerata & Root & L. muta & Proteolytic, $\mathrm{PLA}_{2}$ & - & {$[188]$} \\
\hline \multicolumn{6}{|l|}{ Erythroxylaceae } \\
\hline Erythroxylum ovalifolium & Stem & L. muta & Proteolytic, $\mathrm{PLA}_{2}$ & $\begin{array}{c}\text { Edema }^{* * *} \\
\text { hemorrhage }^{* * *}\end{array}$ & {$[189]$} \\
\hline Erythroxylum subsessile & Stem & L. muta & Proteolytic, $\mathrm{PLA}_{2}$ & $\begin{array}{c}\text { Edema }^{* * *} \\
\text { hemorrhage }^{* * *}\end{array}$ & {$[189]$} \\
\hline \multicolumn{6}{|l|}{ Euphorbiaceae } \\
\hline Jatropha elliptica & Root, stem & L. muta & Proteolytic, PLA 2 & Hemorrhage $^{*}$ & {$[188]$} \\
\hline \multicolumn{6}{|l|}{ Fabaceae } \\
\hline Pentaclethra macroloba ${ }^{\#}$ & Bark & L. muta & - & Hemorrhage $^{*}$ & {$[145]$} \\
\hline $\begin{array}{l}\text { Stryphnodendron adstringens } \\
\text { (syn. Stryphnodendron } \\
\text { barbatimam) }\end{array}$ & Root & L. muta & Proteolytic, $\mathrm{PLA}_{2}$ & Hemorrhage $^{*}$ & [188] \\
\hline \multicolumn{6}{|l|}{ Melastomataceae } \\
\hline Miconia albicans & Stem & L. muta & Proteolytic, PLA 2 & Hemorrhage* & {$[188]$} \\
\hline Miconia fallax & Stem & L. muta & Proteolytic, PLA 2 & Hemorrhage $^{*}$ & {$[188]$} \\
\hline Miconia sellowiana & ND & L. muta & Proteolytic, PLA 2 & Hemorrhage $^{*}$ & {$[188]$} \\
\hline Tibouchina stenocarpa & Root & L. muta & Proteolytic, $\mathrm{PLA}_{2}$ & Hemorrhage $^{*}$ & {$[188]$} \\
\hline \multicolumn{6}{|l|}{ Salicaceae } \\
\hline Casearia sylvestris ${ }^{\#}$ & Root & L. muta & Proteolytic & Hemorrhage $^{*}$ & {$[188]$} \\
\hline \multicolumn{6}{|l|}{ Sapotaceae } \\
\hline Manilkara subsericea & Leaf, stem & L. muta & Proteolytic, PLA 2 & $\begin{array}{c}\text { Edema }^{* *}, \\
\text { hemorrhage }^{* *}\end{array}$ & {$[100]$} \\
\hline
\end{tabular}

$\mathrm{ND}=$ information not described in the work; $\mathrm{PLA}_{2}=$ snake venom phospholipase $\mathrm{A}_{2} .{ }^{*}$ Vegetal species with related folk use as antiophidic agents, as showed in Table $1 .{ }^{*}$ Studies where inhibitory activity was assessed only by preincubation of venom with extract (see Section 4.1 for details). ${ }^{* *}$ Active in preincubation tests but inactive or only poorly active when extract was used independently of venom (pre-, co-, or posttreatment protocols). ${ }^{* * *}$ Active in preincubation tests and when used independently of venom (pre-, co-, or posttreatment protocols).

showed significant results in humans, thus pointing to the potentiality of this kind of product in treatment of snake venom induced local effects. However, only one study is insufficient to ensure the potentiality of medicinal plants against snakebites, with performing more clinical studies, preferentially controlled and randomized ones, to bring more evidences of the viability of the approach for future safe and effective use in humans being necessary. So, more clinical studies, especially ones with those plants highlighted in this review and those presenting good preclinical in vivo evidences of antiophidic efficacy, are highly encouraged.

\section{Concluding Remarks}

The popular use of vegetal species does not necessarily imply efficacy, but it gives a selected list of medicinal plants that can be primarily studied in pharmacologic assays for possible antiophidic effects, directing future studies in this area. In fact, a great number of these species that have been evaluated against local tissue damage induced by several snake species showed inhibitory potential against hyaluronidase, phospholipase, proteolytic, hemorrhagic, myotoxic, and edematogenic activities, among others. Therefore, considering the limitations of conventional antivenom serotherapy, especially 
TABLE 7: List of medicinal plants with inhibitory potential against local effects induced by Crotalus snakes.

\begin{tabular}{|c|c|c|c|c|c|}
\hline \multirow{2}{*}{ Plant name } & \multirow{2}{*}{ Part used } & \multirow{2}{*}{ Snake venom } & \multicolumn{2}{|c|}{ Inhibited activities } & \multirow{2}{*}{ Reference(s) } \\
\hline & & & In vitro & In vivo & \\
\hline \multicolumn{6}{|l|}{ Apocynaceae } \\
\hline $\begin{array}{l}\text { Fernaldia pandurata (syn. } \\
\text { Mandevilla velutina) }\end{array}$ & $\begin{array}{l}\text { Leaf, stem, } \\
\text { subterranean system }\end{array}$ & C. d. terrificus & $\mathrm{PLA}_{2}$ & $\begin{array}{l}\text { Edema*, } \\
\text { myotoxicity* }\end{array}$ & {$[125]$} \\
\hline Mandevilla illustris & Subterranean system & C.d. terrificus & $\mathrm{PLA}_{2}$ & - & {$[190]$} \\
\hline \multicolumn{6}{|l|}{ Asteraceae } \\
\hline Eclipta prostrata (syn. Eclipta alba) & Aerial parts & C. d. terrificus & Myotoxicity & Myotoxicity $^{*}$ & {$[11]$} \\
\hline \multicolumn{6}{|l|}{ Bignoniaceae } \\
\hline $\begin{array}{l}\text { Fridericia chica (syn. Arrabidaea } \\
\text { chica) }\end{array}$ & Leaf & C. d. ruruima & - & Edema & {$[133]$} \\
\hline \multicolumn{6}{|l|}{ Fabaceae } \\
\hline Pentaclethra macroloba ${ }^{\#}$ & Bark & C. atrox & - & Hemorrhage* & {$[145]$} \\
\hline Schizolobium parahyba & Leaf & C.d. terrificus & $\mathrm{PLA}_{2}$ & Edema* & {$[146,147]$} \\
\hline \multicolumn{6}{|l|}{ Musaceae } \\
\hline Musa $\times$ paradisiaca $^{\#}$ & Exudate & C. d. terrificus & $\mathrm{PLA}_{2}$ & - & {$[155]$} \\
\hline \multicolumn{6}{|l|}{ Rubiaceae } \\
\hline Randia aculeata & Fruit & C. simus & - & Myotoxicity & {$[78]$} \\
\hline \multicolumn{6}{|l|}{ Sapindaceae } \\
\hline Sapindus saponaria & $\begin{array}{l}\text { In vitro cultivated } \\
\text { callus }\end{array}$ & C.d. terrificus & $\mathrm{PLA}_{2}$ & - & {$[161]$} \\
\hline
\end{tabular}

its poor efficacy against local effects, the treatment with medicinal plants may provide a potential adjuvant alternative to treat snakebites, being used to complement the activity and effectiveness of available snake venom therapy. The main potential advantages of antiophidic plants are their low cost, easy access, stability at room temperature, and ability to neutralize a broad spectrum of toxins, including the local tissue damage.

Interestingly, some studies have showed that the crude extracts are more powerful than the individual herbal compounds, which could, at a certain extent, justify the development of herbal products containing these plants instead of medicines containing isolated compounds, which in turn could be more rapidly available in market, after proof of safety, effectiveness, and quality of these products. However, despite the existence of many plants with great potential, no natural antiophidic product is available in market, which points to question of the need for further studies. Only a few numbers of patents regarding herbal products against snakebites were found in literature. Some patents regarding the use of Chinese medicinal plants against snake and bug bites were found. In our research group, two patents were deposited concerning the processes of obtaining extracts, fraction, isolated compounds, and pharmaceutical compositions of some plants studied by our group applied in the treatment of accidents with venomous animals (BR 102013 0340464 A2 and BR 1020120269589 A2). Thus, the number of patents with antiophidic herbal products is still relatively small. For this reason, we encourage pharmacologists and toxinologists around the world to intensify studies with antiophidic plants, especially prioritizing those with the greatest number of indications in traditional medicine and emphasizing clinical studies with the most active plants in preclinical studies, given that the low number of human studies is one of the major obstacles for the future application of herbal products with antiophidic potential. No less important, toxicological studies are also extremely necessary to ensure the safety of these products.

In conclusion, the data presented in this review provides an updated scenario for and insights into future research aiming at validation of medicinal plants as antiophidic agents and, based on scientific evidences, strengthens the potentiality of medicinal plants and ethnopharmacological knowledge as a tool for design of potent inhibitors and/or herbal medicines against venom toxins.

\section{Conflicts of Interest}

The authors declare that there are no conflicts of interest regarding the publication of this paper.

\section{Acknowledgments}

Matheus de Freitas Fernandes-Pedrosa is CNPq fellowshiphonored researcher. Juliana Félix-Silva acknowledges CAPES for the Ph.D. scholarship. 
TABLE 8: List of medicinal plants with inhibitory potential against local effects induced by other snakes.

\begin{tabular}{|c|c|c|c|c|c|}
\hline \multirow{2}{*}{ Plant name } & \multirow{2}{*}{ Part used } & \multirow{2}{*}{ Snake venom } & \multicolumn{2}{|c|}{ Inhibited activities } & \multirow{2}{*}{ Reference(s) } \\
\hline & & & In vitro & In vivo & \\
\hline \multicolumn{6}{|l|}{ Amaryllidaceae } \\
\hline Crinum jagus & Bulb & Echis ocellatus & Hemorrhage & Myotoxicity $^{* *}$ & {$[167,191]$} \\
\hline \multicolumn{6}{|l|}{ Asteraceae } \\
\hline Artemisia absinthium & Aerial parts & $\begin{array}{l}\text { Montivipera } \\
\text { xanthina }\end{array}$ & - & Edema & [192] \\
\hline Mikania laevigata & Leaf & Philodryas olfersii & $\begin{array}{l}\text { Inflammation, } \\
\text { myotoxicity }\end{array}$ & - & {$[193]$} \\
\hline \multicolumn{6}{|l|}{ Fabaceae } \\
\hline Albizia lebbeck ${ }^{\#}$ & Seed & Echis carinatus & Proteolytic, SVH & $\begin{array}{l}\text { Hemorrhage, } \\
\text { myotoxicity }\end{array}$ & {$[194]$} \\
\hline Mimosa pudica ${ }^{\#}$ & Root & $\begin{array}{l}\text { Bungarus caeruleus, } \\
\text { Echis carinatus }\end{array}$ & $\mathrm{PLA}_{2}$, proteolytic, SVH & Edema* & {$[91,92]$} \\
\hline Parkia biglobosa & Stem bark & Echis ocellatus & $\begin{array}{c}\text { Cytotoxicity against } \\
\text { muscle cells, } \\
\text { hemorrhage }\end{array}$ & - & {$[93]$} \\
\hline Pentaclethra macroloba & Bark & $\begin{array}{l}\text { Calloselasma } \\
\text { rhodostoma }\end{array}$ & - & Hemorrhage $^{*}$ & {$[145]$} \\
\hline $\begin{array}{l}\text { Senna auriculata (syn. Cassia } \\
\text { auriculata) }\end{array}$ & Leaf & Echis carinatus & $\mathrm{PLA}_{2}$, proteolytic, $\mathrm{SVH}$ & $\begin{array}{l}\text { Edema }^{* * *}, \\
\text { hemorrhage }^{* * *}, \\
\text { myotoxicity }^{* * *}\end{array}$ & {$[195]$} \\
\hline \multicolumn{6}{|l|}{ Malvaceae } \\
\hline Hibiscus aethiopicus & Whole plant & $\begin{array}{l}\text { Echis carinatus, } \\
\text { Echis ocellatus }\end{array}$ & $\begin{array}{l}\text { Cytotoxicity against } \\
\text { muscle cells, } \\
\text { hemorrhage }\end{array}$ & $\begin{array}{l}\text { Edema }^{* * *} \\
\text { hemorrhage }^{* * *}\end{array}$ & {$[196,197]$} \\
\hline \multicolumn{6}{|l|}{ Salvadoraceae } \\
\hline $\begin{array}{l}\text { Azima tetracantha } \\
\text { Vitaceae }\end{array}$ & Leaf & Bungarus caeruleus & $\mathrm{PLA}_{2}$ & - & {$[181]$} \\
\hline Vitis vinifera & Seed & Echis carinatus & Proteolytic, SVH & $\begin{array}{c}\text { Edema* }{ }^{*} \text { hemorrhage }{ }^{*}, \\
\text { myotoxicity* }\end{array}$ & {$[198]$} \\
\hline
\end{tabular}

$\mathrm{PLA}_{2}=$ snake venom phospholipase $\mathrm{A}_{2} ; \mathrm{SVH}=$ snake venom hyaluronidase. ${ }^{\#}$ Vegetal species with related folk use as antiophidic agents, as showed in Table 1 . ${ }^{*}$ Studies where inhibitory activity was assessed only by preincubation of venom with extract (see Section 4.1 for details). ${ }^{* *}$ Active in preincubation tests but inactive or only poorly active when extract was used independently of venom (pre-, co-, or posttreatment protocols). ${ }^{* * *}$ Active in preincubation tests and when used independently of venom (pre-, co-, or posttreatment protocols).

\section{References}

[1] J. M. Gutiérrez, T. Burnouf, R. A. Harrison et al., "A call for incorporating social research in the global struggle against snakebite," PLoS Neglected Tropical Diseases, vol. 9, no. 9, article e0003960, 2015.

[2] A. Kasturiratne, A. R. Wickremasinghe, N. de Silva et al., "The global burden of snakebite: a literature analysis and modelling based on regional estimates of envenoming and deaths," PLoS Medicine, vol. 5, no. 11, article e218, 2008.

[3] J. M. Gutiérrez, D. A. Warrell, D. J. Williams et al., “The need for full integration of snakebite envenoming within a global strategy to combat the neglected tropical diseases: the way forward," PLoS Neglected Tropical Diseases, vol. 7, no. 6, Article ID e2162, 2013.

[4] R. A. Harrison, A. Hargreaves, S. C. Wagstaff, B. Faragher, and D. G. Lalloo, "Snake envenoming: a disease of poverty," PLoS Neglected Tropical Diseases, vol. 3, no. 12, article e569, 2009.

[5] D. A. Warrell, "Venomous animals," Medicine, vol. 40, no. 3, pp. 159-163, 2012.
[6] C. L. S. Guimarães, L. S. Moreira-Dill, R. S. Fernandes et al., "Biodiversity as a source of bioactive compounds against snakebites," Current Medicinal Chemistry, vol. 21, no. 25, pp. 29522979, 2014.

[7] T. S. Kang, D. Georgieva, N. Genov et al., "Enzymatic toxins from snake venom: structural characterization and mechanism of catalysis," The FEBS Journal, vol. 278, no. 23, pp. 4544-4576, 2011.

[8] J. M. Guti@rrez and B. Lomonte, "Local tissue damage induced by Bothrops snake venoms. A review," Memorias do Instituto Butantan, vol. 51, no. 4, pp. 211-23, 1989.

[9] J. M. Gutiérrez, G. León, and T. Burnouf, "Antivenoms for the treatment of snakebite envenomings: the road ahead," Biologicals, vol. 39, no. 3, pp. 129-142, 2011.

[10] G. León, M. Herrera, Á. Segura, M. Villalta, M. Vargas, and J. M. Gutiérrez, "Pathogenic mechanisms underlying adverse reactions induced by intravenous administration of snake antivenoms," Toxicon, vol. 76, pp. 63-76, 2013.

[11] W. B. Mors, M. C. do Nascimento, J. P. Parente, M. H. da Silva, P. A. Melo, and G. Suarez-Kurtz, "Neutralization of lethal 
and myotoxic activities of south american rattlesnake venom by extracts and constituents of the plant Eclipta prostrata (Asteraceae)," Toxicon, vol. 27, no. 9, pp. 1003-1009, 1989.

[12] M. S. Santhosh, M. Hemshekhar, K. Sunitha et al., "Snake venom induced local toxicities: plant secondary metabolites as an auxiliary therapy," Mini-Reviews in Medicinal Chemistry, vol. 13, no. 1, pp. 106-123, 2013.

[13] A. Shabbir, M. Shahzad, P. Masci, and G. C. Gobe, "Protective activity of medicinal plants and their isolated compounds against the toxic effects from the venom of Naja (cobra) species," Journal of Ethnopharmacology, vol. 157, pp. 222-227, 2014.

[14] A. Dey and J. N. de, "Phytopharmacology of antiophidian botanicals: A review," International Journal of Pharmacology, vol. 8, no. 2, pp. 62-79, 2012.

[15] A. Gomes, R. Das, S. Sarkhel et al., "Herbs and herbal constituents active against snake bite," Indian Journal of Experimental Biology, vol. 48, no. 9, pp. 865-878, 2010.

[16] A. Sulochana, D. Raveendran, A. Krishnamma, and O. Oommen, "Ethnomedicinal plants used for snake envenomation by folk traditional practitioners from Kallar forest region of South Western Ghats, Kerala, India," Journal of Intercultural Ethnopharmacology, vol. 4, no. 1, p. 47, 2015.

[17] M. A. Butt, M. Ahmad, A. Fatima et al., "Ethnomedicinal uses of plants for the treatment of snake and scorpion bite in Northern Pakistan," Journal of Ethnopharmacology, vol. 168, pp. 164-181, 2015.

[18] R. M. Dharmadasa, G. C. Akalanka, P. R. M. Muthukumarana, and R. G. S. Wijesekara, "Ethnopharmacological survey on medicinal plants used in snakebite treatments in Western and Sabaragamuwa provinces in Sri Lanka," Journal of Ethnopharmacology, vol. 179, pp. 110-127, 2016.

[19] C. P. Kala, "Herbal treatment for snakebites in Uttarakhand state of India," Indian Journal of Natural Products and Resources, vol. 6, no. 1, pp. 56-61, 2015.

[20] R. P. Samy, M. M. Thwin, P. Gopalakrishnakone, and S. Ignacimuthu, "Ethnobotanical survey of folk plants for the treatment of snakebites in Southern part of Tamilnadu, India," Journal of Ethnopharmacology, vol. 115, no. 2, pp. 302-312, 2008.

[21] M. Rajadurai, V. G. Vidhya, M. Ramya, and A. Bhaskar, "Ethnomedicinal plants used by the traditional healers of pachamalai hills, Tamilnadu, India," Studies on Ethno-Medicine, vol. 3, no. 1, pp. 39-41, 2009.

[22] S. Sarkhel, "Ethnobotanical survey of folklore plants used in treatment of snakebite in Paschim Medinipur district, West Bengal," Asian Pacific Journal of Tropical Biomedicine, vol. 4, no. 5, pp. 416-420, 2014.

[23] G. Penchalapratap, G. Sudarsanam, R. Pushpan, and G. P. Prasad, "Herbal remedies for snake bites in ethnic practices of Chittoor District, Andhra Pradesh," Ancient Science of Life, vol. 29, no. 4, pp. 13-16, 2010.

[24] A. K. Singh, A. S. Raghubanshi, and J. S. Singh, "Medical ethnobotany of the tribals of Sonaghati of Sonbhadra district, Uttar Pradesh, India," Journal of Ethnopharmacology, vol. 81, no. 1, pp. 31-41, 2002.

[25] C. Kadel and A. K. Jain, "Folklore claims on snakebite among some tribal communities of Central India," Indian Journal of Traditional Knowledge, vol. 7, no. 2, pp. 296-299, 2008.

[26] K. Yesodharan and K. A. Sujana, "Ethnomedicinal knowledge among Malamalasar tribe of Parambikulam wildlife sanctuary," Indian Journal of Traditional Knowledge, vol. 6, no. 3, pp. 481-85, 2007.
[27] F. G. Coe and G. J. Anderson, "Snakebite ethnopharmacopoeia of eastern Nicaragua," Journal of Ethnopharmacology, vol. 96, no. 1-2, pp. 303-323, 2005.

[28] M. Prabu and R. Kumuthakalavalli, "Folk remedies of medicinal plants for snake bites, scorpion stings and dog bites in Eastern Ghats of Kolli Hills, Tamil Nadu, India," International Journal of Research in Ayurveda and Pharmacy, vol. 3, no. 5, pp. 696-700, 2012.

[29] J. Sanz-Biset, J. Campos-de-la-Cruz, M. A. Epiquién-Rivera, and S. Cañigueral, "A first survey on the medicinal plants of the Chazuta valley (Peruvian Amazon)," Journal of Ethnopharmacology, vol. 122, no. 2, pp. 333-362, 2009.

[30] M. N. Hasan, M. N. K. Azam, M. N. Ahmed, and A. Hirashima, "A randomized ethnomedicinal survey of snakebite treatment in southwestern parts of Bangladesh," Journal of Traditional and Complementary Medicine, vol. 6, no. 4, pp. 337-342, 2015.

[31] B. O. Owuor and D. P. Kisangau, "Kenyan medicinal plants used as antivenin: a comparison of plant usage," Journal of Ethnobiology and Ethnomedicine, vol. 2, no. 1, article 7, 2006.

[32] M. F. Kadir, J. R. Karmoker, M. R. Alam, S. R. Jahan, S. Mahbub, and M. M. K. Mia, "Ethnopharmacological survey of medicinal plants used by traditional healers and indigenous people in Chittagong Hill Tracts, Bangladesh, for the treatment of snakebite," Evidence-Based Complementary and Alternative Medicine, vol. 2015, Article ID 871675, 23 pages, 2015.

[33] V. M. De Moura, L. A. Freitas De Sousa, M. Cristina Dos-Santos et al., "Plants used to treat snakebites in Santarém, western Pará, Brazil: an assessment of their effectiveness in inhibiting hemorrhagic activity induced by Bothrops jararaca venom," Journal of Ethnopharmacology, vol. 161, pp. 224-232, 2015.

[34] J. Vásquez, J. C. Alarcón, S. L. Jiménez et al., "Main plants used in traditional medicine for the treatment of snake bites $n$ the regions of the department of Antioquia, Colombia," Journal of Ethnopharmacology, vol. 170, pp. 158-166, 2015.

[35] R. Otero, R. Fonnegra, S. L. Jiménez et al., "Snakebites and ethnobotany in the northwest region of Colombia. Part I: traditional use of plants," Journal of Ethnopharmacology, vol. 71, no. 3, pp. 493-504, 2000.

[36] M. Rahmatullah, A. Hasan, W. Parvin et al., "Medicinal plants and formulations used by the Soren clan of the Santal tribe in Rajshahi District, Bangladesh for treatment of various ailments," African Journal of Traditional, Complementary and Alternative Medicines, vol. 9, no. 3, pp. 350-359, 2012.

[37] M. Rigat, J. Vallès, U. Dambrosio, A. Gras, J. Iglésias, and T. Garnatje, "Plants with topical uses in the Ripollès district (Pyrenees, Catalonia, Iberian Peninsula): Ethnobotanical survey and pharmacological validation in the literature," Journal of Ethnopharmacology, vol. 164, pp. 162-179, 2015.

[38] A. Jain, S. S. Katewa, S. K. Sharma, P. Galav, and V. Jain, "Snakelore and indigenous snakebite remedies practiced by some tribals of Rajasthan," Indian Journal of Traditional Knowledge, vol. 10, no. 2, pp. 258-268, 2011.

[39] A. N. Shukla, S. Srinivastan, and A. K. S. Rawat, "An ethnobotanical study of medicinal plants of Rewa district, Madhya Pradesh," Indian Journal of Traditional Knowledge, vol. 9, no. 1, pp. 191-202, 2010.

[40] M. Panghal, V. Arya, S. Yadav, S. Kumar, and J. P. Yadav, "Indigenous knowledge of medicinal plants used by Saperas community of Khetawas, Jhajjar District, Haryana, India," Journal of Ethnobiology and Ethnomedicine, vol. 6, article 4, 2010.

[41] A. V. Khan, Q. U. Ahmed, M. W. Khan, and A. A. Khan, "Herbal cure for poisons and poisonous bites from Western 
Uttar Pradesh, India," Asian Pacific Journal of Tropical Disease, vol. 4, no. 1, pp. S116-S120, 2014.

[42] M. Sikdar and U. Dutta, "Traditional phytotherapy among the Nath people of Assam," Studies on Ethno-Medicine, vol. 2, no. 1, pp. 39-45, 2008.

[43] M. A. Khan, S. A. Khan, M. A. Quresh et al., "Ethnobotany of some useful plants of poonch valley azad kashmir," Journal of Medicinal Plant Research, vol. 5, no. 26, pp. 6140-6151, 2011.

[44] J. Vásquez, S. L. Jiménez, I. C. Gómez et al., "Snakebites and ethnobotany in the eastern region of Antioquia, Colombia-the traditional use of plants," Journal of Ethnopharmacology, vol. 146, no. 2, pp. 449-455, 2013.

[45] B. Sivasankari, M. Anandharaj, and P. Gunasekaran, "An ethnobotanical study of indigenous knowledge on medicinal plants used by the village peoples of Thoppampatti, Dindigul district, Tamilnadu, India," Journal of Ethnopharmacology, vol. 153, no. 2, pp. 408-423, 2014.

[46] D. Mebs, "Notes on the traditional use of plants to treat snake bite in northern Papua New Guinea," Toxicon, vol. 38, no. 2, pp. 299-302, 2000.

[47] M. D. F. Agra, K. N. Silva, I. J. L. D. Basílio, P. F. De Freitas, and J. M. Barbosa-Filho, "Survey of medicinal plants used in the region Northeast of Brazil," Brazilian Journal of Pharmacognosy, vol. 18, no. 3, pp. 472-508, 2008.

[48] R. A. Ritter, M. V. B. Monteiro, F. O. B. Monteiro et al., "Ethnoveterinary knowledge and practices at Colares island, Pará state, eastern Amazon, Brazil," Journal of Ethnopharmacology, vol. 144, no. 2, pp. 346-352, 2012.

[49] K. S. Murthy, P. C. Sharma, and P. Kishore, “Tribal remedies for snakebite from Orissa," Ancient Science of Life, vol. 6, no. 2, pp. 122-123, 1986.

[50] L. C. Di Stasi and C. A. Hiruma-Lima, Plantas medicinais na Amazônia e na Mata Atlântica, UNESP, São Paulo, Brazil, 2nd edition, 2002.

[51] A. Belayneh and N. F. Bussa, "Ethnomedicinal plants used to treat human ailments in the prehistoric place of Harla and Dengego valleys, eastern Ethiopia," Journal of Ethnobiology and Ethnomedicine, vol. 10, article 18, 2014.

[52] M. Ayyanar and S. Ignacimuthu, "Ethnobotanical survey of medicinal plants commonly used by Kani tribals in Tirunelveli hills of Western Ghats, India," Journal of Ethnopharmacology, vol. 134, no. 3, pp. 851-864, 2011.

[53] F. Haq, H. Ahmad, and M. Alam, "Traditional uses of medicinal plants of Nandiar Khuwarr catchment (District Battagram), Pakistan," Journal of Medicinal Plants Research, vol. 5, no. 1, pp. 39-48, 2011.

[54] A. Cheikhyoussef, M. Shapi, K. Matengu, and H. M. Ashekele, "Ethnobotanical study of indigenous knowledge on medicinal plant use by traditional healers in Oshikoto region, Namibia," Journal of Ethnobiology and Ethnomedicine, vol. 7, article 10, 2011.

[55] A. Jain, S. S. Katewa, P. K. Galav, and P. Sharma, "Medicinal plant diversity of Sitamata wildlife sanctuary, Rajasthan, India," Journal of Ethnopharmacology, vol. 102, no. 2, pp. 143-157, 2005.

[56] H. C. Dutt, N. Bhagat, and S. Pandita, "Oral traditional knowledge on medicinal plants in jeopardy among Gaddi shepherds in hills of northwestern Himalaya, J\&K, India," Journal of Ethnopharmacology, vol. 168, pp. 337-348, 2015.

[57] G. Odonne, C. Valadeau, J. Alban-Castillo, D. Stien, M. Sauvain, and G. Bourdy, "Medical ethnobotany of the Chayahuita of the Paranapura basin (Peruvian Amazon)," Journal of Ethnopharmacology, vol. 146, no. 1, pp. 127-153, 2013.
[58] M. Jankovic, "Communication and shared information," Philosophical Studies. An International Journal for Philosophy in the Analytic Tradition, vol. 169, no. 3, pp. 489-508, 2014.

[59] M. R. Hernandez, C. H. Avila-Bello, and J. E. Morales-Mavil, "Etnobotánica y ecología de plantas utilizadas por tres curanderos contra la mordedura de serpiente en la región de Acayucan, Veracruz, México," Boletin de la Sociedad Botanica de Mexico, vol. 81, pp. 89-100, 2007.

[60] M. Rahmatullah, D. Ferdausi, M. A. H. Mollik, R. Jahan, M. H. Chowdhury, and W. M. Haque, "A survey of medicinal plants used by Kavirajes of Chalna area, Khulna district, Bangladesh," African Journal of Traditional, Complementary and Alternative Medicines, vol. 7, no. 2, pp. 91-97, 2010.

[61] P. Giovannini, "Medicinal plants of the Achuar (Jivaro) of Amazonian Ecuador: ethnobotanical survey and comparison with other Amazonian pharmacopoeias," Journal of Ethnopharmacology, vol. 164, pp. 78-88, 2015.

[62] P. Karuppaiya and H. S. Tsay, "Therapeutic values, chemical constituents and toxicity of Taiwanese Dysosma pleiantha: a review," Toxicology Letters, vol. 236, no. 2, pp. 90-97, 2015.

[63] R. D. Kshirsagar and N. P. Singh, "Some less known ethnomedicinal uses from Mysore and Coorg districts, Karnataka state, India," Journal of Ethnopharmacology, vol. 75, no. 2-3, pp. 231238, 2001.

[64] U. P. De Albuquerque and L. D. H. C. Andrade, "Uso de recursos vegetais da caatinga: $\mathrm{O}$ caso do agreste do Estado de Pernambuco (Nordeste do Brasil)," Interciencia, vol. 27, no. 7, pp. 336-346, 2002.

[65] A. Cheikhyoussef and W. Embashu, "Ethnobotanical knowledge on indigenous fruits in Ohangwena and Oshikoto regions in Northern Namibia," Journal of Ethnobiology and Ethnomedicine, vol. 9, no. 1, article 34, 2013.

[66] A. Shah, K. A. Bharati, J. Ahmad, and M. Sharma, "New ethnomedicinal claims from Gujjar and Bakerwals tribes of Rajouri and Poonch districts of Jammu and Kashmir, India," Journal of Ethnopharmacology, vol. 166, pp. 119-128, 2015.

[67] H. C. Ong and M. Nordiana, "Malay ethno-medico botany in Machang, Kelantan, Malaysia," Fitoterapia, vol. 70, no. 5, pp. 502-513, 1999.

[68] R. Seebaluck, A. Gurib-Fakim, and F. Mahomoodally, "Medicinal plants from the genus Acalypha (Euphorbiaceae): a review of their ethnopharmacology and phytochemistry," Journal of Ethnopharmacology, vol. 159, pp. 137-157, 2015.

[69] P. Sharma and U. Devi, "Ethnobotanical uses of biofencing plants in Himachal Pradesh, Northwest Himalaya," Pakistan Journal of Biological Sciences, vol. 16, no. 24, pp. 1957-1963, 2013.

[70] M. Coelho-Ferreira, "Medicinal knowledge and plant utilization in an Amazonian coastal community of Marudá, Pará State (Brazil)," Journal of Ethnopharmacology, vol. 126, no. 1, pp. 159175, 2009.

[71] F. C. S. Oliveira, R. F. M. Barros, and J. M. Moita Neto, "Medicinal plants used in rural communities from Oeiras Municipality, in the semi-arid region of Piauí State (PI), Brazil," Revista Brasileira de Plantas Medicinais, vol. 12, no. 3, pp. 282-301, 2010.

[72] C. G. Crepaldi, J. L. A. Campos, U. P. Albuquerque, and M. F. Sales, "Richness and ethnobotany of the family Euphorbiaceae in a tropical semiarid landscape of Northeastern Brazil," South African Journal of Botany, vol. 102, pp. 157-165, 2016.

[73] M. Murugesan, V. Balasubramaniam, and H. Arthi, "Ethno medical knowledge of plants used by Irula Tribes, Chengal Combai, the Nilgiris, Tamilnadu," Ancient Science of Life, vol. 24, no. 4, pp. 179-82, 2005. 
[74] B. N. Nsuala, G. Enslin, and A. Viljoen, "“Wild cannabis": a review of the traditional use and phytochemistry of Leonotis leonurus," Journal of Ethnopharmacology, vol. 174, pp. 520-539, 2015.

[75] M. K. Swamy and U. R. Sinniah, "A Comprehensive review on the phytochemical constituents and pharmacological activities of Pogostemon cablin Benth.: an Aromatic Medicinal Plant of Industrial Importance," Molecules, vol. 20, no. 5, pp. 8521-8547, 2015.

[76] D. W. Gakuya, S. M. Itonga, J. M. Mbaria, J. K. Muthee, and J. K. Musau, "Ethnobotanical survey of biopesticides and other medicinal plants traditionally used in Meru central district of Kenya," Journal of Ethnopharmacology, vol. 145, no. 2, pp. 547553, 2013.

[77] T. K. Hazarika, Lalramchuana, and B. P. Nautiyal, "Studies on wild edible fruits of Mizoram, India used as ethno-medicine," Genetic Resources and Crop Evolution, vol. 59, no. 8, pp. 17671776, 2012.

[78] C. A. Gallardo-Casas, G. Guevara-Balcázar, E. Morales-Ramos et al., "Ethnobotanic study of Randia aculeata (Rubiaceae) in Jamapa, Veracruz, Mexico, and its anti-snake venom effects on mouse tissue," Journal of Venomous Animals and Toxins Including Tropical Diseases, vol. 18, no. 3, pp. 287-294, 2012.

[79] L. Xia, Q. Guo, P. Tu, and X. Chai, "The genus Casearia: a phytochemical and pharmacological overview," Phytochemistry Reviews, pp. 1-37, 2014.

[80] M. Molander, C. H. Saslis-Lagoudakis, A. K. Jäger, and N. Rønsted, "Cross-cultural comparison of medicinal floras used against snakebites," Journal of Ethnopharmacology, vol. 139, no. 3, pp. 863-872, 2012.

[81] J. Félix-Silva, R. B. Giordani, A. A. D. Silva-Jr, S. M. Zucolotto, and M. D. F. Fernandes-Pedrosa, "Jatropha gossypiifolia L. (Euphorbiaceae): a review of traditional uses, phytochemistry, pharmacology, and toxicology of this medicinal plant," Evidence-Based Complementary and Alternative Medicine, vol. 2014, Article ID 369204, 32 pages, 2014.

[82] M. Molander, L. Nielsen, S. Søgaard et al., "Hyaluronidase, phospholipase $\mathrm{A}_{2}$ and protease inhibitory activity of plants used in traditional treatment of snakebite-induced tissue necrosis in Mali, DR Congo and South Africa," Journal of Ethnopharmacology, vol. 157, pp. 171-180, 2014.

[83] N. Thangavel and J. K. Gupta, "Anti-inflammatory and antisnake venom activity of Andrographis stenophylla leaf," Asian Journal of Chemistry, vol. 19, no. 2, pp. 1307-1312, 2007.

[84] M. H. H. B. Asad, M. T. Razi, Durr-e-Sabih et al., "Anti-venom potential of Pakistani medicinal plants: inhibition of anticoagulation activity of Naja naja karachiensis toxin," Current Science, vol. 105, no. 10, pp. 1419-1424, 2013.

[85] G. Sakthivel, A. Dey, K. Nongalleima et al., "In vitro and in vivo evaluation of polyherbal formulation against Russell's viper and cobra venom and screening of bioactive components by docking studies," Evidence-Based Complementary and Alternative Medicine, vol. 2013, Article ID 781216, 12 pages, 2013.

[86] P. Soni and S. H. Bodakhe, "Antivenom potential of ethanolic extract of Cordia macleodii bark against Naja venom," Asian Pacific Journal of Tropical Biomedicine, vol. 4, pp. S449-S454, 2014.

[87] M. A. Ibrahim, A. B. Aliyu, A. Abusufiyanu, M. Bashir, and A. B. Sallau, "Inhibition of naja nigricolis (Reinhardt) venom protease activity by Luffa egyptiaca (Mill) and Nicotiana rustica (Linn) extracts," Indian Journal of Experimental Biology, vol. 49, no. 7, pp. 552-554, 2011.
[88] K. Gopi, K. Renu, B. Sannanaik Vishwanath, and G. Jayaraman, "Protective effect of Euphorbia hirta and its components against snake venom induced lethality," Journal of Ethnopharmacology, vol. 165, pp. 180-190, 2015.

[89] K. V. N. Rathnakar Reddi, S. S. Rajesh, K. Narendra et al., "In vitro anti-venom potential of various Jatropha extracts on neutralizing cytotoxic effect induced by phospholipase A2 of crude venom from Indian cobra (Naja naja)," Bangladesh Journal of Pharmacology, vol. 9, no. 1, pp. 22-28, 2014.

[90] M. Mahanta and A. K. Mukherjee, "Neutralisation of lethality, myotoxicity and toxic enzymes of Naja kaouthia venom by Mimosa pudica root extracts," Journal of Ethnopharmacology, vol. 75, no. 1, pp. 55-60, 2001.

[91] K. S. Girish, H. P. Mohanakumari, S. Nagaraju, B. S. Vishwanath, and K. Kemparaju, "Hyaluronidase and protease activities from Indian snake venoms: Neutralization by Mimosa pudica root extract," Fitoterapia, vol. 75, no. 3-4, pp. 378-380, 2004.

[92] S. Meenatchisundaram, S. Priyagrace, R. Vijayaraghavan, A. Velmurugan, G. Parameswari, and A. Michael, "Antitoxin activity of Mimosa pudica root extracts against Naja naja and Bangarus caerulus venoms," Bangladesh Journal of Pharmacology, vol. 4, no. 2, pp. 105-109, 2009.

[93] I. U. Asuzu and A. L. Harvey, "The antisnake venom activities of Parkia biglobosa (Mimosaceae) stem bark extract," Toxicon, vol. 42, no. 7, pp. 763-768, 2003.

[94] K. Gopi, K. Renu, and G. Jayaraman, "Inhibition of Naja naja venom enzymes by the methanolic extract of Leucas aspera and its chemical profile by GC-MS," Toxicology Reports, vol. 1, pp. 667-673, 2014.

[95] I. Chatterjee, A. K. Chakravarty, and A. Gomes, "Antisnake venom activity of ethanolic seed extract of Strychnos nux vomica Linn," Indian Journal of Experimental Biology, vol. 42, no. 5, pp. 468-475, 2004.

[96] J. Félix-Silva, T. Souza, Y. A. S. Menezes et al., "Aqueous leaf extract of Jatropha gossypiifolia L. (Euphorbiaceae) inhibits enzymatic and biological actions of Bothrops jararaca snake venom," PLoS ONE, vol. 9, no. 8, Article ID e104952, 2014.

[97] WHO, Guidelines for the Production, Control and Regulation of Snake Antivenom Immunoglobulins, WHO Press, Geneva, Switzerland, 2010.

[98] V. Mourão De Moura, A. N. Serra Bezerra, R. H. Veras Mourão et al., "A comparison of the ability of Bellucia dichotoma Cogn. (Melastomataceae) extract to inhibit the local effects of Bothrops atrox venom when pre-incubated and when used according to traditional methods," Toxicon, vol. 85, pp. 59-68, 2014.

[99] F. F. A. Fernandes, M. A. Tomaz, C. Z. El-Kik et al., "Counteraction of Bothrops snake venoms by Combretum leprosum root extract and arjunolic acid," Journal of Ethnopharmacology, vol. 155, no. 1, pp. 552-562, 2014.

[100] E. C. De Oliveira, C. P. Fernandes, E. F. Sanchez, L. Rocha, and A. L. Fuly, "Inhibitory effect of plant Manilkara subsericea against biological activities of Lachesis muta snake venom," BioMed Research International, vol. 2014, Article ID 408068, 2014.

[101] J. Saturnino-Oliveira, D. D. C. Santos, A. G. Guimarães et al., "Abarema cochliacarpos extract decreases the inflammatory process and skeletal muscle injury induced by bothrops leucurus venom," BioMed Research International, vol. 2014, Article ID 820761, 9 pages, 2014. 
[102] M. A. Strauch, M. A. Tomaz, M. Monteiro-Machado et al., "Antiophidic activity of the extract of the Amazon plant Humirianthera ampla and constituents," Journal of Ethnopharmacology, vol. 145, no. 1, pp. 50-58, 2013.

[103] P. J. Houghton and I. M. Osibogun, "Flowering plants used against snakebite," Journal of Ethnopharmacology, vol. 39, no. 1, pp. 1-29, 1993.

[104] P. A. Shenoy, S. S. Nipate, J. M. Sonpetkar, N. C. Salvi, A. B. Waghmare, and P. D. Chaudhari, "Anti-snake venom activities of ethanolic extract of fruits of Piper longum L. (Piperaceae) against Russell's viper venom: characterization of piperine as active principle," Journal of Ethnopharmacology, vol. 147, no. 2, pp. 373-382, 2013.

[105] S. D. Araújo, A. de Souza, F. P. B. Nunes, and L. R. C. Gonçalves, "Effect of dexamethasone associated with serum therapy on treatment of Bothrops jararaca venom-induced paw edema in mice," Inflammation Research, vol. 56, no. 10, pp. 409-413, 2007.

[106] F. C. Patrão-Neto, M. A. Tomaz, M. A. Strauch et al., "Dexamethasone antagonizes the in vivo myotoxic and inflammatory effects of Bothrops venoms," Toxicon, vol. 69, pp. 55-64, 2013.

[107] I. Gómez-Betancur, D. Benjumea, A. Patiño, N. Jiménez, and E. Osorio, "Inhibition of the toxic effects of Bothrops asper venom by pinostrobin, a flavanone isolated from Renealmia alpinia (Rottb.) MAAS," Journal of Ethnopharmacology, vol. 155, no. 3, pp. 1609-1615, 2014.

[108] A. Magalhães, G. B. D. Santos, M. C. D. S. Verdam et al., "Inhibition of the inflammatory and coagulant action of Bothrops atrox venom by the plant species Marsypianthes chamaedrys," Journal of Ethnopharmacology, vol. 134, no. 1, pp. 82-88, 2011.

[109] I. C. Pereira, A. M. Barbosa, M. J. Salvador et al., "Anti-inflammatory activity of Blutaparon portulacoides ethanolic extract against the inflammatory reaction induced by Bothrops jararacussu venom and isolated myotoxins BthTX-I and II," Journal of Venomous Animals and Toxins Including Tropical Diseases, vol. 15, no. 3, pp. 527-545, 2009.

[110] F. P. Reis, I. M. Senna Bonfa, R. B. Cavalcante et al., “Tabebuia aurea decreases inflammatory, myotoxic and hemorrhagic activities induced by the venom of Bothrops neuwiedi," Journal of Ethnopharmacology, pp. 352-357, 2014.

[111] J. A. Pereañez, T. Lobo-Echeverri, B. Rojano et al., "Correlation of the inhibitory activity of phospholipase A2 snake venom and the antioxidant activity of Colombian plant extracts," Brazilian Journal of Pharmacognosy, vol. 20, no. 6, pp. 910-916, 2010.

[112] K. Sunitha, M. Hemshekhar, R. M. Thushara et al., "Inflammation and oxidative stress in viper bite: an insight within and beyond," Toxicon, vol. 98, pp. 89-97, 2015.

[113] P. Saravia-Otten, J. M. Gutiérrez, S. Arvidson, M. Thelestam, and J.-I. Flock, "Increased infectivity of Staphylococcus aureus in an experimental model of snake venom-induced tissue damage," Journal of Infectious Diseases, vol. 196, no. 5, pp. 748-754, 2007.

[114] M. T. Jorge and L. A. Ribeiro, "Infections in the bite site after envenoming by snakes of the Bothrops genus," Journal of Venomous Animals and Toxins, vol. 3, no. 2, pp. 264-272, 1997.

[115] T. P. D. Silva, V. M. D. Moura, M. C. S. D. Souza et al., "Connarus favosus Planch.: An inhibitor of the hemorrhagic activity of Bothrops atrox venom and a potential antioxidant and antibacterial agent," Journal of Ethnopharmacology, vol. 183, pp. 166-175, 2016.

[116] R. Dehghani, M. R. Sharif, R. Moniri, A. Sharif, and H. H. Kashani, "The identification of bacterial flora in oral cavity of snakes," Comparative Clinical Pathology, vol. 25, no. 2, pp. 279283, 2016.

[117] P. Hearn, T. Miliya, S. Hor, V. Sar, and P. Turner, "Necrotizing fasciitis complicating snakebite in Cambodia," IDCases, vol. 2, no. 3, article 70, pp. 86-87, 2015.

[118] D. S. Palappallil, "Pattern of use of antibiotics following snake bite in a tertiary care hospital," Journal of Clinical and Diagnostic Research, vol. 9, no. 8, pp. OC05-OC09, 2015.

[119] J. Félix-Silva, J. A. Gomes, J. B. Xavier-Santos et al., "Inhibition of local effects induced by Bothrops erythromelas snake venom: assessment of the effectiveness of Brazilian polyvalent bothropic antivenom and aqueous leaf extract of Jatropha gossypiifolia," Toxicon, vol. 125, pp. 74-83, 2017.

[120] C. A. H. Fernandes, F. F. Cardoso, W. G. L. Cavalcante et al., "Structural basis for the inhibition of a phospholipase $\mathrm{A}_{2}$-like toxin by caffeic and aristolochic acids," PLoS ONE, vol. 10, no. 7, Article ID e0133370, 2015.

[121] V. Srinivasa, M. S. Sundaram, S. Anusha et al., "Novel apigenin based small molecule that targets snake venom metalloproteases," PLoS ONE, vol. 9, no. 9, Article ID e106364, 2014.

[122] J. A. Pereañez, A. C. Patiño, V. Núñez, and E. Osorio, "The biflavonoid morelloflavone inhibits the enzymatic and biological activities of a snake venom phospholipase A2," Chemico-Biological Interactions, vol. 220, pp. 94-101, 2014.

[123] M. Molander, D. Staerk, H. Mørck Nielsen et al., "Investigation of skin permeation, ex vivo inhibition of venom-induced tissue destruction, and wound healing of African plants used against snakebites," Journal of Ethnopharmacology, vol. 165, pp. 1-8, 2015.

[124] R. Otero, V. Núñez, J. Barona et al., "Snakebites and ethnobotany in the northwest region of Colombia-Part III: Neutralization of the haemorrhagic effect of Bothrops atrox venom," Journal of Ethnopharmacology, vol. 73, no. 1-2, pp. 233241, 2000.

[125] R. Biondo, A. M. S. Pereira, S. Marcussi, P. S. Pereira, S. C. França, and A. M. Soares, "Inhibition of enzymatic and pharmacological activities of some snake venoms and toxins by Mandevilla velutina (Apocynaceae) aqueous extract," Biochimie, vol. 85, no. 10, pp. 1017-1025, 2003.

[126] E. L. G. Veronese, L. E. Esmeraldino, A. P. F. Trombone et al., "Inhibition of the myotoxic activity of Bothrops jararacussu venom and its two major myotoxins, BthTX-I and BthTX-II, by the aqueous extract of Tabernaemontana catharinensis A. DC. (Apocynaceae)," Phytomedicine, vol. 12, no. 1-2, pp. 123-130, 2005.

[127] V. Núñez, R. Otero, J. Barona et al., "Neutralization of the edema-forming, defibrinating and coagulant effects of Bothrops asper venom by extracts of plants used by healers in Columbia," Brazilian Journal of Medical and Biological Research, vol. 37, no. 7, pp. 969-977, 2004.

[128] V. M. de Moura, L. A. F. de Sousa, R. B. de Oliveira et al., "Inhibition of the principal enzymatic and biological effects of the crude venom of Bothrops atrox by plant extracts," Journal of Medicinal Plants Research, vol. 7, no. 31, pp. 2330-2337, 2013.

[129] B. Badilla, F. Chaves, G. Mora, and L. J. Poveda, "Edema induced by Bothrops asper (Squamata: Viperidae) snake venom and its inhibition by Costa Rican plant extracts," Revista de Biologia Tropical, vol. 54, no. 2, pp. 245-252, 2006.

[130] P. A. Melo, M. C. D. Nascimento, W. B. Mors, and G. SuarezKurtz, "Inhibition of the myotoxic and hemorrhagic activities of crotalid venoms by Eclipta prostrata (Asteraceae) extracts and constituents," Toxicon, vol. 32, no. 5, pp. 595-603, 1994. 
[131] V. B. Mourao, G. M. Giraldi, and L. M. G. Neves, "Anti-hemorrhagic effect of hydro-alcoholic extract of the leaves of Mikania glomerata in lesions induced by Bothrops jararaca venom in rats," Acta Cirurgica Brasileira, vol. 29, no. 1, pp. 30-37, 2014.

[132] Y. P. Motta, M. Sakate, R. M. B. Nogueira et al., "Quantification of cytokines in serum and paw homogenate of experimental intoxication for venom of the Bothropoides jararaca in Wistar rats treated with antivenom and Mikania glomerata," Arquivo Brasileiro de Medicina Veterinaria e Zootecnia, vol. 66, no. 5, pp. 1413-1418, 2014.

[133] D. P. C. Oliveira, M. R. L. Borrás, L. C. D. L. Ferreira, and J. L. López-Lozano, "Atividade antiinflamatória do extrato aquoso de Arrabidaea chica (Humb. \& Bonpl.) B. Verl. sobre o edema induzido por venenos de serpentes amazônicas," Revista Brasileira de Farmacognosia, vol. 19, no. 2B, pp. 643-49, 2009.

[134] R. Otero, V. Núñez, S. L. Jiménez et al., "Snakebites and ethnobotany in the northwest region of Colombia. Part II: Neutralization of lethal and enzymatic effects of Bothrops atrox venom," Journal of Ethnopharmacology, vol. 71, no. 3, pp. 505$511,2000$.

[135] F. K. Ticli, L. I. S. Hage, R. S. Cambraia et al., "Rosmarinic acid, a new snake venom phospholipase A2 inhibitor from Cordia verbenacea (Boraginaceae): Antiserum action potentiation and molecular interaction," Toxicon, vol. 46, no. 3, pp. 318-327, 2005.

[136] E. C. De Oliveira, M. C. Anholeti, T. F. Domingos et al., "Inhibitory effect of the plant Clusia fluminensis against biological activities of Bothrops jararaca snake venom," Natural Product Communications, vol. 9, no. 1, pp. 21-25, 2014.

[137] L. C. D. S. Picanço, J. A. H. M. Bittencourt, S. V. C. Henriques et al., "Pharmacological activity of Costus spicatus in experimental Bothrops atrox envenomation," Pharmaceutical Biology, vol. 54, no. 10, pp. 2103-2110, 2016.

[138] J. M. Fernandes, J. Félix-Silva, L. M. Da Cunha et al., "Inhibitory effects of hydroethanolic leaf extracts of kalanchoe brasiliensis and kalanchoepinnata (crassulaceae) against local effects induced by bothropsjararaca snake venom," PLOS ONE, vol. 11, no. 12, Article ID e0168658, 2016.

[139] C. M. Nishijima, C. M. Rodrigues, M. A. Silva, M. LopesFerreira, W. Vilegas, and C. A. Hiruma-Lima, "Anti-hemorrhagic activity of four brazilian vegetable species against Bothrops jararaca venom," Molecules, vol. 14, no. 3, pp. 1072-1080, 2009.

[140] L. E. Esmeraldino, A. M. Souza, and S. V. Sampaio, "Evaluation of the effect of aqueous extract of Croton urucurana Baillon (Euphorbiaceae) on the hemorrhagic activity induced by the venom of Bothrops jararaca, using new techniques to quantify hemorrhagic activity in rat skin," Phytomedicine, vol. 12 , no. 8 , pp. 570-576, 2005.

[141] J. A. D. S. Gomes, J. Félix-Silva, J. Morais Fernandes et al., "Aqueous Leaf Extract of Jatropha mollissima (Pohl) Bail Decreases Local Effects Induced by Bothropic Venom," BioMed Research International, vol. 2016, Article ID 6101742, 13 pages, 2016.

[142] V. L. Mack-Wen, L. B. Rico, J. C. Alarc, J. A. Perea, and J. C. Alarcón, "Inhibición in vitro del veneno de Bothrops asper con extractos etanólicos de Brownea ariza B. (Caesalpiniaceae)," Vitae, vol. 18, no. 1, pp. 43-48, 2011.

[143] M. Salazar, L. Chérigo, H. Acosta, R. Otero, and S. MartínezLuis, "Evaluation of anti-Bothrops asper venom activity of ethanolic extract of Brownea rosademonte leaves," Acta Pharmaceutica, vol. 64, no. 4, pp. 475-483, 2014.
[144] V. S. Nazato, L. Rubem-Mauro, N. A. G. Vieira et al., "In vitro antiophidian properties of Dipteryx alata Vogel bark extracts," Molecules, vol. 15, no. 9, pp. 5956-5970, 2010.

[145] J. O. Da Silva, J. S. Coppede, V. C. Fernandes et al., "Antihemorrhagic, antinucleolytic and other antiophidian properties of the aqueous extract from Pentaclethra macroloba, Journal of Ethnopharmacology, vol. 100, no. 1-2, pp. 145-152, 2005.

[146] L. H. F. Vale, M. M. Mendes, A. Hamaguchi, A. M. Soares, V. M. Rodrigues, and M. I. Homsi-Brandeburgo, "Neutralization of pharmacological and toxic activities of Bothrops snake venoms by Schizolobium parahyba (Fabaceae) aqueous extract and its fractions," Basic and Clinical Pharmacology and Toxicology, vol. 103, no. 1, pp. 104-107, 2008.

[147] M. M. Mendes, C. F. Oliveira, D. S. Lopes et al., "Anti-snake venom properties of Schizolobium parahyba (caesalpinoideae) aqueous leaves extract," Phytotherapy Research, vol. 22, no. 7, pp. 859-866, 2008.

[148] J. A. Perea, S. L. Jimenez, J. C. Quintana, and J. A. Pereañez, "Inhibición de las actividades proteolítica, coagulante y hemolítica indirecta inducidas por el veneno de Bothrops asper por extractos etanólicos de tres especies de heliconias," Vitae, vol. 15, no. 1, pp. 157-164, 2008.

[149] M. Assafim, E. C. Coriolano, and S. E. Benedito, "Hypericum brasiliense plant extract neutralizes some biological effects of Bothrops jararaca snake venom," Journal of Venom Research, vol. 2, pp. 11-16, 2011.

[150] H. N. Costa, M. C. Santos, A. F. Alcântara, M. C. Silva, R. C. França, and D. Piló-Veloso, "Constituintes químicos e atividade antiedematogênica de Peltodon radicans (Lamiaceae)," Química Nova, vol. 31, no. 4, pp. 744-750, 2008.

[151] M. M. Mendes, S. A. P. B. Vieira, M. S. R. Gomes et al., "Triacontyl p-coumarate: an inhibitor of snake venom metalloproteinases," Phytochemistry, vol. 86, pp. 72-82, 2013.

[152] V. M. de Moura, L. Y. de Souza, N. da Costa Guimarães et al., "The potential of aqueous extracts of Bellucia dichotoma Cogn. (Melastomataceae) to inhibit the biological activities of Bothrops atrox venom: A comparison of specimens collected in the states of Pará and Amazonas, Brazil," Journal of Ethnopharmacology, vol. 196, pp. 168-177, 2017.

[153] B. Badilla, F. Chaves, S. Jiménez, G. Rodríguez, and L. J. Poveda, "Effects of an extract of Cissampelos pareira on the hemorrhagic and proteolytic activities from Bothrops asper venom," Pharmacognosy Magazine, vol. 4, no. 13, pp. 27-31, 2008.

[154] J. A. H. M. Bittencourt, N. K. S. de Oliveira, M. S. Cabral et al., "Antiophidian activity of Brosimum guianense (AUBL) Huber," American Journal of Pharmacology and Toxicology, vol. 9, no. 2, pp. 148-156, 2014.

[155] M. H. Borges, D. L. F. Alves, D. S. Raslan et al., "Neutralizing properties of Musa paradisiaca L. (Musaceae) juice on phospholipase $\mathrm{A}_{2}$, myotoxic, hemorrhagic and lethal activities of crotalidae venoms," Journal of Ethnopharmacology, vol. 98, no. 1-2, pp. 21-29, 2005.

[156] L. A. F. Sousa, V. M. Moura, and J. D. A. Raposo, "The effect of the aqueous extract of Myrcia guianensis (AubL) DC and its fractions against the hemorrhagic activity of Bothrops jararaca venom," Journal of Medicinal Plants Research, vol. 7, no. 42, pp. 3139-46, 2013.

[157] F. G. Freitas, T. A. Silva, and F. Oliveira, "Toxicidade aguda e propriedades antiofídicas do extrato aquoso de Casearia grandiflora (Flacourtiaceae): atividades fosfolipásica $\mathrm{A}_{2}$, miotóxica e letal de peçonhas de B. moojeni e B. neuwiedi," Bioscience Journal, vol. 21, no. 2, pp. 95-103, 2005. 
[158] M. Cintra-Francischinelli, M. G. Silva, N. Andréo-Filho et al., "Antibothropic action of casearia sylvestris Sw. (flacourtiaceae) extracts," Phytotherapy Research, vol. 22, no. 6, pp. 784-790, 2008.

[159] M. H. Borges, A. M. Soares, V. M. Rodrigues et al., "Neutralization of proteases from Bothrops snake venoms by the aqueous extract from Casearia sylvestris (Flacourtiaceae)," Toxicon, vol. 39, no. 12, pp. 1863-1869, 2001.

[160] S. L. Da Silva, A. K. Calgarotto, J. S. Chaar, and S. Marangoni, "Isolation and characterization of ellagic acid derivatives isolated from Casearia sylvestris SW aqueous extract with antiPLA2 activity," Toxicon, vol. 52, no. 6, pp. 655-666, 2008.

[161] M. L. Da Silva, S. Marcussi, R. S. Fernandes et al., "Anti-snake venom activities of extracts and fractions from callus cultures of Sapindus saponaria," Pharmaceutical Biology, vol. 50, no. 3, pp. 366-375, 2012.

[162] R. S. Fernandes, T. R. Costa, S. Marcussi et al., "Neutralization of pharmacological and toxic activities of Bothrops jararacussu snake venom and isolated myotoxins by Serjania erecta methanolic extract and its fractions," Journal of Venomous Animals and Toxins Including Tropical Diseases, vol. 17, no. 1, pp. 85-93, 2011.

[163] N. Tribuiani, A. M. da Silva, M. C. Ferraz et al., "Vellozia flavicans Mart. ex Schult. hydroalcoholic extract inhibits the neuromuscular blockade induced by Bothrops jararacussu venom," BMC Complementary and Alternative Medicine, vol. 14, article 48, 2014.

[164] M. M. Melo, M. Lúcia, and G. G. Habermehl, "Plant extracts for topic therapy of Bothrops alternatus envenomation," Brazilian Journal of Pharmacognosy, vol. 17, no. 1, pp. 29-34, 2007.

[165] M. Fernández, W. F. Ortiz, J. A. Pereáñez, and D. Martínez, "Evaluación de las propiedades antiofídicas del extracto etanólico y fracciones obtenidas de Renealmia alpinia(Rottb) Mass (Zingiberaceae) cultivada in vitro," Vitae, vol. 17, no. 1, pp. 75-82, 2010.

[166] A. C. Patiño, J. López, M. Aristizábal, J. C. Quintana, and D. Benjumea, "Evaluation of the inhibitory effect of extracts from leaves of renealmia alpinia rottb. maas(Zingiberaceae) on the venom of bothrops asper (mapaná)," Biomedica, vol. 32, no. 3 , pp. 365-374, 2012.

[167] O. J. Ode and I. U. Asuzu, "The anti-snake venom activities of the methanolic extract of the bulb of Crinum jagus (Amaryllidaceae)," Toxicon, vol. 48, no. 3, pp. 331-342, 2006.

[168] J. M. Gutiérrez, A. Rucavado, F. Chaves, C. Díaz, and T. Escalante, "Experimental pathology of local tissue damage induced by Bothrops asper snake venom," Toxicon, vol. 54, no. 7, pp. 958975, 2009.

[169] D. Paixão-Cavalcante, A. K. Kuniyoshi, F. C. V. Portaro, W. D. da Silva, and D. V. Tambourgi, "African Adders: Partial Characterization of Snake Venoms from Three Bitis Species of Medical Importance and Their Neutralization by Experimental Equine Antivenoms," PLoS Neglected Tropical Diseases, vol. 9, no. 2, Article ID e0003419, 2015.

[170] S. Ushanandini, S. Nagaraju, S. C. Nayaka, K. H. Kumar, K. Kemparaju, and K. S. Girish, "The anti-ophidian properties of Anacardium occidentale bark extract," Immunopharmacology and Immunotoxicology, vol. 31, no. 4, pp. 607-615, 2009.

[171] B. L. Dhananjaya, F. Zameer, K. S. Girish, and C. J. M. D’Souza, "Anti-venom potential of aqueous extract of stem bark of Mangifera indica L. against Daboia russellii (Russell's viper) venom," Indian Journal of Biochemistry and Biophysics, vol. 48, no. 3, pp. 175-183, 2011.
[172] M. I. Alam, B. Auddy, and A. Gomes, "Viper venom neutralization by Indian medicinal plant (Hemidesmus indicus and Pluchea indica) root extracts," Phytotherapy Research, vol. 10, no. 1, pp. 58-61, 1996.

[173] P. Bhattacharjee and D. Bhattacharyya, "Characterization of the aqueous extract of the root of Aristolochia indica: evaluation of its traditional use as an antidote for snake bites," Journal of Ethnopharmacology, vol. 145, no. 1, pp. 220-226, 2013.

[174] A. Shirwaikar, K. Rajendran, R. Bodla, and C. D. Kumar, "Neutralization potential of Viper russelli russelli (Russell's viper) venom by ethanol leaf extract of Acalypha indica," Journal of Ethnopharmacology, vol. 94, no. 2-3, pp. 267-273, 2004.

[175] S. Tarannum, R. Mohamed, and B. S. Vishwanath, "Inhibition of testicular and Vipera russelli snake venom hyaluronidase activity by Butea monosperma (Lam) Kuntze stem bark," Natural Product Research, vol. 26, no. 18, pp. 1708-1711, 2012.

[176] K. M. Maung and Z. Lynn, "Effects of Tamarind (Tamarindus indicus Linn) seed extract on Russell's viper (Daboia russelli siamensis) venom," Tropical Biomedicine, vol. 29, no. 4, pp. 580$587,2012$.

[177] S. Ushanandini, S. Nagaraju, K. H. Kumar et al., “The antisnake venom properties of Tamarindus indica (leguminosae) seed extract," Phytotherapy Research, vol. 20, no. 10, pp. 851-858, 2006.

[178] M. I. Alam and A. Gomes, "Snake venom neutralization by Indian medicinal plants (Vitex negundo and Emblica officinalis) root extracts," Journal of Ethnopharmacology, vol. 86, no. 1, pp. 75-80, 2003.

[179] K. T. Chandrashekara, S. Nagaraju, S. Usha Nandini, Basavaiah, and K. Kemparaju, "Neutralization of local and systemic toxicity of Daboia russelii venom by Morus alba plant leaf extract," Phytotherapy Research, vol. 23, no. 8, pp. 1082-1087, 2009.

[180] S. A. Krishnan, R. Dileepkumar, A. S. Nair, and O. V. Oommen, "Studies on neutralizing effect of Ophiorrhiza mungos root extract against Daboia russelii venom," Journal of Ethnopharmacology, vol. 151, no. 1, pp. 543-547, 2014.

[181] B. Janardhan, V. M. Shrikanth, K. K. Mirajkar, and S. S. More, "In vitro screening and evaluation of antivenom phytochemicals from Azima tetracantha Lam. leaves against bungarus caeruleus and Vipera russelli," Journal of Venomous Animals and Toxins Including Tropical Diseases, vol. 20, no. 1, article 12, 2014.

[182] Y. H. Mahadeswaraswamy, S. Devaraja, M. S. Kumar, Y. N. J. Goutham, and K. Kemparaju, "Inhibition of local effects of Indian Daboia/Vipera russelli venom by the methanolic extract of grape (Vitis vinifera L.) seeds," Indian Journal of Biochemistry and Biophysics, vol. 46, no. 2, pp. 154-160, 2009.

[183] A. K. Mukherjee, B. Kalita, and S. P. Mackessy, "A proteomic analysis of Pakistan Daboia russelii russelii venom and assessment of potency of Indian polyvalent and monovalent antivenom," Journal of Proteomics, vol. 144, pp. 73-86, 2016.

[184] W. Wüster, “The genus Daboia (Serpentes: Viperidae): Russell's viper," Hamadryad, vol. 23, no. 1, pp. 33-40, 1998.

[185] D. A. Warrell, “Snake bite," The Lancet, vol. 375, no. 9708, pp. 77-88, 2010.

[186] D. Pla, L. Sanz, P. Molina-Sánchez et al., "Snake venomics of Lachesis muta rhombeata and genus-wide antivenomics assessment of the paraspecific immunoreactivity of two antivenoms evidence the high compositional and immunological conservation across Lachesis," Journal of Proteomics, vol. 89, pp. 112-123, 2013. 
[187] J. M. Gutiérrez, "Comprendiendo los venenos de serpientes: 50 Años de investigaciones en América Latina," Revista de Biologia Tropical, vol. 50, no. 2, pp. 377-394, 2002.

[188] R. C. de Paula, E. F. Sanchez, T. R. Costa et al., "Antiophidian properties of plant extracts against Lachesis muta venom," Journal of Venomous Animals and Toxins Including Tropical Diseases, vol. 16, no. 2, pp. 311-323, 2010.

[189] E. C. De Oliveira, R. A. S. Cruz, N. D. M. Amorim et al., "Protective effect of the plant extracts of erythroxylum sp. against toxic effects induced by the venom of lachesis muta snake," Molecules, vol. 21, no. 10, article 1350, 2016.

[190] R. Biondo, A. M. Soares, B. W. Bertoni, S. C. França, and A. M. S. Pereira, "Direct organogenesis of Mandevilla illustris (Vell) Woodson and effects of its aqueous extract on the enzymatic and toxic activities of Crotalus durissus terrificus snake venom," Plant Cell Reports, vol. 22, no. 8, pp. 549-552, 2004.

[191] O. J. Ode, C. O. Nwaehujor, and M. M. Onakpa, "Evaluation of antihaemorrhagic and antioxidant potentials of Crinum jagus bulb," International Journal of Applied Biology and Pharmaceutical Technology, vol. 1, no. 3, pp. 1330-1336, 2010.

[192] A. Nalbantsoy, Ş. B. Erel, Ç. Köksal, B. Göçmen, M. Z. Yildiz, and N. Ü. Karabay Yavaşoĝlu, "Viper venom induced inflammation with Montivipera xanthina (Gray, 1849) and the anti-snake venom activities of Artemisia absinthium L. in rat," Toxicon, vol. 65, pp. 34-40, 2013.

[193] R. D. C. O. Collaço, J. C. Cogo, L. Rodrigues-Simioni, T. Rocha, Y. Oshima-Franco, and P. Randazzo-Moura, "Protection by Mikania laevigata (guaco) extract against the toxicity of Philodryas olfersii snake venom," Toxicon, vol. 60, no. 4, pp. 614-622, 2012.

[194] P. U. Amog, V. N. Manjuprasanna, M. Yariswamy et al., "Albizia lebbeck seed methanolic extract as a complementary therapy to manage local toxicity of Echis carinatus venom in a murine model," Pharmaceutical Biology, vol. 54, no. 11, pp. 2568-2574, 2016.

[195] A. N. Nanjaraj Urs, M. Yariswamy, V. Joshi et al., "Local and systemic toxicity of Echis carinatus venom: Neutralization by Cassia auriculata L. leaf methanol extract," Journal of Natural Medicines, vol. 69, no. 1, pp. 111-122, 2014.

[196] S. S. Hasson, M. S. Al-Balushi, E. A. Said et al., "Neutralisation of local haemorrhage induced by the saw-scaled viper Echis carinatus sochureki venom using ethanolic extract of Hibiscus aethiopicus L," Evidence-Based Complementary and Alternative Medicine, vol. 2012, Article ID 540671, 8 pages, 2012.

[197] S. S. Hasson, A. A. Al-Jabri, T. A. Sallam, M. S. Al-Balushi, and R. A. A. Mothana, "Antisnake venom activity of hibiscus aethiopicus L. against echis ocellatus and naja n. nigricollis," Journal of Toxicology, vol. 2010, Article ID 837864, 2010.

[198] Y. H. Mahadeswaraswamy, S. Nagaraju, K. S. Girish, and K. Kemparaju, "Local tissue destruction and procoagulation properties of Echis carinatus venom: Inhibition by Vitis vinifera seed methanol extract," Phytotherapy Research, vol. 22, no. 7, pp. 963-969, 2008.

[199] J. Boldrini-França, C. Corrêa-Netto, M. M. S. Silva et al., "Snake venomics and antivenomics of Crotalus durissus subspecies from Brazil: Assessment of geographic variation and its implication on snakebite management," Journal of Proteomics, vol. 73, no. 9, pp. 1758-1776, 2010.

[200] M. L. Santoro, M. C. C. Sousa-E-Silva, L. R. C. Gonçalves et al., "Comparison of the biological activities in venoms from three subspecies of the South American rattlesnake (Crotalus durissus terrificus, C. durissus cascavella and C. durissus collilineatus)," Comparative Biochemistry and Physiology - C Pharmacology Toxicology and Endocrinology, vol. 122, no. 1, pp. 61-73, 1999.

[201] F. Sangiorgio, M. Sakate, R. M. B. Nogueira, J. P. Araújo Jr., and C. Chavez-Olortegui, "Kinetics of venom and antivenom serum levels, clinical evaluation and therapeutic effectiveness in dogs inoculated with Crotalus durissus terrificus venom," Journal of Venomous Animals and Toxins Including Tropical Diseases, vol. 14, no. 1, pp. 100-112, 2008.

[202] V. Hiremath, A. N. Nanjaraj Urs, V. Joshi et al., "Differential action of medically important Indian BIG FOUR snake venoms on rodent blood coagulation," Toxicon, vol. 110, pp. 19-26, 2016.

[203] Q. Chen, W. Wang, Q. Li, Y. Bai, X. Zou, and Y. Wu, "Effect of externally applied Jidesheng anti-venom on skin and soft-tissue necrosis after Chinese cobra bite: a retrospective study," Journal of Traditional Chinese Medicine, vol. 34, no. 2, pp. 150-154, 2014. 


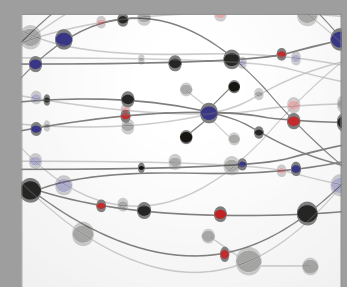

The Scientific World Journal
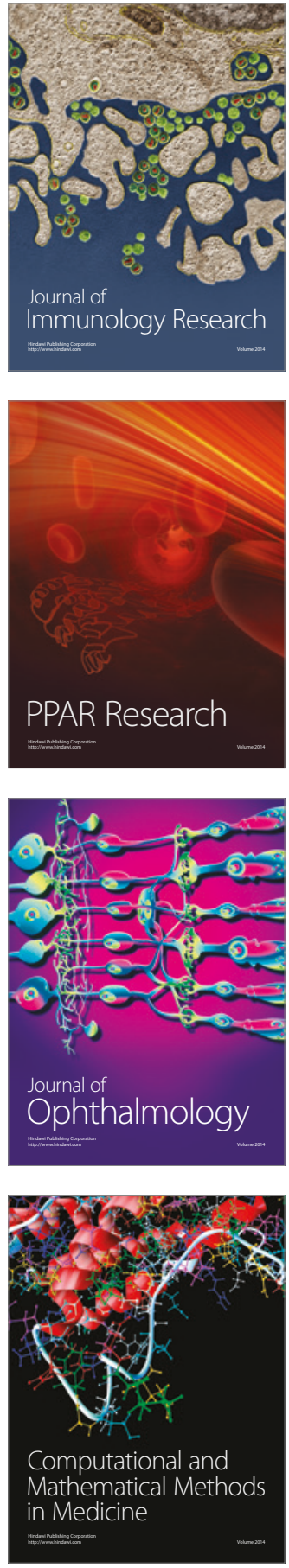

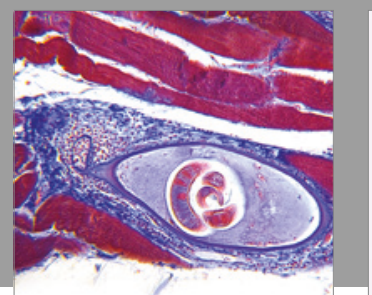

Gastroenterology Research and Practice
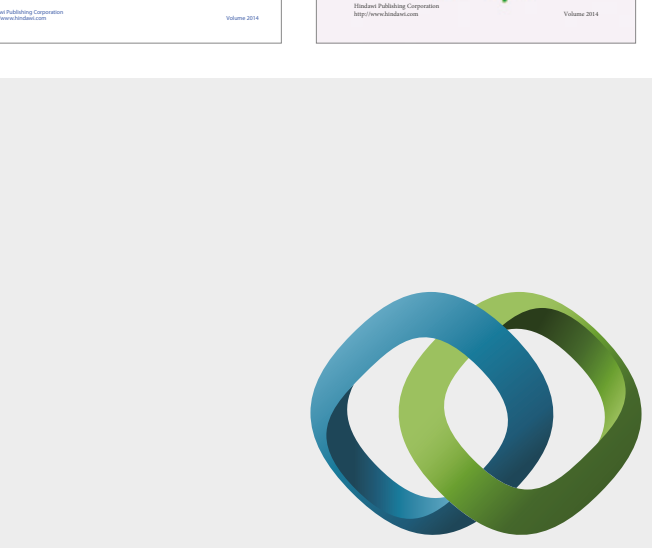

\section{Hindawi}

Submit your manuscripts at

https://www.hindawi.com
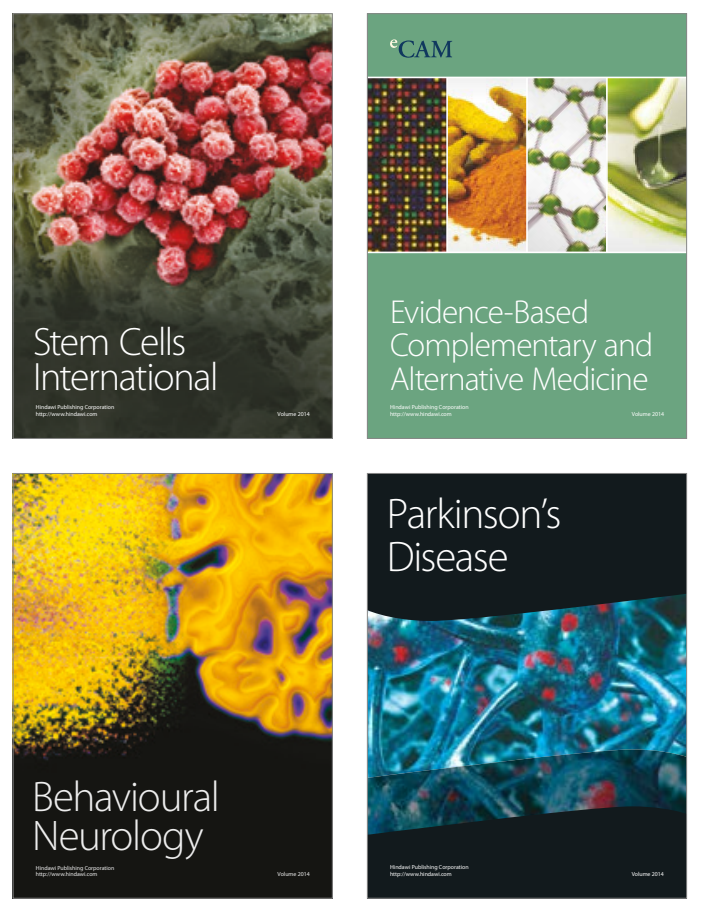
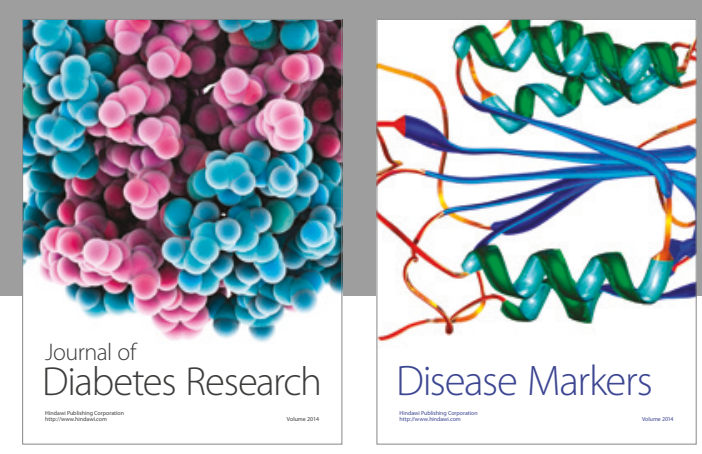

Disease Markers
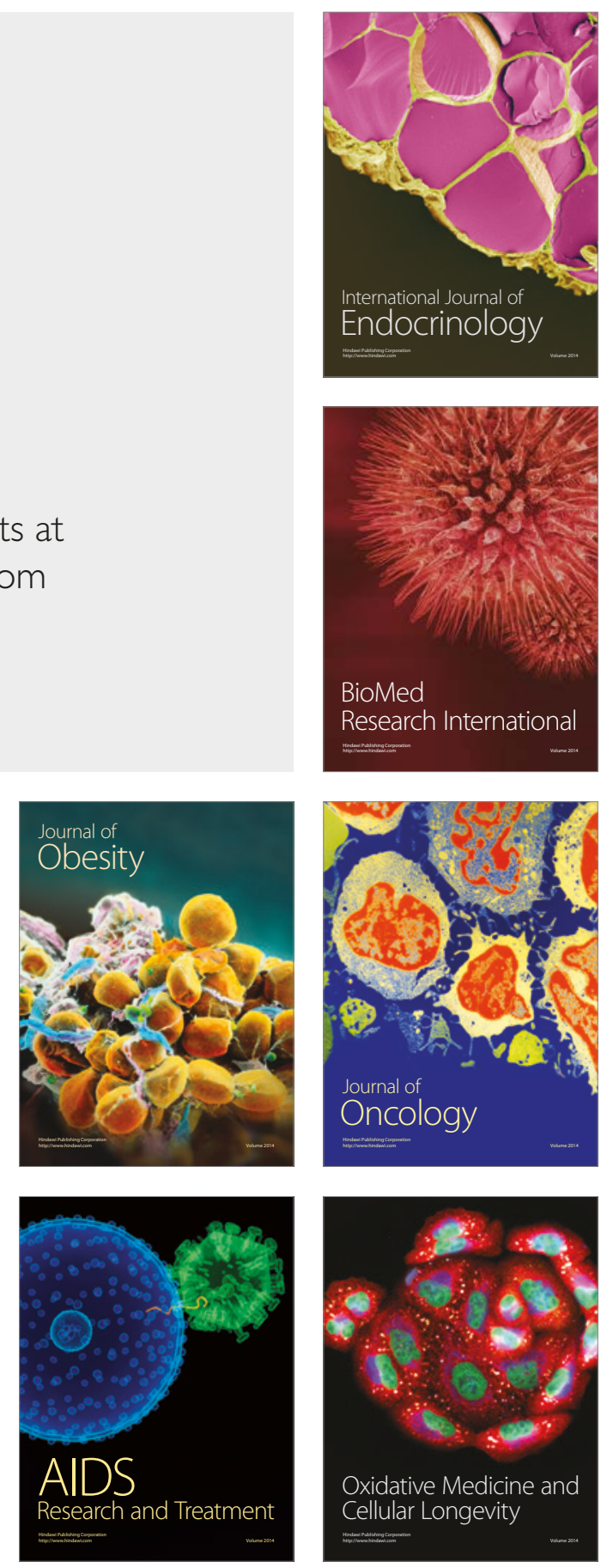